\title{
A hyper-Kähler compactification of the intermediate Jacobian fibration associated with a cubic 4-fold
}

by

RADU LAZA

Stony Brook University Stony Brook, NY, U.S.A.

\section{Givlia SACCÀ}

Stony Brook University Stony Brook, NY, U.S.A.

\author{
Claire Voisin \\ Collège de France \\ Paris, France
}

\section{Introduction}

It is a problem of significant interest to construct and classify compact hyper-Kähler (HK) manifolds. In dimension 2, the HK manifolds are K3 surfaces. All known higher dimensional examples are obtained from K3s or abelian varieties, by a moduli construction and a deformation. Specifically, Beauville [9] has given two series of examples: the Hilbert scheme of points on K3s and generalized Kummer varieties, respectively. Mukai [47] has given a more general construction, namely he has shown that the moduli space of semi-stable sheaves on K3s carries a symplectic form, and thus is a HK manifold if it is smooth. Unfortunately, these examples are deformation equivalent to those of Beauville [67]. Starting from a singular moduli space of sheaves on K3s, O'Grady [50], [51] has produced two genuinely new examples: a 10-dimensional and a 6-dimensional one, that we call OG10 and OG6, respectively. It was subsequently verified ([38]) that these are the only two new examples that can be obtained by this method.

It is natural to expect that hyper-Kähler manifolds can be constructed from lowerdimensional objects of similar nature. Specifically, we recall that the moduli space of polarized HK manifolds in a fixed deformation class is birational via the period map to a locally symmetric variety $\mathscr{D} / \Gamma$, where $\mathscr{D}$ is a type-IV domain, and $\Gamma$ an arithmetic group. Such a locally symmetric variety contains divisors $\mathscr{D}^{\prime} / \Gamma^{\prime}$ (known as NoetherLefschetz or Heegner divisors), which are of the same type. It is natural to expect that 
some of these NL divisors are associated (at least motivically) with moduli spaces of lower-dimensional HK manifolds. For instance, the Beauville-Mukai construction will give such an example (i.e. Hilb ${ }^{n}(\mathrm{~K} 3)$ form an NL divisor in the corresponding moduli space of $2 n$-dimensional HK manifolds). Another NL divisor in the moduli space, that we will call a Lagrangian NL divisor, is obtained by considering the HK manifolds that admit a Lagrangian fibration. Again, one can hope that they can be constructed from lower-dimensional geometric objects.

As polarized K3 surfaces have only 19 parameters, Hodge theory and abstract deformation theory show that many hyper-Kähler varieties built from polarized K3 surfaces have projective deformations which are unrelated to K3 surfaces. However, the problem of constructing explicit projective models for these deformations is usually hard, one reason being the fact that most of these deformation spaces are of general type ([28]). Cubic 4-folds have 20 moduli and they are well known to have a Hodge structure with Hodge numbers $h^{3,1}=1, h_{\text {prim }}^{2,2}=20$. In several instances, cubic 4 -folds have been used to provide via an auxiliary construction such an algebro-geometric deformation of Hilbert schemes of K3 surfaces. For instance [13], one of the key papers in the field, shows that the Fano variety of lines of a cubic $X$ is a deformation of a $\operatorname{Hilb}^{2}(\mathrm{~K} 3)$. More recently, [42] constructs a HK manifold from the variety of cubic rational curves in $X$, which is then shown in [1] to be deformation equivalent to a $\operatorname{Hilb}^{4}(\mathrm{~K} 3)$.

The 10-dimensional examples by O'Grady have $b_{2}=24$, which means that polarized deformations of them have 21 moduli, and complete families of such varieties with Picard number 2 have 20 moduli. The construction by O'Grady in [50] provides (infinitely many) 19-parameters families of such examples with Picard number 3, parameterized by any moduli space of polarized K3 surfaces. The present paper provides an algebrogeometric realization of the moduli space of cubic 4-folds as a 20-dimensional moduli space of deformations of O'Grady's 10-dimensional examples, and more precisely, as a Lagrangian NL divisor in the larger 21-dimensional deformation space (our varieties are canonically lattice polarized). Note first that this embedding is a priori given by lattice considerations. Indeed, the moduli space of cubic 4-folds is birational to a 20-dimensional locally symmetric variety $\mathscr{D}^{\prime} / \Gamma^{\prime}$, which is associated with the lattice $A_{2} \oplus E_{8}^{2} \oplus U^{2}$ (where $A_{2}$ and $E_{8}$ are the standard positive definite root lattices, and $U$ is the hyperbolic plane). On the other hand, by work of Rapagnetta [55], it is known that the second cohomology of OG10 equipped with the Beauville-Bogomolov form is isometric to the lattice

$$
A_{2}(-1) \oplus E_{8}(-1)^{2} \oplus U^{3} .
$$

This shows that, from an arithmetic point of view, the situation is similar to that of elliptic K3s with a section (lattice $E_{8}(-1)^{2} \oplus U^{2}$ ), also known as unigonal K3s, versus 
general K3s (lattice $\left.E_{8}(-1)^{2} \oplus U^{3}\right)$. Furthermore, it is not hard to embed the period space of cubic 4-folds as a NL divisor into a 21-dimensional period domain of polarized OG10 manifolds (for K3s, the unigonal K3s form a Heegner divisor in any of the polarized period domains).

Our contribution in this paper is to realize geometrically this abstract embedding by actually constructing a family of OG10 polarized manifolds parameterized by the moduli space of cubic 4-folds. This is done by realizing the following program that has been started by Donagi-Markman and developed by Markushevich, with further evidence provided by work of Kuznetsov and O'Grady-Rapagnetta: Starting from a general cubic 4-fold $X$, one has the universal family $\mathcal{Y} / B$ of cubic 3 -folds over $B:=\left(\mathbb{P}^{5}\right)^{\vee}$ obtained as hyperplane sections of $X$, and then the associated relative intermediate Jacobian fibration $\mathcal{J}_{U} / U$, where $U=B \backslash X^{\vee} \subset B$ is the locus of smooth hyperplane sections. In 1993, Donagi and Markman [22] had the insight that this fibration, which they showed to be algebraic, carries a holomorphic symplectic form. The question naturally was raised, as to whether or not it admitted a holomorphic symplectic compactification. If such an algebraic or Kähler holomorphic symplectic compactification $\overline{\mathcal{J}} / B$ exists, then it has Picard number at least 2 and transcendental second cohomology containing the transcendental cohomology of the cubic $X$. Thus, $b_{2}(\overline{\mathcal{J}}) \geqslant 24$, showing in particular that $\overline{\mathcal{J}}$ cannot be deformation equivalent to $\mathrm{K}^{[5]}$ type, but potentially equivalent to OG10. Markushevich and Kuznetsov [40] (with further supporting evidence by O'Grady and others) conjectured that indeed a good compactification $\overline{\mathcal{J}}$ exists, and that it is of OG10 type. In this paper, we verify this conjecture. Specifically, we prove the following.

MAin THEOREM (Cf. Theorem 5.7 and Corollary 6.3.) Let $X \subset \mathbb{P}^{5}$ be a general cubic 4-fold. Let $B=\left(\mathbb{P}^{5}\right)^{\vee}, U=\left(B \backslash X^{\vee}\right)$, and $\mathcal{J}_{U} \rightarrow U$ be the associated intermediate Jacobian fibration. Then there exists a smooth projective compactification $\overline{\mathcal{J}} \rightarrow B$, which carries a holomorphic symplectic form, with respect to which the fibration is Lagrangian. Furthermore, $\overline{\mathcal{J}}$ is deformation equivalent to OG10.

The key issue for the theorem, and the main new content of the paper, is the construction of a smooth projective compactification of the intermediate Jacobian fibration that has a non-degenerate holomorphic 2-form. In order to do so, it is important to understand degenerations of intermediate Jacobians as the hyperplane section of $X$ becomes singular. The study of intermediate Jacobians from a different perspective was done in [17], [16], and the main tool used there is Mumford's construction of the intermediate Jacobian as a Prym variety. This is our approach also here. A key point that allows us to construct the compactification $\overline{\mathcal{J}} / B$ is the observation that much of the Prym construction goes through for mildly singular cubic 3-folds. Namely, we recall that 
for a smooth cubic 3-fold $Y$, the projection from a generic line $l$ realizes $Y$ as a conic bundle over $\mathbb{P}^{2}$ with discriminant locus a smooth quintic $C$. Then, Mumford showed that $J(Y) \cong \operatorname{Prym}(\widetilde{C} / C)$, where $\widetilde{C}$ is an étale double cover of $C$. In [17], the authors have noted that for mildly singular cubics $Y$ there still exist good lines $l$ (see Definition 2.2) such that the associated quintic $C$ has a one-to-one correspondence with the singularities of $Y$ (including the type), and that the covering $\widetilde{C} / C$ is still étale. This reduces questions about degenerations of cubic 3 -folds to degenerations of curves. In [16], the degeneration of the Prym variety $\operatorname{Prym}(\widetilde{C} / C)$ is studied from the perspective of stable abelian varieties (in the sense of Alexeev). Here we study this from the perspective of compactified relative Jacobians (or rather compactified Pryms) instead. For curves, one has a very good understanding of the degeneration of Jacobians. Namely, from the perspective of moduli spaces, the correct statement is the theorem of Mumford-Namikawa, according to which there exists an extended period map from $\overline{\mathcal{M}}_{g}$ to $\overline{\mathcal{A}}_{g}^{\text {Vor }}$ (and thus with a family of DM stable curves one can associate a family of Alexeev stable abelian varieties). If one insists instead on associating with an arbitrary family of curves $\mathcal{C} / B$ a family of degenerate abelian varieties, without modifying the base, one gets into the theory of compactified relative Jacobians (to pass from the relative compactified Jacobian to the family of stable abelian varieties, one needs to perform a simultaneous semi-stable reduction, see [18] and the references within). The situation for Prym varieties is more complicated, but still well understood (see [24], [2], [15]) when the point of view is that of Beauville's admissible covers compactification; the relative compactified Prym is less studied but a few cases, namely when the families of curves come from linear systems on surfaces, were studied in [45], [7], and [58]. In our situation, due to the existence of very good lines, we can make an important simplifying assumption: namely, we consider only étale double covers of planar curves, and furthermore we may assume that both the cover and the base are irreducible.

Finally, we should remark that this construction also provides the first example of hyper-Kähler manifolds admitting a Lagrangian fibration in principally polarized abelian varieties that are not Jacobians of curves. Indeed, the known constructions of compact holomorphic symplectic varieties that are fibered in principally polarized abelian varieties are either the relative compactified Jacobian of a linear system on a K3 surface (Beauville-Mukai system), or the relative compactified Prym associated with a linear system on a K3 with an anti-symplectic involution ([45], [7]). In these last examples, either the relative Prym varieties are isomorphic to Jacobian of curves, in which case their total spaces are hyper-Kähler manifolds deformation equivalent to the Hilbert scheme of points on a K3 surface, or the total space of the family has singularities that admit no symplectic resolution. 
Let us briefly describe the main steps of the paper.

\section{The hyper-Kähler structure}

In $\S 1$, we review the construction of the relative intermediate Jacobian fibration $\mathcal{J}_{U} \rightarrow U$ and the existence of a holomorphic symplectic 2-form. We then show, without much difficulty, that this symplectic form extends over the locus of cubics with a single ordinary node $\mathcal{J}_{U_{1}} \rightarrow U_{1}$, providing a non-degenerate holomorphic 2-form on $\mathcal{J}_{U_{1}}$. These results are well known, and are essentially completely contained in [22, §8.5.2], but the cycletheoretic method used here has the advantage of providing an extension to a holomorphic closed 2-form on any smooth algebraic compactification $\overline{\mathcal{J}}$ of $\mathcal{J}_{U_{1}}$. If furthermore $\mathcal{J}_{U_{1}} \subset \overline{\mathcal{J}}$ has codimension larger than 2 , then the extended form is everywhere non-degenerate. This allows us to concentrate on the problem of constructing a smooth compactification of $\mathcal{J}_{U_{1}}$ which is flat over $B$. The fact that the variety we construct is irreducible holomorphic symplectic (or hyper-Kähler) makes use of [42]. Indeed, the intermediate Jacobian fibration contains a divisor which is birationally a $\mathbb{P}^{1}$-bundle on the hyper-Kähler 8 -fold recently constructed in [42].

\section{Local compactification}

In $\S 2$, we briefly recall the Prym construction, and discuss the existence of a good line for all hyperplane sections of a general cubic 4 -fold. Roughly speaking the existence of a good line guarantees that the deformation theory of cubic 3-folds and their singularities can be identified locally, up to a smooth factor, with the corresponding deformation theory of quintic curves (see $[16, \S 3]$ ). Studying the degenerations of intermediate Jacobians thus reduces to studying degenerations of abelian varieties associated with curves (more precisely Prym varieties). Some of the results and ideas in this section previously occurred in [17], [16]. Here we obtain a slight strengthening applicable to our context: for any hyperplane section $Y$ of a general cubic 4 -fold, there exists a very good line $l$, that is, a line such that the associated cover $\widetilde{C} / C$ is étale, and both curves are irreducible (with singularities in one-to-one correspondence with those of the cubic 3-fold $Y$ ). With these assumptions, there exists a (canonical) relative compactified Prym fibration, and our main result here is Theorem 4.20, which says that this compactification has the property that the total space is smooth, provided that the family of (base) curves gives a simultaneous versal deformation of the singularities (an analogue of the corresponding result for Jacobians). Moreover, this relative compactified Prym fibration is equidimensional, in particular flat over a smooth base. These results are discussed in $\S 4$. 
While $\S 4$ gives a general construction for a smooth compactification of a family of Prym varieties (under suitable assumptions), the fact that this is applicable to our situation follows from the versality statements of $\S 3$ (e.g. Corollary 3.9). It is here, in $\S 3$, that the generality assumption on the cubic 4 -fold $X$ is essential. Moreover, for the purpose of proving the deformation equivalence of our compactified fibration $\overline{\mathcal{J}} / B$ to OG10, we need to show that the versality statements still hold for general Pfaffian cubic 4-folds (§3.2).

\section{Descent}

Let $\mathscr{F} \rightarrow B$ be the relative Fano surface associated with the family $u: \mathcal{Y} \rightarrow B$ of hyperplane sections of a fixed cubic 4 -fold, and $\mathscr{F}^{0} \rightarrow B$ be the open subset of very good lines. The results of the previous three sections give that $\mathscr{F}^{0} \rightarrow B$ is a surjective smooth map (with 2-dimensional fibers), and the existence of a relative compactified Prym fibration $\overline{\mathcal{P}} \rightarrow \mathscr{F} 0$ which is proper over $\mathscr{F}^{0}$, with smooth total space $\overline{\mathcal{P}}$. Our compactification $\overline{\mathcal{J}}$ is a descent of $\overline{\mathcal{P}} \rightarrow \mathscr{F}^{0}$ to $B$. More precisely, in $\S 5$, using the relative theta divisor and a relative Proj construction, we conclude that $\overline{\mathcal{P}}$ descends to $B$, giving a smooth compactification $\overline{\mathcal{J}} \rightarrow B$ of $\mathcal{J}_{U} \rightarrow U$ extending $\mathcal{J}_{U_{1}} \rightarrow U_{1}$ and equidimensional over $B$. The arguments of $\S 1$ now allow us to conclude that $\overline{\mathcal{J}}$ is a 10-dimensional compact HK manifold, and that $\overline{\mathcal{J}}$ is a Lagrangian fibration (whose general fiber is an intermediate Jacobian). This concludes (see Theorem 5.7) the proof of the first half of the main theorem.

\section{Relationship with O'Grady's varieties}

In the final section, we establish that the constructed object is in fact deformation equivalent to the OG10 example [50] (see Corollary 6.3). Assuming the existence of a smooth compactification of $\mathcal{J}_{U}$, partial results in this direction had been established by O'GradyRapagnetta. Their idea was to use the degeneration of the cubic 4-fold to the chordal cubic (the secant variety of the Veronese surface), for which one can show that the compactification of the limiting family of intermediate Jacobians is birational to the O'Grady moduli space for an adequate K3 surface. A similar construction was provided by Hwang and Nagai [35] in the case of a singular cubic 4-fold, for which the K3 surface is the surface of lines through the singular point. Unfortunately, in both cases, we could not deduce from the existence of these birational maps the fact that our compactified Jacobian fibration is deformation equivalent to the O'Grady moduli space, because we have no control of the singularities of the compactified Jacobian fibration at these points. We study instead, as suggested also by Markushevich and Kuznetsov [40], the intermediate 
Jacobian fibration in the case of a Pfaffian cubic 4-fold. By Beauville-Donagi [13], such a cubic $X$ is Hodge-theoretic equivalent to a degree-14 K3 surface S. Using [37], [44], and [41] we show that the fibration $\mathcal{J}_{U}$ (or the compact version) is birational to the O'Grady moduli space of sheaves on $S$. On the other hand, using versality statements established in $\S 3$, we are able to prove that the compactified intermediate Jacobian fibration is smooth also for a general Pfaffian cubic 4-fold, so that Huybrechts' fundamental theorem [33] applies, allowing us to conclude the deformation equivalence to the OG10 example.

\section{Two remaining questions}

We point out that there are two families of intermediate Jacobians associated to the family of hyperplane sections of a cubic 4-fold $X$ : in addition to the $\mathcal{J}_{U} \rightarrow U$ considered here, there is a twisted family $\mathcal{J}_{U}^{\prime} \rightarrow U$ parameterizing 1-cycles of degree 1 (or 2) in the fibers of $u: \mathcal{Y}_{U} \rightarrow U$. In this paper, we are compactifying the untwisted family $\mathcal{J}_{U}$. A natural question, that will be addressed elsewhere, is the existence of a compactification for the twisted case. Here we only note that once a line has been chosen on a cubic 3 -fold, the intermediate Jacobian and the twisted intermediate Jacobian are naturally identified. Thus, up to the descent argument of $\S 5$ everything goes through unchanged.

On a related note, our construction is somewhat indirect (e.g. it involves the auxiliary choice of a line). It is natural to ask if a more direct construction is possible, in particular one wonders if there is a modular construction for our compactification $\overline{\mathcal{J}} \rightarrow B$ at least up to natural birational modifications (e.g. resolution of symplectic singularities, or contraction of some divisor on which the symplectic form is degenerate). We remind the reader that O'Grady's original construction for OG10 is indeed modular in this sense (it is the resolution of the moduli of sheaves on K3s for a specific choice of Mukai vector). Results of [12], [44] in a relative setting show that after blowing-up $\mathcal{J}_{U}$ (or rather its twisted version) along the universal family of lines, one gets a variety which is a relative moduli space of coherent sheaves supported on smooth hyperplane sections of $X$, and it is maybe possible to recontract it to a symplectic moduli space of coherent sheaves on $X$. The compactification of the present paper is birational to a moduli space of sheaves on the Fano variety of lines on $X$ (supported on the Fano surfaces of lines on the hyperplane sections of $X$ ). A possible strategy to find a modular compactification is therefore to study the smoothness of this moduli space of sheaves. 


\section{Acknowledgement}

This work was started while the authors were visiting the Institute for Advanced Studies (IAS) as part of the special program "Topology of Algebraic Varieties". We are grateful to IAS for the wonderful research environment. The IAS stay of the first author was partially supported by NSF grant DMS-1128155. He also acknowledges the support through NSF grants DMS-125481 and DMS-1361143. The second author acknowledges the support of the Giorgio and Elena Petronio Fellowship Fund II and of NSF grant DMS1128155. Claire Voisin was a distinguished visiting Professor at IAS during academic year 2014/15 and acknowledges the generous support of the Charles Simonyi Fund and of the Fernholz Foundation.

We learned about this conjecture from a talk by D. Markushevich at the HyperKähler Geometry Workshop at the Simons Center for Geometry and Physics in Fall 2012. It was he who initiated the project and subsequently and independently realized many steps towards its completion. The main result of the paper should thus partly be attributed to him. K. O'Grady and A. Rapagnetta also significantly contributed to the general project. We also acknowledge helpful discussions with E. Arbarello, S. Casalaina-Martin, K. Hulek, A. Kuznetsov, E. Macrì, L. Migliorini, K. O'Grady, and A. Rapagnetta. Finally, we are grateful to the referee for careful reading and constructive comments that helped us improve the paper.

\section{Notation and conventions}

Unless otherwise specified, $X \subset \mathbb{P}^{5}$ denotes a (Hodge) general cubic 4-fold. Let $B=\left(\mathbb{P}^{5}\right)^{\vee}$, $U=B \backslash X^{\vee}$, and let $U_{1}=B \backslash\left(X^{\vee}\right)^{\text {sing }}$ be the open set parameterizing hyperplane sections with at most one single ordinary node. Thus $U \subset U_{1} \subset B$, and $U_{1}$ has boundary of codimension 2 in $B$. Let $u: \mathcal{Y} \rightarrow B$ be the universal family of cubic 3 -folds obtained as hyperplane sections of $X$, and $u: \mathcal{Y}_{U} \rightarrow U$ and $u: \mathcal{Y}_{U_{1}} \rightarrow U_{1}$ be its restrictions to $U$ and $U_{1}$, respectively. Typically, $Y$ or $Y_{t}$ will denote hyperplane sections of $X$ or fibers of $\mathcal{Y}$. We let $\pi_{U}: \mathcal{J}_{U} \rightarrow U$ denote the intermediate Jacobian fibration associated with the family $\mathcal{Y}_{U} \rightarrow U$, and similarly for $\pi_{U_{1}}: \mathcal{J}_{U_{1}} \rightarrow U_{1}$. Thus $\mathcal{J}_{U}$ and $\mathcal{J}_{U_{1}}$ are smooth quasi-projective varieties together with a holomorphic symplectic form, and the restriction of $\mathcal{J}_{U_{1}}$ to $U$ is $\mathcal{J}_{U}$ (cf. [22] and $\S 1$ ). We let $\overline{\mathcal{J}} \rightarrow B$ denote a proper algebraic extension of $\mathcal{J}_{U} \rightarrow U$ (and more precisely of $\mathcal{J}_{U_{1}} \rightarrow U_{1}$ ) over $B$. Of course, such $\overline{\mathcal{J}}$ always exists; the goal of the paper is to establish the existence of a smooth $\overline{\mathcal{J}}$ which is flat over $B$, hence holomorphically symplectic.

Let $Y$ be a cubic 3-fold, and $l \subset Y$ a line (both $Y$ and $l$ need to satisfy some mild assumptions, to be specified in the text). The projection from $l$ realizes $Y$ as a conic 
bundle over $\mathbb{P}^{2}$ with a plane quintic $C$ as discriminant. The lines in $Y$ incident to $l$ are parameterized by a curve $\widetilde{C}$, which is a double cover (étale for general $l$ ) of $C$. We let $\mathcal{F} \rightarrow B$ denote the universal family of Fano surfaces, and $(\widetilde{\mathcal{C}}, \mathcal{C})$ the relative family of double covers over (a suitable open of) $\mathcal{F}$.

We will say that two hypersurface singularities $(V(f), 0) \subset \mathbb{C}^{n}$ and $(V(g), 0) \subset \mathbb{C}^{n+k}$ have the same type if they differ by a suspension, i.e. in suitable analytic coordinates $g\left(x_{1}, \ldots, x_{n+k}\right)=u \cdot\left(f\left(x_{1}, \ldots, x_{n}\right)+x_{n+1}^{2}+\ldots+x_{n+k}^{2}\right)$, with $u$ a unit in $\mathcal{O}_{\left(\mathbb{C}^{n+k}, 0\right)}$. The deformation spaces for singularities of the same type (and also the local monodromies if $k \equiv 0 \bmod 2)$ are naturally identified.

\section{Holomorphic 2-forms on Jacobian fibrations}

In [22], Donagi and Markman have performed the infinitesimal study of algebraically integrable systems, also called Lagrangian fibrations, which consist of a holomorphic family of complex tori, equipped with a non-degenerate $(2,0)$-form for which the fibers are Lagrangian. In this section, we provide an alternative way to construct a structure of Lagrangian or rather isotropic fibration on certain families of intermediate Jacobians. That is, we give a cycle and Hodge-theoretic argument to construct a closed holomorphic 2 -form vanishing on fibers of such families. The non-degeneracy of the holomorphic 2form needs to be checked by hand. However, a strong point of our construction is that it easily implies that the $(2,0)$-form extends to any algebraic smooth compactification of the family of intermediate Jacobians.

\subsection{The general case}

Let $X$ be a smooth projective variety of dimension $2 k$, and let $L$ be a line bundle on $X$. Assume that the smooth members $Y$ of the linear system $|L|$ have the following property:

$$
H^{p, q}(Y)=0, \quad p+q=2 k-1 \text { and }(p, q) \notin\{(k, k-1),(k-1, k)\} .
$$

Note that by [27] (see also [65, Vol. I, §12.1 and §12.2]) (1.1) is implied by the following property:

The Abel-Jacobi map $\Phi_{Y}: \mathrm{CH}^{k}(Y)_{\text {hom }} \rightarrow J^{2 k-1}(Y)$ is surjective.

Conversely, (1.1) should imply $(\star)$, according to the Hodge conjecture (see [66, $\S 2.2 .5]$ ). Under the assumption (1.1), the intermediate Jacobians $J(Y):=J^{2 k-1}(Y)$ are abelian varieties. Let $U \subset B:=|L|$ be the Zariski open set parameterizing smooth members, $\mathcal{Y} \subset B \times X$ be the universal family, and $\mathcal{Y}_{U}$ be its restriction to $U$. The family of 
intermediate Jacobians is under the same assumption a quasi-projective variety $\mathcal{J}_{U}$ with a smooth projective morphism

$$
\pi_{U}: \mathcal{J}_{U} \longrightarrow U
$$

with fiber $J\left(Y_{t}\right)$ over the point $t \in U$.

Let now $\eta \in H^{k+1, k-1}(X)$ and assume that $\left.\eta\right|_{Y}=0$ in $H^{k+1, k-1}(Y)$ for any smooth member $Y$ of $|L|$. (In our main application, where $X$ is the cubic 4 -fold and $k=2$, this assumption will be automatic, since $H^{k+1, k-1}\left(Y_{t}\right)$ will be 0 .) We are going to construct a holomorphic 2-form $\sigma_{U}$ on $\mathcal{J}_{U}$ associated with the above data and establish Theorem 1.2. We will first do this assuming $(\star)$ and will explain at the end how to adapt the proof when they only satisfy property (1.1).

Construction of the holomorphic 2-form. We denote by $u: \mathcal{Y}_{U} \rightarrow U$ the first projection, where $\mathcal{Y}_{U} \subset U \times X$ is the universal hypersurface. We have the folowing lemma.

Lemma 1.1. Assuming $(\star)$, there exists a codimension- $k$ cycle

$$
\mathcal{Z} \in \mathrm{CH}^{k}\left(\mathcal{J}_{U} \times_{U} \mathcal{Y}_{U}\right)_{\mathbb{Q}}
$$

such that the Betti cohomology class $\alpha:=[\mathcal{Z}] \in H^{2 k}\left(\mathcal{J}_{U} \times_{U} \mathcal{Y}_{U}, \mathbb{Q}\right)$, or rather its image $\alpha_{0}$ in $H^{0}\left(U, R^{2 k}\left(\pi_{u}, u\right)_{*} \mathbb{Q}\right)$, satisfies the condition that

$$
\alpha_{0}^{*}: R^{2 k-1} u_{*} \mathbb{Q} \longrightarrow R^{1} \pi_{U *} \mathbb{Q}
$$

is the natural isomorphism.

Proof. By the assumption $(\star)$, for each fiber $Y_{t}, t \in U$, there exist a smooth and projective variety $W_{t}$ and a family of codimension- $k$ cycles $\mathcal{T}_{t} \in \mathrm{CH}^{k}\left(W_{t} \times Y_{t}\right)$, such that $\left.\mathcal{T}_{t}\right|_{\{w\} \times Y_{t}}$ is homologically trivial, with the property that the Abel-Jacobi map

$$
\begin{aligned}
\Phi_{\mathcal{T}_{t}}: W_{t} & \longrightarrow J\left(Y_{t}\right), \\
w & \longmapsto \Phi_{Y_{t}}\left(\mathcal{T}_{t, w}\right),
\end{aligned}
$$

is surjective. It follows that there exists a codimension- $k$ cycle $\mathcal{Z}_{t}^{\prime} \in \mathrm{CH}^{k}\left(J\left(Y_{t}\right) \times Y_{t}\right)$ such that the Abel-Jacobi map

$$
\begin{aligned}
\Phi_{\mathcal{Z}_{t}^{\prime}}: J\left(Y_{t}\right) & \longrightarrow J\left(Y_{t}\right), \\
w & \longmapsto \Phi_{Y_{t}}\left(\mathcal{Z}_{t, w}^{\prime}\right),
\end{aligned}
$$


is $N$ times the identity of $J\left(Y_{t}\right)$ for some integer $N>0$. Indeed, we may assume that the surjective morphism $\Phi_{\mathcal{T}_{t}}: W_{t} \rightarrow J\left(Y_{t}\right)$ is generically finite of degree $N$, by replacing $W_{t}$ by a linear section if necessary. Then we set

$$
\mathcal{Z}_{t}^{\prime}:=\left(\Phi_{\mathcal{T}_{t}}, I d_{Y_{t}}\right)_{*} \mathcal{T}_{t} .
$$

The cycles $\mathcal{Z}_{t}^{\prime}$ have been defined fiberwise, but standard argument show that for an adequate choice of $N$ they can be constructed in family over a smooth generically finite cover $V$ of $U$ by spreading the original cycles $\mathcal{T}_{t, w}$. This provides a codimension- $k$ cycle $\mathcal{Z}^{\prime \prime} \in \mathrm{CH}^{k}\left(\mathcal{J}_{V} \times_{V} \mathcal{Y}_{V}\right)$ such that the class $\alpha^{\prime \prime}:=\left[\mathcal{Z}^{\prime \prime}\right]$ satisfies that

$$
\left(\alpha_{0}^{\prime \prime}\right)^{*}: R^{2 k-1} u_{*}^{\prime} \mathbb{Q} \longrightarrow R^{1} \pi_{*}^{\prime} \mathbb{Q}
$$

is $N$ times the natural isomorphism, where $u^{\prime}: \mathcal{Y}_{V} \rightarrow V$ and $\pi^{\prime}: \mathcal{J}_{V} \rightarrow V$ are the natural maps. We can choose a partial smooth completion $\bar{V}$ of $V$ such that the morphism $V \rightarrow U$ extends to a proper morphism $r: \bar{V} \rightarrow U$. We next extend the cycle $\mathcal{Z}^{\prime \prime}$ to a cycle $\overline{\mathcal{Z}^{\prime \prime}} \in \mathrm{CH}^{k}\left(\mathcal{J}_{\bar{V}} \times_{\bar{V}} \mathcal{Y}_{\bar{V}}\right)$. If $M=\operatorname{deg} r$, the cycle $\mathcal{Z}:=\tilde{r}_{*} \overline{\mathcal{Z}^{\prime \prime}} / M N \in \mathrm{CH}^{k}\left(\mathcal{J}_{U} \times_{U} \mathcal{Y}_{U}\right)$ satisfies the desired property, where $\tilde{r}: \mathcal{J}_{\bar{V}} \times_{\bar{V}} \mathcal{Y}_{\bar{V}} \rightarrow \mathcal{J}_{U} \times_{U} \mathcal{Y}_{U}$ is the natural degree- $M$ induced map.

Having the lemma, we now observe that there is a natural proper morphism

$$
q^{\prime}=(I d, q): \mathcal{J}_{U} \times_{U} \mathcal{Y}_{U} \longrightarrow \mathcal{J}_{U} \times X,
$$

where $q: \mathcal{Y}_{U} \rightarrow X$ is the second projection restricted to $\mathcal{Y}_{U} \subset U \times X$, and we thus get a codimension- $(k+1)$ cycle $\mathcal{Z}_{q}:=q_{*}^{\prime} \mathcal{Z} \in \mathrm{CH}^{k+1}\left(\mathcal{J}_{U} \times X\right)_{\mathbb{Q}}$ with Betti cohomology class $\left[\mathcal{Z}_{q}\right] \in$ $H^{2 k+2}\left(\mathcal{J}_{U} \times X, \mathbb{Q}\right)$ and Dolbeault cohomology class $\left[\mathcal{Z}_{q}\right]^{k+1, k+1} \in H^{k+1}\left(\mathcal{J}_{U} \times X, \Omega_{\mathcal{J}_{U} \times X}^{k+1}\right)$. For any $\eta \in H^{k-1}\left(X, \Omega_{X}^{k+1}\right)$, the corresponding class $\sigma_{U} \in H^{0}\left(\mathcal{J}_{U}, \Omega_{J_{U}}^{2}\right)$ is defined by the formula

$$
\sigma_{U}=\left(\left[\mathcal{Z}_{q}\right]^{k+1, k+1}\right)^{*}(\eta),
$$

where $\left(\left[\mathcal{Z}_{q}\right]^{k+1, k+1}\right)^{*}: H^{k-1}\left(X, \Omega_{X}^{k+1}\right) \rightarrow H^{0}\left(\mathcal{J}_{U}, \Omega_{J_{U}}^{2}\right)$ is defined by

$$
\left(\left[\mathcal{Z}_{q}\right]^{k+1, k+1}\right)^{*}(\omega)=\operatorname{pr}_{1 *}\left(\left[\mathcal{Z}_{q}\right]^{k+1, k+1} \cup \operatorname{pr}_{2}^{*} \omega\right),
$$

the $\mathrm{pr}_{i}$ 's being the two projections defined on $\mathcal{J}_{U} \times X$. This completes the construction of the form $\sigma_{U}$.

The following notation will be used below: as we have $\left.\eta\right|_{Y_{t}}=0$ in $H^{k-1}\left(\Omega_{Y_{t}}^{k+1}\right)$ and $H^{k-2}\left(Y_{t}, \Omega_{Y_{t}}^{k+1}\right)=0$ by (1.1), $\eta$ determines a class

$$
\tilde{\eta}_{t} \in H^{k-1}\left(Y_{t}, \Omega_{Y_{t}}^{k}(-L)\right)
$$

using the exact sequence

$$
\left.0 \longrightarrow \Omega_{Y_{t}}^{k}(-L) \longrightarrow \Omega_{X}^{k+1}\right|_{Y_{t}} \longrightarrow \Omega_{Y_{t}}^{k+1} \longrightarrow 0 .
$$


THEOREM 1.2. The holomorphic 2-form

$$
\sigma_{U} \in H^{0}\left(\mathcal{J}_{U}, \Omega_{\mathcal{J}_{U}}^{2}\right)
$$

constructed above satisfies the following properties:

(i) The fibers of the fibration $\pi_{U}: \mathcal{J}_{U} \rightarrow U$ are isotropic for $\sigma_{U}$

(ii) At any point $t \in U$, the map $\lrcorner \sigma_{t}: T_{U, t} \rightarrow H^{0}\left(\mathcal{J}_{t}, \Omega_{\mathcal{J}_{t}}\right)=H^{k-1}\left(Y_{t}, \Omega_{Y_{t}}^{k}\right)$ induced by $\sigma_{U}$ using (i) identifies with the multiplication map

$$
T_{U, t}=H^{0}\left(Y_{t},\left.L\right|_{Y_{t}}\right) \longrightarrow H^{k-1}\left(Y_{t}, \Omega_{Y_{t}}^{k}\right)
$$

by the class $\tilde{\eta}_{t}$ of $(1.3)$.

(iii) For any smooth algebraic variety $\overline{\mathcal{J}}$ containing $\mathcal{J}_{U}$ as a Zariski open set, the 2 -form $\sigma_{U}$ extends to a holomorphic 2-form on $\overline{\mathcal{J}}$.

(iv) The 2-form $\sigma_{U}$ is closed.

Proof. Denoting by $p_{1}: \mathcal{J}_{U} \times{ }_{U} \mathcal{Y}_{U} \rightarrow \mathcal{J}_{U}$ the first projection and by $p_{2}: \mathcal{J}_{U} \times_{U} \mathcal{Y}_{U} \rightarrow \mathcal{Y}_{U}$ the second one, formula (1.2) gives as well, using the projection formula and the fact that $[\mathcal{Z}]_{q}^{k+1, k+1}=q_{*}^{\prime}\left([\mathcal{Z}]^{k, k}\right)$,

$$
\sigma_{U}=\left([\mathcal{Z}]^{k, k}\right)^{*}\left(q^{*} \eta\right),
$$

where $\left.\left([\mathcal{Z}]^{k, k}\right)^{*}: H^{k-1}\left(\mathcal{Y}_{U}, \Omega_{\mathcal{Y}_{U}}^{k+1}\right)\right) \rightarrow H^{0}\left(\mathcal{J}_{U}, \Omega_{J_{U}}^{2}\right)$ is defined by

$$
\left([\mathcal{Z}]^{k, k}\right)^{*}(\omega)=p_{1 *}\left([\mathcal{Z}]^{k, k} \cup p_{2}^{*} \omega\right) .
$$

(i) This follows immediately from (1.4) which gives, for $t \in U$,

$$
\left.\sigma_{U}\right|_{\mathcal{J}_{t}}=\left(\left.[\mathcal{Z}]^{k, k}\right|_{\mathcal{J}_{t} \times Y_{t}}\right)^{*}\left(\left.\eta\right|_{Y_{t}}\right)
$$

and from the fact that $\left.\eta\right|_{Y_{t}}=0$ in $H^{k+1, k-1}\left(Y_{t}\right)$ by assumption. (Here we identify the fiber $Y_{t}$ of the universal family and its image in $X$.)

(iii) We observe that if $\overline{\mathcal{J}} \supsetneq \mathcal{J}_{U}$ is a smooth algebraic partial compactification of $\mathcal{J}_{U}$, the cycle $\mathcal{Z}_{q}$ extends to a cycle $\overline{\mathcal{Z}}_{q} \in \mathrm{CH}^{k+1}(\overline{\mathcal{J}} \times X)$, so that its cohomology class $\left[\mathcal{Z}_{q}\right]^{k+1, k+1}$ extends to a class

$$
\left[\overline{\mathcal{Z}}_{q}\right]^{k+1, k+1} \in H^{k+1}\left(\overline{\mathcal{J}} \times X, \Omega_{\overline{\mathcal{J}} \times X}^{k+1}\right) .
$$

It thus follows that the form $\sigma_{U}$ extends to a 2 -form $\sigma \in H^{0}\left(\overline{\mathcal{J}}, \Omega \frac{2}{J}\right)$ given by the formula

$$
\sigma=\left(\left[\overline{\mathcal{Z}}_{q}\right]^{k+1, k+1}\right)^{*}(\eta)
$$


(iv) This is an immediate consequence of (iii). Indeed, choosing a smooth projective compactification $\overline{\mathcal{J}} \supseteq \mathcal{J}_{U}$ of $\mathcal{J}_{U}$, the 2-form $\sigma_{U}$ extends by (iii) to a holomorphic 2-form $\sigma$ on $\overline{\mathcal{J}}$. The 2-form $\sigma$ is closed, hence the original form $\sigma_{U}$ is closed.

(ii) Consider the class $q^{*} \eta \in H^{k-1}\left(\mathcal{Y}_{U}, \Omega_{\mathcal{Y}_{U}}^{k+1}\right)$. As it vanishes on fibers $Y_{t}$ of $u, t \in U$, it provides for any $t \in U$ a morphism

$$
\operatorname{int}(\cdot) q^{*} \eta: T_{U, t} \longrightarrow H^{k-1}\left(Y_{t}, \Omega_{Y_{t}}^{k}\right)
$$

which for $k=1$ is simply obtained by taking interior product of the 2 -form $\eta$ with a local lift of the considered tangent vector on the base, and for arbitrary $k$ is constructed as follows: the cotangent bundle sequence of $u$,

$$
0 \longrightarrow \Omega_{U, t} \otimes \mathcal{O}_{Y_{t}} \longrightarrow \Omega_{\left.\mathcal{Y}_{U}\right|_{Y_{t}}} \longrightarrow \Omega_{Y_{t}} \longrightarrow 0,
$$

induces an exact sequence

$$
\left.0 \longrightarrow \Omega_{U, t} \otimes \Omega_{Y_{t}}^{k} \longrightarrow \Omega_{\mathcal{Y}_{U}}^{k+1}\right|_{Y_{t}} /\left.L^{2} \Omega_{\mathcal{Y}_{U}}^{k+1}\right|_{Y_{t}} \longrightarrow \Omega_{Y_{t}}^{k+1} \longrightarrow 0
$$

where $L^{2} \Omega_{\left.\mathcal{Y}_{U}\right|_{Y_{t}}}^{k+1} \subset \Omega_{\left.\mathcal{Y}_{U}\right|_{Y_{t}}}^{k+1}$ is the sub-bundle $u^{*} \Omega_{U, t}^{2} \wedge \Omega_{\left.\mathcal{Y}_{U}\right|_{Y_{t}}}^{k-1}$. From (1.7), using the fact that $H^{k-2}\left(Y_{t}, \Omega_{Y_{t}}^{k+1}\right)=0$, we deduce that the class $\left.q^{*} \eta\right|_{Y_{t}} \in H^{k-1}\left(Y_{t},\left.\Omega_{\mathcal{Y}_{U}}^{k+1}\right|_{Y_{t}}\right)$ lifts to a unique class $\widetilde{q^{*} \eta_{t}}$ in $\Omega_{U, t} \otimes H^{k-1}\left(Y_{t}, \Omega_{Y_{t}}^{k}\right)=\operatorname{Hom}\left(T_{U, t}, H^{k-1}\left(Y_{t}, \Omega_{Y_{t}}^{k}\right)\right)$, giving the desired morphism $\operatorname{int}(\cdot) q^{*} \eta$ of (1.6). We now use formula (1.4), which makes obvious that, for any $t \in U$ and $v \in T_{U, t}$,

$$
v\lrcorner \sigma_{U}=\left(\left.[\mathcal{Z}]^{k, k}\right|_{\mathcal{J}_{t} \times Y_{t}}\right)^{*}\left(\operatorname{int}(v) q^{*} \eta\right) \quad \text { in } H^{0}\left(\mathcal{J}_{t}, \Omega_{\mathcal{J}_{t}}\right) .
$$

In the right-hand side, we recall that, by construction, the morphism

$$
\left(\left.[\mathcal{Z}]^{k, k}\right|_{\mathcal{J}_{t} \times Y_{t}}\right)^{*}: H^{k}\left(Y_{t}, \Omega_{Y_{t}}^{k-1}\right) \longrightarrow H^{0}\left(\mathcal{J}_{t}, \Omega_{\mathcal{J}_{t}}\right)
$$

is the natural isomorphism. It thus only remains to analyze the morphism $\operatorname{int}(\cdot) q^{*} \eta$. We observe now that the cotangent bundle sequence (1.7) is compatible with the conormal bundle sequence of $Y_{t}$ in $X$, since via the differential $q^{*}$ of the morphism $q: \mathcal{Y} \rightarrow X$, we get the following commutative diagram:

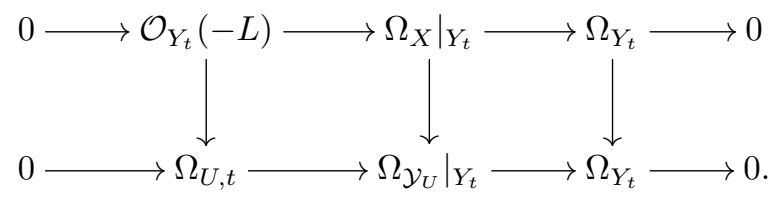


The first vertical map is the natural inclusion dual to the evaluation map $T_{U, t}=$ $\left.H^{0}\left(Y_{t},\left.L\right|_{Y_{t}}\right) \rightarrow L\right|_{Y_{t}}$. Taking $(k+1)$-th exterior powers, we get the following commutative diagram:

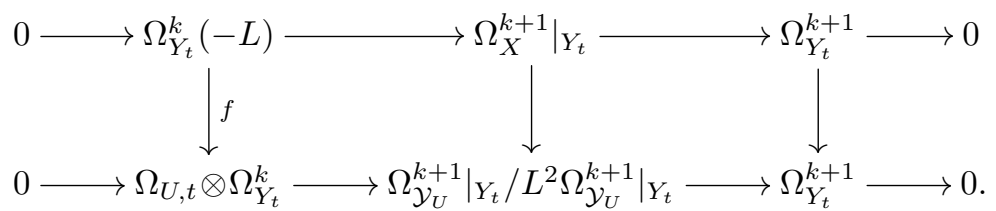

It clearly follows from the commutativity of diagram (1.10) that

$$
f\left(\tilde{\eta}_{t}\right)=\widetilde{q^{*} \eta_{t}} \quad \text { in } \Omega_{U, t} \otimes H^{k-1}\left(Y_{t}, \Omega_{Y_{t}}^{k}\right)
$$

(where $\tilde{\eta}_{t}$ is as in (1.3)). The proof of (ii) is now a consequence of (1.8) and (1.11). Indeed, for any $v \in T_{U, t}$ we have

$$
v\lrcorner \sigma_{U}=\left(\left.[\mathcal{Z}]^{k, k}\right|_{\mathcal{J}_{t} \times Y_{t}}\right)^{*}\left(\operatorname{int}(v) q^{*} \eta\right)=\left(\left.[\mathcal{Z}]^{k, k}\right|_{\mathcal{J}_{t} \times Y_{t}}\right)^{*}\left(\widetilde{q^{*}} \eta_{t}(v)\right) \quad \text { in } H^{0}\left(\mathcal{J}_{t}, \Omega_{\mathcal{J}_{t}}\right),
$$

where the first equality is (1.8) and the second equality is by definition of $\widetilde{q^{*} \eta_{t}}$. The equality (1.11) then tells that

$$
\widetilde{q^{*}} \eta_{t}(v)=v \tilde{\eta}_{t},
$$

where, on the right, $v$ is seen as an element of $H^{0}\left(\mathcal{O}_{Y_{t}}(-L)\right)$ and $v \tilde{\eta}_{t}$ is the product of $v$ and $\eta_{t}$. The proof is thus finished, since $\left(\left.[\mathcal{Z}]^{k, k}\right|_{\mathcal{J}_{t} \times Y_{t}}\right)^{*}$ is the natural identification between $H^{k-1}\left(Y_{t}, \Omega_{Y_{t}}^{k}\right)$ and $H^{0}\left(\mathcal{J}_{t}, \Omega_{\mathcal{J}_{t}}\right)$.

The construction of the 2-form $\sigma_{U}$ and the proof of Theorem 1.2, assuming property $(\star)$, are now complete. We conclude this section explaining how to modify the arguments to get the same results only assuming (1.1). If we examine the proofs given above, we see that the key tool is the algebraic cycle $\mathcal{Z} \in \mathrm{CH}^{k}\left(\mathcal{J}_{U} \times_{U} \mathcal{Y}_{U}\right)_{\mathbb{Q}}$ and its image $\mathcal{Z}_{q} \in \mathrm{CH}^{k+1}\left(\mathcal{J}_{U} \times X\right)_{\mathbb{Q}}$. These cycles appear only through their Dolbeault classes $[\mathcal{Z}]^{k, k}$, $\left[\mathcal{Z}_{q}\right]^{k+1, k+1}$, which are better seen, after extensions to smooth projective varieties, as Hodge classes. In the absence of the cycle $\mathcal{Z}$ that we constructed using the assumption $(\star)$ we still have the desired Hodge classes, as follows from the following lemma. Below, a Hodge class on a smooth quasi-projective variety $Y$ is by definition the restriction of a Hodge class on a smooth projective compactification $\bar{Y}$ of $Y$. The set of Hodge classes on $Y$ does not depend on the compactification $\bar{Y}$. In fact, according to Deligne [21], Hodge classes in $H^{2 k}(Y, \mathbb{Q})$ are identified with $\operatorname{Hdg}^{2 k}\left(W_{2 k} H^{2 k}(Y, \mathbb{Q})\right)$, where $W_{2 k} H^{2 k}(Y, \mathbb{Q})$ is the smallest weight part of $H^{2 k}(Y, \mathbb{Q})$, which is also the image of the restriction map $H^{2 k}(\bar{Y}, \mathbb{Q}) \rightarrow H^{2 k}(Y, \mathbb{Q})$ for any smooth projective compactification $\bar{Y}$ of $Y$. Hodge classes $\alpha \in \operatorname{Hdg}^{2 k}\left(H^{2 k}(Y, \mathbb{Q})\right)$ have a Dolbeault counterpart $\alpha^{k, k} \in H^{k}\left(Y, \Omega_{Y}^{k}\right)$ (which usually does not determine $\alpha$ in the non-projective situation). 
Lemma 1.3. Let $X, k$ and $L$ be as above, satisfying condition (1.1). Then, there exists a Hodge class $\alpha \in \operatorname{Hdg}^{2 k}\left(\mathcal{J}_{U} \times_{U} \mathcal{Y}_{U}, \mathbb{Q}\right)$ with the property that the class

$$
\alpha_{0} \in H^{0}\left(U, R^{2 k}\left(\pi_{U}, u\right)_{*} \mathbb{Q}\right)
$$

induces the natural isomorphism $H^{2 k-1}\left(Y_{t}, \mathbb{Q}\right) \cong H^{1}\left(\mathcal{J}_{t}, \mathbb{Q}\right)$ at any point $t \in U$.

Proof. The only observation to make is that the canonical isomorphism

$$
H^{2 k-1}\left(Y_{t}, \mathbb{Q}\right) \cong H^{1}\left(\mathcal{J}_{t}, \mathbb{Q}\right)
$$

is an isomorphism of Hodge structures of bidegree $(-k+1,-k+1)$, by the vanishing condition (1.1). Such an isomorphism of Hodge structures provides a degree $2 k$ Hodge class $\alpha_{t}$ on the product $\mathcal{J}_{t} \times Y_{t}$. We thus have a section of the local system $R^{2 k}\left(\pi_{U}, u\right)_{*} \mathbb{Q}$ which is a Hodge class at any point $t$ of $U$. Deligne's global invariant cycle theorem [21] then says that for any smooth projective compactification $M$ of $\mathcal{J}_{U} \times_{U} \mathcal{Y}_{U}$, there exists a cohomogy class $\beta \in H^{2 k}(M, \mathbb{Q})$ such that $\left.\beta\right|_{\mathcal{J}_{t} \times Y_{t}}=\alpha_{t}$ for any $t \in U$. Using the facts that $\alpha_{t}$ is a Hodge class and the restriction morphism $H^{2 k}(M, \mathbb{Q}) \rightarrow H^{2 k}\left(\mathcal{J}_{t} \times Y_{t}, \mathbb{Q}\right)$ is a morphism of polarized Hodge structures, the semi-simplicity of the category of polarized rational Hodge structures allows us to conclude that the class $\beta$ can be chosen to be Hodge on $M$ (see $[66, \S 2.2 .1])$. The restriction of $\beta$ to $\mathcal{J}_{U} \times_{U} \mathcal{Y}_{U}$ is then the desired Hodge class $\alpha$ on $\mathcal{J}_{U} \times_{U} \mathcal{Y}_{U}$.

This concludes the proof of Theorem 1.2 assuming only (1.1). We conclude this section observing that, except for (ii), we did not use the condition that $\mathcal{Y}$ is the universal family of smooth divisors in $X$. Any smooth projective family mapping to $X$, or even only having a correspondence $Z$ with $X$, with fibers satisfying condition (1.1) will do. In practice, (ii) gives a way of deciding whether the constructed 2-form is degenerate or not. So our arguments prove more generally the following variant of Theorem 1.2:

THEOREM 1.4. Let $X$ be a smooth projective variety of dimension $n$ and $f: \mathcal{Y} \rightarrow U$ be a smooth projective morphism between smooth quasi-projective varieties. Let $l$ and $k$ be integers and let $Z \in \mathrm{CH}^{n-l+k-1}(\mathcal{Y} \times X)_{\mathbb{Q}}$ be a codimension- $(n-l+k-1)$ cycle. Assume that the fibers $Y_{t}$ of $f$ satisfy condition (1.1) for the given integer $k$. Let $\pi_{U}: \mathcal{J}_{U} \rightarrow U$ be the family of intermediate Jacobians $J^{2 k-1}\left(Y_{t}\right), t \in U$. Then

(i) For any class $\eta \in H^{l+2, l}(X)$ such that $\left.Z^{*} \eta\right|_{Y_{t}}=0$ in $H^{k+1, k-1}\left(Y_{t}\right)$ for any $t$ in $U$, there is a closed $(2,0)$-form $\sigma_{U} \in H^{0}\left(\mathcal{J}_{U}, \Omega_{\mathcal{J}_{U}}^{2}\right)$ for which the fibers of $\pi_{U}$ are isotropic.

(ii) For any smooth algebraic partial compactification $\mathcal{J}_{U} \subset \overline{\mathcal{J}}$, the $(2,0)$-form $\sigma_{U}$ extends to a $(2,0)$-form $\sigma$ on $\overline{\mathcal{J}}$. 
Example 1.5. In [36], Iliev and Manivel construct a Lagrangian fibration structure on the family of intermediate Jacobians of smooth cubic 5-folds containing a given cubic 4 -fold $X$. We recover the closed $(2,0)$-form as an application of Theorem 1.4: The family $\mathcal{Y}_{U}$ in this case is the universal family of these cubic 5 -folds and the integer $k$ is 3 . The cycle $Z \subset X \times \mathcal{Y}_{U} \cong \mathcal{Y}_{U} \times X$ is isomorphic to $X \times U$ and will be given by the embedding of $X$ in $Y_{t}$ for any $t \in U$, hence we have $l=1, n=4, n-l+k-1=5$ in this case. Of course, some more work as in Theorem 1.2 (ii) is needed to show that the $(2,0)$-form is nondegenerate, but our approach shows that this forms extends to any smooth projective compactification.

\subsection{The case of the cubic 4 -fold}

The paper will be devoted to the case where $X \subset \mathbb{P}^{5}$ is a cubic 4-fold, $L=\mathcal{O}_{X}(1)$ and $k=2$. One has $\operatorname{dim} H^{3,1}(X)=1$ by Griffiths' theory, and a generator $\eta$ of $H^{3,1}(X)$ provides thus by Theorem 1.2 a $(2,0)$-form $\sigma_{U}$ on the family of intermediate Jacobians of smooth hyperplane sections of $X$. We have the following result.

Proposition 1.6. If $X$ is a smooth cubic 4-fold, the holomorphic 2-form $\sigma_{U}$ is non-degenerate on $\mathcal{J}_{U}$.

Proposition 1.6 already appears in [22], [36], [46], [43]. The proof given here is slightly different, being an easy application of Theorem 1.2.

Proof of Proposition 1.6. We apply Theorem 1.2. In the case of the family of hyperplane sections of a cubic 4-fold, the base $U$ and the fiber $J\left(Y_{t}\right)$ of the family $\mathcal{J}_{U} \rightarrow U$ are of dimension 5. The 2 -form $\sigma_{U}$ vanishes along the fibers of $\pi_{U}$, and in order to prove that it is non-degenerate, it suffices to show that at any point $t \in U$, the map \lrcorner$(\cdot) \sigma_{U}: T_{U, t} \rightarrow H^{0}\left(\mathcal{J}_{t}, \Omega_{\mathcal{J}_{t}}\right)$ is an isomorphism. Theorem 1.2 (ii) tells us that $\lrcorner(\cdot) \sigma_{U}$ is the following map: the generator $\eta$ induces for each point $t \in U$ a class $\tilde{\eta}_{t} \in H^{1}\left(Y_{t}, \Omega_{Y_{t}}^{2}(-1)\right)$. Then, using the identification $H^{0}\left(\mathcal{J}_{t}, \Omega_{\mathcal{J}_{t}} \cong H^{1}\left(Y_{t}, \Omega_{Y_{t}}^{2}\right)\right.$, we have that

$$
\lrcorner(\cdot) \sigma_{U}: T_{U, t}=H^{0}\left(Y_{t}, \mathcal{O}_{Y_{t}}(1)\right) \longrightarrow H^{1}\left(Y_{t}, \Omega_{Y_{t}}^{2}\right)
$$

is the multiplication map by $\tilde{\eta}_{t}$. So the statement of Proposition 1.6 is the following.

Claim. For any $t \in U$, the class $\tilde{\eta}_{t} \in H^{1}\left(Y_{t}, \Omega_{Y_{t}}^{2}(-1)\right)$ induces an isomorphism

$$
H^{0}\left(Y_{t}, \mathcal{O}_{Y_{t}}(1)\right) \longrightarrow H^{1}\left(Y_{t}, \Omega_{Y_{t}}^{2}\right) \text {. }
$$

The proof of the claim is a consequence of the following lemma. 
LEMma 1.7. (i) The class $\tilde{\eta}_{t} \in H^{1}\left(Y_{t}, \Omega_{Y_{t}}^{2}(-1)\right)$ is a non-zero multiple of the extension class e of the normal bundle sequence

$$
\left.0 \longrightarrow T_{Y_{t}} \longrightarrow T_{\mathbb{P}^{4}}\right|_{Y_{t}} \longrightarrow \mathcal{O}_{Y_{t}}(3) \longrightarrow 0
$$

using the natural identification $\Omega_{Y_{t}}^{2}(-1) \cong T_{Y_{t}}(-3)$.

(ii) The extension class e has the property that the multiplication map by

$$
e: H^{0}\left(Y_{t}, \mathcal{O}_{Y_{t}}(1)\right) \longrightarrow H^{1}\left(Y_{t}, \Omega_{Y_{t}}^{2}\right)
$$

is an isomorphism.

Proof. (ii) is Griffiths' residue isomorphism (see [65, Vol. II, §6.1.3]) and in this case, the statement immediately follows from the exact sequence (1.13) and the fact that $H^{0}\left(Y_{t},\left.T_{\mathbb{P}^{4}}\right|_{Y_{t}}(-2)\right)=0$, and $H^{2}\left(Y_{t}, T_{Y_{t}}(-2)\right)=0$. As for (i), this simply follows from the fact that the class $\tilde{\eta}_{t} \in H^{1}\left(Y_{t}, \Omega_{Y_{t}}^{2}(-1)\right)$ is non-zero because $\eta \neq 0$, and $H^{1}\left(X, \Omega_{X}^{3}(-1)\right)=0$, so that $\left.\eta\right|_{Y_{t}} \neq 0$. On the other hand, $H^{1}\left(Y_{t}, \Omega_{Y_{t}}^{2}(-1)\right)=H^{1}\left(Y_{t}, T_{Y_{t}}(-3)\right)$ is 1-dimensional, as follows from the normal bundle sequence (1.13).

The proof of Proposition 1.6 is finished.

\subsection{Another example: quadric sections of cubic 4-folds}

Note that the cubic 4-fold $X$ has another family of smooth divisors $Y \subset X$ satisfying condition (1.1), namely the smooth complete intersections $Q \cap X$, where $Q$ is a quadric in $\mathbb{P}^{5}$. The corresponding family $\mathcal{J}_{Q} \rightarrow U_{Q}$ of intermediate Jacobians has a basis $U_{Q}$ of dimension $20=h^{0}\left(\mathbb{P}^{5}, \mathcal{O}_{\mathbb{P}^{5}}(2)\right)-1$ and fibers of dimension 20. Theorem 1.2 shows that $\mathcal{J}_{Q}$ has a closed holomorphic 2-form $\sigma_{Q}$ which extends to any smooth algebraic compactification $\overline{\mathcal{J}}_{Q}$ of $\mathcal{J}_{Q}$. However the 2 -form in this case is only generically non-degenerate.

LEMMA 1.8. The 2-form $\sigma_{Q}$ is non-degenerate along a fiber $\mathcal{J}_{Q, t}=J\left(Y_{t}\right)$, where $Y_{t}=Q_{t} \cap X$, if and only if the quadric $Q_{t}$ is non-degenerate.

Proof. The generator $\eta$ of $H^{1}\left(X, \Omega_{X}^{3}\right)=H^{1}\left(X, T_{X}(-3)\right)$ is the extension class of the normal bundle sequence

$$
\left.0 \longrightarrow T_{X} \longrightarrow T_{\mathbb{P}^{5}}\right|_{X} \longrightarrow \mathcal{O}_{X}(3) \longrightarrow 0 .
$$

Restricting to $Y_{t}=Q_{t} \cap X$, we get the exact sequence

$$
\left.\left.0 \longrightarrow T_{X}\right|_{Y_{t}} \longrightarrow T_{\mathbb{P}^{5}}\right|_{Y_{t}} \longrightarrow \mathcal{O}_{Y_{t}}(3) \longrightarrow 0,
$$


whose extension class must come from the extension class $e_{t}$ of the normal bundle sequence

$$
\left.0 \longrightarrow T_{Y_{t}} \longrightarrow T_{Q}\right|_{Y_{t}} \longrightarrow \mathcal{O}_{Y_{t}}(3) \longrightarrow 0
$$

of $Y_{t}$ in $Q_{t}$. In other words, the class $\tilde{\eta}_{t} \in H^{1}\left(\Omega_{Y_{t}}^{2}(-2)\right)=H^{1}\left(Y_{t}, T_{Y_{t}}(-3)\right)$ is nothing but the extension class $e_{t}$. It follows that the multiplication map

$$
\tilde{\eta}_{t}: H^{0}\left(Y_{t}, \mathcal{O}_{Y_{t}}(2)\right) \longrightarrow H^{1}\left(Y_{t}, \Omega_{Y_{t}}^{2}\right)
$$

identifies with the map $H^{0}\left(Y_{t}, \mathcal{O}_{Y_{t}}(2)\right) \rightarrow H^{1}\left(Y_{t}, \Omega_{Y_{t}}^{2} \cong H^{1}\left(Y_{t}, T_{Y_{t}}(-1)\right)\right.$ induced by the exact sequence (1.14) twisted by $\mathcal{O}_{Y_{t}}(-1)$. Looking at the long exact sequence associated with (1.14), we find that this map is an isomorphism if and only if $H^{0}\left(Y_{t},\left.T_{Q_{t}}\right|_{Y_{t}}(-1)\right)=0$. But $H^{0}\left(Y_{t},\left.T_{Q_{t}}\right|_{Y_{t}}(-1)\right)=0$ if and only if $Q_{t}$ is not singular.

\subsection{Extensions to nodal fibers}

Let $X$ be a smooth cubic 4-fold, and $\eta \in H^{1}\left(X, \Omega_{X}^{3}\right)$ be a generator of $H^{1}\left(X, \Omega_{X}^{3}\right)$. We use as before the notation $\mathcal{Y} \rightarrow B$ and $\mathcal{Y}_{U} \rightarrow U$ for the universal family of hyperplane sections of $X$. Let $U_{1} \subset\left|\mathcal{O}_{X}(1)\right|$ be the Zariski open set parameterizing hyperplane sections of $X$ with at most one ordinary double point. The Jacobian fibration $\pi_{U}: \mathcal{J}_{U} \rightarrow U$ has a flat projective extension $\pi_{U_{1}}: \mathcal{J}_{U_{1}} \rightarrow U_{1}$ with smooth total space (see Lemma 5.2 for the smoothness statement). As the vanishing cycle of the degeneration is not trivial at a point $t \in U_{1} \backslash U$, the fiber of $\pi_{U_{1}}$ over $t$ is a singular compactification of a $\mathbb{C}^{*}$-bundle over $J\left(\widetilde{Y}_{t}\right)$, where $\widetilde{Y}_{t}$ is the desingularization of $Y_{t}$ obtained by blowing-up the node. We will denote below by $\mathcal{J}_{U_{1}}^{\circ}$ the quasi-abelian part of $\mathcal{J}_{U_{1}}$. Note that $\mathcal{J}_{U_{1}}^{\circ} \subset \mathcal{J}_{U_{1}}$ has a complement of codimension 2, consisting of the singular loci of the compactified Jacobians over $U_{1} \backslash U$. By Theorem 1.2 (iii), the 2-form $\sigma_{U}$ extends to a 2 -form $\sigma_{U_{1}}$ on $\mathcal{J}_{U_{1}}$, for which the fibers of $\pi_{U_{1}}$ are isotropic by Theorem $1.2(\mathrm{i})$ (and the fact that the fibers of $\pi_{U_{1}}$ are equidimensional). Next, the smooth locus $J\left(Y_{t}\right)_{\mathrm{reg}}=\mathcal{J}_{U_{1}, t}^{\circ}$ is a quasi-abelian variety with cotangent space isomorphic to $H^{1}\left(\widetilde{Y}_{t}, \Omega_{\widetilde{Y}_{t}}^{2}\left(\log E_{Y}\right)\right)$, where $E_{Y}$ is the exceptional divisor of the resolution $\widetilde{Y}_{t} \rightarrow Y_{t}$.

Our goal in this section is to reprove the following result which can be found in $[22$, $\S 8.5 .2]$.

Proposition 1.9. The extended 2-form $\sigma_{U_{1}}$ is everywhere non-degenerate on $\mathcal{J}_{U_{1}}^{\circ}$, hence also on $\mathcal{J}_{U_{1}}$.

As an immediate corollary, we get the following result.

Corollary 1.10. Assume that $\mathcal{J}_{U_{1}}$ has a smooth compactification $\overline{\mathcal{J}}$ with boundary $\overline{\mathcal{J}} \backslash \mathcal{J}_{U_{1}}$ of codimension $\geqslant 2$ in $\overline{\mathcal{J}}$. Then $\overline{\mathcal{J}}$ is holomorphically symplectic. 
Proof. Indeed, the extended 2-form, being non-degenerate away from a codimension2 closed analytic subset, is everywhere non-degenerate.

The proof of Proposition 1.9 is based on Lemma 1.11 below. For $t \in U_{1} \backslash U$, we have the inclusions

$$
\widetilde{Y_{t}} \subset \widetilde{X} \subset \widetilde{\mathbb{P}^{5}}
$$

where $\widetilde{X}$ (resp. $\widetilde{\mathbb{P}^{5}}$ ) is the blow-up of $X$ (resp. $\mathbb{P}^{5}$ ) at the singular point of $Y_{t}$. We denote

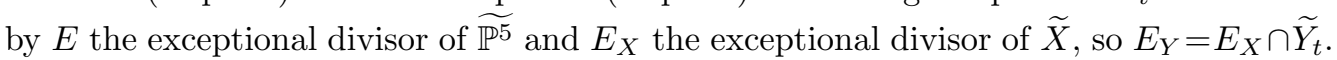
As $\widetilde{X}$ is transverse to the exceptional divisor $E$ of $\widetilde{\mathbb{P}^{5}}$ and belongs to the linear system $\left|\mathcal{O}_{\widetilde{\mathbb{P}^{5}}}(3)(-E)\right|$, we have a logarithmic tangent bundles sequence

$$
\left.0 \longrightarrow T_{\widetilde{X}}\left(\log E_{X}\right) \longrightarrow T_{\widetilde{\mathbb{P}} 5}(\log E)\right|_{\widetilde{X}} \longrightarrow \mathcal{O}_{\widetilde{X}}(3)\left(-E_{X}\right) \longrightarrow 0 .
$$

Here we recall that the logarithmic tangent bundle of a variety equipped with a smooth divisor $D$ is the dual of the logarithmic cotangent bundle determined by $D$ and can be defined as the sheaf of vector fields tangent to $D$ along $D$. As $E_{X} \cap \widetilde{Y}_{t}=E_{Y}$, we also get natural inclusions for any $l$ :

$$
\left.T_{\widetilde{Y_{t}}}\left(\log E_{Y}\right)\left(l E_{Y}\right)(-3) \subset T_{\widetilde{X}}\left(\log E_{X}\right)\left(l E_{X}\right)(-3)\right|_{\widetilde{Y}_{t}} .
$$

LEMma 1.11. The induced map $\lrcorner \sigma_{U_{1}, t}: T_{U_{1}, t} \rightarrow H^{1}\left(\widetilde{Y}_{t}, \Omega_{\widetilde{Y}_{t}}^{2}\left(\log E_{Y}\right)\right)$ is constructed as follows:

(i) The extension class $e \in H^{1}\left(T_{\widetilde{X}}\left(\log E_{X}\right)\left(E_{X}\right)(-3)\right)$ of $(1.15)$ maps naturally to an element $e^{\prime} \in H^{1}\left(T_{\widetilde{X}}\left(\log E_{X}\right)\left(2 E_{X}\right)(-3)\right)$, which restricted to $\widetilde{Y}_{t}$ comes via (1.16) from a uniquely defined element

$$
e_{Y} \in H^{1}\left(\widetilde{Y}_{t}, T_{\widetilde{Y}_{t}}\left(\log E_{Y}\right)\left(2 E_{Y}\right)(-3)\right) .
$$

(ii) One has $T_{\widetilde{Y}_{t}}\left(\log E_{Y}\right)\left(2 E_{Y}\right)(-3)=\Omega_{\widetilde{Y}_{t}}^{2}\left(\log E_{Y}\right)(-1)$, thus

$$
e_{Y} \in H^{1}\left(\widetilde{Y}_{t}, \Omega_{\widetilde{Y}_{t}}^{2}\left(\log E_{Y}\right)(-1)\right) \text {. }
$$

(iii) The interior product $\lrcorner \sigma_{U_{1}, t}: T_{U_{1}, t} \rightarrow H^{1}\left(\widetilde{Y}_{t}, \Omega_{\widetilde{Y}_{t}}^{2}\left(\log E_{Y}\right)\right)$ is given by multiplication $H^{0}\left(\widetilde{Y}_{t}, \mathcal{O}_{\widetilde{Y_{t}}}(1)\right) \stackrel{e_{Y}}{\longrightarrow} H^{1}\left(\widetilde{Y}_{t}, \Omega_{\widetilde{Y}_{t}}^{2}\left(\log E_{Y}\right)\right)$.

Proof. (i) We write the logarithmic normal bundle sequence for $\widetilde{Y}_{t} \subset \tilde{X}$ :

$$
\left.0 \longrightarrow T_{\widetilde{Y_{t}}}\left(\log E_{Y}\right) \longrightarrow T_{\widetilde{X}}\left(\log E_{X}\right)\right|_{\widetilde{Y}_{t}} \longrightarrow \mathcal{O}_{\widetilde{Y}_{t}}(1)\left(-2 E_{Y}\right) \longrightarrow 0
$$

which we twist by $\mathcal{O}_{\widetilde{Y_{t}}}\left(2 E_{Y}\right)(-3)$. The conclusion then follows from the following easy vanishing statements:

$$
H^{1}\left(\widetilde{Y}_{t}, \mathcal{O}_{\widetilde{Y_{t}}}(-2)\right)=0 \quad \text { and } \quad H^{0}\left(\widetilde{Y}_{t}, \mathcal{O}_{\widetilde{Y_{t}}}(-2)\right)=0
$$


Remark 1.12. It is easy to check that $e_{Y}$ is in fact the class of the logarithmic tangent bundles exact sequence

$$
\left.0 \longrightarrow T_{\widetilde{Y_{t}}}\left(\log E_{Y}\right) \longrightarrow T_{\widetilde{\mathbb{P}^{4}}}\left(\log E_{\mathbb{P}^{4}}\right)\right|_{\widetilde{Y_{t}}} \longrightarrow \mathcal{O}_{\widetilde{Y_{t}}}(3)\left(-2 E_{Y}\right) \longrightarrow 0
$$

associated with the embedding of $\widetilde{Y}_{t}$ in the blow-up $\widetilde{\mathbb{P}^{4}}$ of the hyperplane $\mathbb{P}^{4}$ containing $Y_{t}$ at the singular point of $Y_{t}$.

(ii) This follows from the fact that $T_{\widetilde{Y_{t}}}\left(\log E_{Y}\right)$ is dual to $\Omega_{\widetilde{Y}_{t}}\left(\log E_{Y}\right)$ and that the later has determinant $K_{\widetilde{Y}_{t}}\left(E_{Y}\right)=\mathcal{O}_{\widetilde{Y_{t}}}(-2)\left(2 E_{Y}\right)$. Thus

$$
\Omega_{\widetilde{Y_{t}}}^{2}\left(\log E_{Y}\right) \cong T_{\widetilde{Y_{t}}}\left(\log E_{Y}\right)\left(K_{\widetilde{Y_{t}}}\left(E_{Y}\right)\right)=T_{\widetilde{Y_{t}}}\left(\log E_{Y}\right)(-2)\left(2 E_{Y}\right)
$$

(iii) The morphism $u: \mathcal{Y}_{U_{1}} \rightarrow U_{1}$ is smooth along the smooth locus $Y_{t, \text { reg }}$ of the fiber $Y_{t}$. It follows that the arguments used in the proof of Theorem 1.2 (ii) apply along $Y_{t, \text { reg }}$, so that we can conclude that the conclusion of (iii) holds true in $H^{1}\left(Y_{t, \text { reg }}, \Omega_{Y_{t, \mathrm{reg}}}^{2}\right)$, i.e., after composing with the restriction map $H^{1}\left(\widetilde{Y}_{t}, \Omega_{\widetilde{Y}_{t}}^{2}\left(\log E_{Y}\right)\right) \rightarrow H^{1}\left(Y_{t, \text { reg }}, \Omega_{Y_{t, \text { reg }}}^{2}\right)$. The proof is then finished using the following sublemma.

Sublemma 1.13. The restriction map $H^{1}\left(\widetilde{Y}_{t}, \Omega_{\widetilde{Y}_{t}}^{2}\left(\log E_{Y}\right)\right) \rightarrow H^{1}\left(Y_{t, \mathrm{reg}}, \Omega_{Y_{t, \mathrm{reg}}}^{2}\right)$ is an isomorphism.

Proof. Note that $Y_{t, \text { reg }}=\widetilde{Y}_{t} \backslash E_{Y}$. Denoting by $j: Y_{t, \text { reg }} \rightarrow \widetilde{Y}_{t}$ the inclusion map, $j$ is an affine map and we have $\Omega_{Y_{t, \mathrm{reg}}}^{2}=j^{*}\left(\Omega_{\widetilde{Y}_{t}}^{2}\left(\log E_{Y}\right)\right)$, so that

$$
H^{1}\left(Y_{t, \mathrm{reg}}, \Omega_{Y_{t, \mathrm{reg}}}^{2}\right)=H^{1}\left(\widetilde{Y}_{t}, R^{0} j_{*}\left(\left.\Omega_{\widetilde{Y}_{t}}^{2}\left(\log E_{Y}\right)\right|_{Y_{t, \mathrm{reg}}}\right)\right)=\underset{k}{\lim } H^{1}\left(\widetilde{Y}_{t}, \Omega_{\widetilde{Y}_{t}}^{2}\left(\log E_{Y}\right)\left(k E_{Y}\right)\right) .
$$

The lemma then follows from the following exact sequence:

$$
\left.0 \longrightarrow \Omega_{E_{Y}}^{2} \longrightarrow \Omega_{\widetilde{Y}_{t}}^{2}\left(\log E_{Y}\right)\right|_{E_{Y}} \longrightarrow \Omega_{E_{Y}} \longrightarrow 0 .
$$

Indeed, we recall that $E_{Y} \cong \mathbb{P}^{1} \times \mathbb{P}^{1}$ and that $\mathcal{O}_{E_{Y}}\left(E_{Y}\right)=\mathcal{O}_{E_{Y}}(-1,-1)$. It follows that, for any $k>0$,

$$
H^{1}\left(E_{Y}, \Omega_{E_{Y}}\left(k E_{Y}\right)\right)=0 \quad \text { and } \quad H^{1}\left(E_{Y}, \Omega_{E_{Y}}^{2}\left(k E_{Y}\right)\right)=0
$$

and, for any $k \geqslant 0$

$$
H^{0}\left(E_{Y}, \Omega_{E_{Y}}\left(k E_{Y}\right)\right)=0 \quad \text { and } \quad H^{0}\left(E_{Y}, \Omega_{E_{Y}}^{2}\left(k E_{Y}\right)\right)=0
$$

Using the exact sequence (1.19), this shows that

$$
H^{1}\left(E_{Y},\left.\Omega_{\widetilde{Y}_{t}}^{2}\left(\log E_{Y}\right)\right|_{E_{Y}}\left(k E_{Y}\right)\right)=0
$$


for $k>0$ and $H^{0}\left(E_{Y},\left.\Omega_{\widetilde{Y}_{t}}^{2}\left(\log E_{Y}\right)\right|_{E_{Y}}\left(k E_{Y}\right)\right)=0$ for $k \geqslant 0$. It follows that the map

$$
H^{1}\left(\widetilde{Y}_{t}, \Omega_{\widetilde{Y}_{t}}^{2}\left(\log E_{Y}\right)\left(k E_{Y}\right)\right) \longrightarrow H^{1}\left(Y_{t}, \Omega_{Y_{t}}^{2}\left(\log E_{Y}\right)\left((k+1) E_{Y}\right)\right)
$$

is an isomorphism for $k \geqslant 0$, proving the lemma by (1.18).

The proof of Lemma 1.11 is now complete.

Proof of Proposition 1.9. We have to prove that $\sigma_{U_{1}}$ is non-degenerate at any point of $\mathcal{J}_{U_{1}}^{\circ}$ over $t \in U_{1} \backslash U$. This is equivalent to proving that the map

$$
\lrcorner \sigma_{U_{1}}: T_{U_{1}, t} \longrightarrow H_{\mathrm{inv}}^{0}\left(\mathcal{J}_{U_{1}, t}^{\circ}, \Omega_{\mathcal{J}_{U_{1}, t}^{\circ}}\right)=H^{1}\left(\widetilde{Y}_{t}, \Omega_{\widetilde{Y}_{t}}^{2}\left(\log E_{Y}\right)\left(-E_{Y}\right)\right)
$$

is an isomorphism, where $H_{i n v}^{0}$ here denotes the space of translation-invariant 1-forms. Using Lemma 1.11, the last statement is equivalent to the fact that the multiplication map

$$
e_{Y}: H^{0}\left(\widetilde{Y}_{t}, \mathcal{O}_{\widetilde{Y_{t}}}(3)\right) \longrightarrow H^{1}\left(\widetilde{Y}_{t}, \Omega_{\widetilde{Y}_{t}}^{2}\left(\log E_{Y}\right)\left(-E_{Y}\right)\right)
$$

is an isomorphism, where $e_{Y} \in H^{1}\left(\widetilde{Y}_{t}, \Omega_{\widetilde{Y}_{t}}^{2}\left(\log E_{Y}\right)\left(-E_{Y}\right)(-1)\right)$. We have

$$
\Omega_{\widetilde{Y}_{t}}^{2}\left(\log E_{Y}\right)\left(-E_{Y}\right)(-1) \cong T_{\widetilde{Y_{t}}}\left(\log E_{Y}\right)(-3)\left(E_{Y}\right)
$$

and the class $e_{Y}$ maps to the extension class $e_{Y}^{\prime}$,

$$
\left.0 \longrightarrow T_{\widetilde{Y_{t}}}\left(\log E_{Y}\right) \longrightarrow T_{\widetilde{\mathbb{P}^{4}}}(\log E)\right|_{\widetilde{Y_{t}}} \longrightarrow \mathcal{O}_{\widetilde{Y_{t}}}\left(3-2 E_{Y}\right) \longrightarrow 0
$$

of the logarithmic normal bundle sequence of $\widetilde{\mathcal{Y}}_{t}$ in $\widetilde{\mathbb{P}^{4}}$, via the natural map

$$
H^{1}\left(\widetilde{Y}_{t}, T_{\widetilde{Y}_{t}}\left(\log E_{Y}\right)(-3)\left(E_{Y}\right)\right) \longrightarrow H^{1}\left(\widetilde{Y}_{t}, T_{\widetilde{Y}_{t}}\left(\log E_{Y}\right)(-3)\left(2 E_{Y}\right)\right)
$$

An element in the kernel of the multiplication map by $e_{Y}$ is thus also in

$$
\operatorname{Ker}\left(e_{Y}^{\prime}: H^{0}\left(\widetilde{Y}_{t}, \mathcal{O}_{\widetilde{Y}_{t}}(1)\right) \longrightarrow H^{1}\left(\widetilde{Y}_{t}, T_{\widetilde{Y}_{t}}\left(\log E_{Y}\right)(-2)\left(2 E_{Y}\right)\right)\right)
$$

induced by (1.21), hence comes from an element of

$$
H^{0}\left(\widetilde{Y}_{t},\left.T_{\widetilde{\mathbb{P}^{4}}}(\log E)\right|_{\widetilde{Y}_{t}}(-2)\left(2 E_{Y}\right)\right)
$$

and it is easily shown that this space reduces to zero. The map

$$
\lrcorner \sigma_{U_{1}}: T_{U_{1}, t} \longrightarrow H_{i n v}^{0}\left(\mathcal{J}_{U_{1}, t}^{\circ}, \Omega_{\mathcal{J}_{U_{1}, t}^{\circ}}\right)
$$

is thus injective, and hence an isomorphism. 


\section{Good and very good lines}

Our main tool for studying degenerations of intermediate Jacobians of cubic 3-folds is Mumford's description of the intermediate Jacobian $J(Y)$ as a Prym variety $\operatorname{Prym}(\widetilde{C} / C)$. The curve $C$ (and its étale double cover $\widetilde{C}$ ) are obtained by projecting from a generic line on the smooth cubic $Y$. In [17] (this is also subsequently used in [16]), it is noted that much of the Prym construction carries on to the mildly singular case provided a careful choice of a line $l$ on (the possibly singular) $Y$. This provides a powerful tool for studying the degenerations of intermediate Jacobians. We caution the reader that the context in the current paper is slightly different from that of [17], [16] (e.g. see Remark 2.4) forcing us to reprove (under slightly different hypotheses) and strengthen certain results. For convenience, we have tried to make the exposition below mostly self-contained.

Notation 2.1. If $X$ is a cubic 4-fold and $Y \subset X$ is a hyperplane section, we denote by $F(Y)$ (resp. $F(X)$, with $F(Y) \subset F(X))$ the varieties of lines in $Y$ (resp. $X$ ). We denote by $[l] \in F(Y)$ (resp. $F(X))$ the point parameterizing $l \subset Y$ (resp. $l \subset X)$.

\subsection{Good lines}

Definition 2.2. (Cf. [17, Definition 3.4]) Let $Y \subset \mathbb{P}^{4}$ be a cubic 3 -fold not containing any plane. A line $l \subset Y$ is good if for any plane $P \subset \mathbb{P}^{4}$ containing $l, P \cap Y$ consists in three distinct lines.

The notion of a good line is obviously important from the point of view of the Prym construction of the intermediate Jacobian of a cubic 3-fold. Projecting $Y$ from $l$, we get a conic bundle $\widetilde{Y}_{l} \rightarrow \mathbb{P}^{2}$, where $\widetilde{Y}_{l}$ is the blow-up of $Y$ along $l$, and the discriminant curve $C_{l} \subset \mathbb{P}^{2}$ parameterizing reducible conics has degree 5. The curve $\widetilde{C}_{l}$ of lines in $Y$ intersecting $l$ is the double cover of $C_{l}$ with fiber over the point $c$ parameterizing a reducible conic $C$ the set of components of $C$. Thus, if $l$ is good, the natural involution acting on $\widetilde{C}_{l}$ has no fixed point.

Proposition 2.3. Let $X$ be a general cubic 4-fold. Then any hyperplane section $Y$ of $X$ has a good line.

Remark 2.4. The existence of a good line is proved in [17] when $Y$ has singularities of type $A_{k}$ for $k \leqslant 5$, or $D_{4}$, i.e. the singularities relevant in the geometric invariant theory (GIT) context. Unfortunately, we need to allow some additional simple (or ADE) singularities (e.g. $D_{5}$ ) as these can appear as singularities of hyperplane sections of general cubic 4 -folds. It is very likely that the arguments of [17] could be extended to cover the cases needed in this paper, but we prefer to give an alternative proof here. 
Proof of Proposition 2.3. Let us say that a line $l$ in $X$ is special in $X$ (resp. in $Y$ ) if the restriction map $J_{X} \rightarrow H^{0}\left(l, \mathcal{O}_{l}(2)\right)$ (resp. $\left.J_{Y} \rightarrow H^{0}\left(l, \mathcal{O}_{l}(2)\right)\right)$ has rank $\leqslant 2$, where $J_{X}$ (resp. $J_{Y}$ ) denotes the degree-2 part of the Jacobian ideal of $X$ (resp. $Y$ ). As $X$ is general, lines which are special in $X$ are parameterized by a smooth surface $\Sigma_{\mathrm{sp}} \subset F(X)$ (see [6]). Recall from [64] that the variety $F(X)$ has a rational self-map $\phi: F(X) \rightarrow-F(X)$. The map $\phi$ associates with $[l] \in F(X)$ the point $\left[l^{\prime}\right]$ parameterizing the line $l^{\prime} \subset X$ constructed as follows: if $l$ is not special in $X$, that is $[l] \notin \Sigma_{\mathrm{sp}}$, there is a unique plane $P_{l} \subset \mathbb{P}^{5}$ such that $P_{l} \cap X=2 l+l^{\prime}$ as a divisor of $P_{l}$, where $l^{\prime} \subset X$ is the desired line in $X$. When $X$ contains no plane, the indeterminacy locus of $\phi$ is exactly the surface $\Sigma_{\mathrm{sp}}$, along which the plane $P_{l}$ above is not unique. Furthermore, the indeterminacies of the map $\phi$ are solved after blowing-up the surface $\Sigma_{\mathrm{sp}}$, and the induced morphism $\tilde{\phi}: \widetilde{F(X)} \rightarrow F(X)$ is finite if $X$ is general (see [6]). Note that the condition on a line $l^{\prime} \subset Y$ to being good will be implied by the slightly stronger fact that $l^{\prime}$ is non-special in $X$ (so $\phi$ is well defined at $\left[l^{\prime}\right]$ ) and for no point $[l] \in F(Y)$, one has $\tilde{\phi}([l])=\left[l^{\prime}\right]$ or $\phi\left(\left[l^{\prime}\right]\right)=[l]$. (For the first of these conditions, one has rather to consider a point over $[l]$ in $\widehat{F(X)}$.)

We first have the following lemma.

Lemma 2.5. (i) If $X$ is smooth, $F(Y)$ is a surface for any hyperplane section $Y$ of $X$. Furthermore, $F(Y)_{\text {red }} \subset F(X)$ is Lagrangian for the holomorphic 2-form $\sigma$ on the smooth hyper-Kähler manifold $F(X)$ (see [13]).

(ii) If $X$ is general, $F(Y)$ is irreducible and reduced for any hyperplane section of $X$.

Proof. (i) It is classical that $F(Y)$ is smooth of dimension 2 at any point $[l]$ parameterizing a line $l$ contained in the smooth locus of $Y$. Moreover, $Y$ has isolated singularities, and the families of lines in $Y$ through any point $y \in Y$ cannot be 3-dimensional as otherwise it would be the whole set of lines in $H_{Y}$ passing through $y$, where we denote by $H_{Y}$ the hyperplane cutting $Y$ in $X$. This proves the first statement. The $(2,0)$-form $\sigma$ on $F(X)$ is deduced from the class $\alpha$ generating $H^{3,1}(X)$ by the formula

$$
\sigma=P^{*} \alpha \quad \text { in } H^{2,0}(F(X))
$$

where $P \subset F(X) \times X$ is the incidence correspondence, so that $p: P \rightarrow F(X)$ is a $\mathbb{P}^{1}$-bundle over $F(X)$. Denoting by $P_{Y} \subset F(Y) \times Y$ the incidence correspondence of $Y$, we observe that, since $Y$ has only isolated singularities, $P_{Y}$ lifts to a correspondence $P_{\widetilde{Y}} \subset F(Y) \times \widetilde{Y}$, where $\widetilde{Y}$ is a desingularization of $Y$. If $U$ is any open set contained in the regular locus of $F(Y)_{\text {red }}$, we then have

$$
\left.\sigma\right|_{U}=\left.P^{*}(\alpha)\right|_{U}=P_{\widetilde{Y}}^{*}\left(j^{*} \alpha\right) \quad \text { in } H^{2,0}(U),
$$


where $j: \widetilde{Y} \rightarrow X$ is the desingularization map. Thus the vanishing of $\left.\sigma\right|_{U}$ follows from the vanishing of $j^{*} \sigma$ in $H^{3,1}(\widetilde{Y})$. To get the last vanishing, observe that $\widetilde{Y}$ is smooth of dimension 3 and rationally connected, so that we have $H^{2,0}(\widetilde{Y})=0$ hence also $H^{3,1}(\widetilde{Y})=0$. Thus $F(Y)_{\text {red }}$ is Lagrangian for $\sigma$.

(ii) The stated property is Zariski open, so it suffices to prove it when $X$ is very general. In this case, the space $\operatorname{Hdg}^{4}(F(X))$ of rational Hodge classes of degree 4 on $F(X)$ is of dimension 2. Let us say that a class $\gamma \in \operatorname{Hdg}^{4}(F(X))$ is Lagrangian if $\gamma \cup[\sigma]=0$ in $H^{6}(F(X), \mathbb{C})$. The class $l^{2}$, where $l$ is a Plücker hyperplane section of $F(X)$, is not Lagrangian by the second Hodge-Riemann bilinear relations and thus the space $\operatorname{Hdg}^{4}(F(X))_{\text {lag }}$ of Lagrangian rational Hodge classes on $F(X)$ is of dimension $\leqslant 1$. It follows that the class $[F(Y)] \in \operatorname{Hdg}^{4}(F(X))_{\text {lag }}$ cannot be written as the sum of two nonproportional Lagrangian classes. In fact, coming back to integer coefficients, it can neither be written as the sum of two proportional non-zero effective classes. Indeed, the class $[F(Y)] \in \operatorname{Hdg}^{4}(F(X), \mathbb{Z})$ is primitive, that is, not divisible by any non-zero integer $\neq \pm 1$, because when $X$ contains a plane $P, F(X)$ contains the dual plane $P^{*}$ and $[F(Y)] \cdot\left[P^{*}\right]=1$. We thus proved that $F(Y)$ is irreducible and reduced.

Coming back to the proof of Proposition 2.3, it is clear that for any hyperplane section $Y$ of $X$, there is a line contained in $Y$ which is non-special in $X$. Indeed, the surface $\Sigma_{\mathrm{sp}}$ of lines which are special in $X$ is irreducible and not contained in the surface of lines in $Y$ because it is smooth connected and not Lagrangian, see [6]; thus it can intersect $F(Y)$ only along a proper subset. Next, assume to the contrary that there is no good line in $Y$. This then means that for a general $[l] \in F(Y)$, (hence non-special for $X$ ), either (1) there is a $\left[l^{\prime}\right] \in F(Y)$ such that, for some plane $P \subset H_{Y}, P \cap Y=2 l+l^{\prime}$, that is $\phi(l)=l^{\prime}$, or $(2)$ there is a $\left[l^{\prime}\right] \in F(Y)$ such that for some plane $P \subset H_{Y}, P \cap Y=l+2 l^{\prime}$. In case (1), the map $\phi$ is well defined at the general point $[l] \in F(Y)$ hence of maximal rank at $[l]$ because $\phi^{*} \sigma=-2 \sigma$, hence $\phi(F(Y))=F(Y)$ by irreducibility of $F(Y)$. But then $\left[l^{\prime}\right]$ is also general in $F(Y)$, which implies that (2) occurs as well. So we just have to exclude (2). Note that the line $l^{\prime}$ is then special for $Y$.

There are two possibilities:

(a) The point $\left[l^{\prime}\right] \in F(Y)$ moves in a surface contained in $F(Y)$, and hence, by Lemma 2.5, every line in $Y$ is special for $Y$.

(b) The point $\left[l^{\prime}\right]$ moves in a curve $D \subset F(Y)$ and this curve is contained $\Sigma_{s p}$. Furthermore, for any $\left[l^{\prime}\right] \in D$, the 3 -dimensional projective space $Q_{l}=\bigcap_{x \in l} H_{X, x}$ is contained in $H_{Y}$, where $H_{X, x}$ denotes the hyperplane tangent to $X$ at $x$.

In case (a), we get a contradiction as follows: the general line $l^{\prime} \subset Y$ does not pass through a singular point of $Y$, and the fact that $l^{\prime}$ is special for $Y$ says exactly, by taking 
global sections in the normal bundle sequence

$$
0 \longrightarrow N_{l^{\prime} / Y}(-1) \longrightarrow N_{l^{\prime} / \mathbb{P}^{4}}(-1) \longrightarrow \mathcal{O}_{l^{\prime}}(2) \longrightarrow 0,
$$

that $H^{0}\left(N_{l^{\prime}} / Y(-1)\right) \neq 0$, hence that $N_{l^{\prime} / Y} \cong \mathcal{O}_{l^{\prime}}(1) \oplus \mathcal{O}_{l^{\prime}}(-1)$. But the fact that $N_{l^{\prime}} / Y$ has this form says equivalently that the map $q: P_{Y} \rightarrow Y$ is not submersive at any point of the fiber $P_{Y,\left[l^{\prime}\right]} \subset P_{Y}$ of $p: P_{Y} \rightarrow F(Y)$ over the point $\left[l^{\prime}\right]$. As $q\left(P_{Y}\right)=Y$, this contradicts the fact that $\left[l^{\prime}\right]$ is general in $F(Y)$.

The case (b) is excluded by the following lemma which we will use again later.

Lemma 2.6. Let $X$ be a general cubic 4-fold. Then every hyperplane section $Y \subset X$ contains only finitely many cubic surfaces which are singular along a line.

Proof. Assume to the contrary that there is a curve $D$ of such surfaces and such lines $l^{\prime}$ of singularities. We note that every line $l^{\prime}$ parameterized by a point $\left[l^{\prime}\right] \in D$ has to pass through a singular point of $Y$. Indeed, if $Y$ is smooth along $l^{\prime}$, then its Gauss map given by the partial derivatives of the defining equation $f_{Y}$ of $Y$ in $H_{Y}$ is well defined along $l^{\prime}$, and thus it cannot be constant along $l$, hence equivalently $Y \cap \mathbb{P}^{3}$ cannot be singular at all points of $l^{\prime}$ for any $\mathbb{P}^{3}$ containing $l$. Next, a hyperplane section $Y$ of $X$ has finitely many singular points, and hence we may assume that, in case (b), the curve $D$ consists of lines passing through a given singular point $y$ of $Y$. By Lemma 2.5, the family $C_{y}$ of lines in $X$ passing through $y$ is a curve, and thus $D$ must be an irreducible component of $C_{y}$. In adequate homogeneous coordinates $X_{0}, \ldots, X_{4}$ on $H_{Y}$, the point $y$ has equations $X_{i}=0$, $i=0, \ldots, 3$, and $Y$ has equation $X_{4} Q\left(X_{0}, \ldots, X_{3}\right)+T\left(X_{0}, \ldots, X_{3}\right)=0$, where $Q$ and $T$ are homogeneous polynomials of degrees 2 and 3 , respectively. The curve $C_{y}$ of lines through $y$ (in $Y$ or $X$ ) is defined by the equations $Q=T=0$. Let $\left[l^{\prime}\right] \in C_{y}$ parameterize a line $l^{\prime}$ in $Y$ such that some hyperplane $H^{\prime}$ in $H_{Y}$ containing $l^{\prime}$ is tangent to $Y$ everywhere along $l^{\prime}$. This is saying that the equation $f:=X_{4} Q\left(X_{0}, \ldots, X_{3}\right)+T\left(X_{0}, \ldots, X_{3}\right)=0$, restricted to a hyperplane $H^{\prime}$ of $\mathbb{P}^{3}$ passing through the point $\left[l^{\prime}\right]$, has zero derivatives along $l^{\prime}$. Thus the equations $Q$ and $T$ restricted to $H^{\prime}$, must have zero derivative at $\left[l^{\prime}\right]$. It follows that the two polynomials $Q$ and $T$ have non-independent derivatives at $\left[l^{\prime}\right]$, so that $\left[l^{\prime}\right]$ is a singular point of the curve $C_{y}$. In conclusion, we found that, under our assumption, the curve $C_{y}$ has a non-reduced component. Hence, the proof of Lemma 2.6 is concluded by the proof of Lemma 2.7 below.

Lemma 2.7. If $X$ is general, the curve $C_{y}$ of lines through any point $y \in X$ is reduced.

Remark 2.8. It is not true that $C_{y}$ is irreducible for any $y$. Indeed, a general $X$ contains a cubic surface which is a cone over an elliptic curve, with vertex $y \in X$. Hence, the elliptic curve is an irreducible component of $C_{y}$ in this case. 
Proof of Lemma 2.7. For any $y \in X$, the curve $C_{y}$ has degree 6, and the cone over the curve $C_{y}$, with vertex $y$, is a degree- 6 surface contained in $X$. We now use the fact that $X$ is very general, and hence $\operatorname{Hdg}^{4}(X, \mathbb{Z})=\mathbb{Z} h^{2}$, where $h=c_{1}\left(\mathcal{O}_{X}(1)\right)$. Therefore any surface in $X$ has degree divisible by 3. Applying this to the components of this cone, the only way the curve $C_{y}$ can be non-reduced is if $C_{y}$ is everywhere non-reduced with multiplicity 2. The curve $C_{y \text {,red }}$ is then a curve of degree 3 which can be either a plane cubic or a normal rational curve of degree 3 . If $S$ is the cone over a normal cubic curve in $\mathbb{P}^{3}$, the set of cubic hypersurfaces in $\mathbb{P}^{5}$ containing $S$ has codimension 22 , while the dimension of the Hilbert scheme parameterizing such an $S$ in $\mathbb{P}^{5}$ is $5+4+15-3=21$, so that a general cubic does not contain such a surface. In the case of the cone over an irreducible plane cubic, the curve $C_{y}$ is the complete intersection of a quadric and a cubic in $\mathbb{P}^{3}$ which contains an irreducible plane cubic with multiplicity 2 . The only possibility is then that the quadric itself is a double plane. However, one can easily check that, for general $X$, there is no point $y \in X$ where the Hessian of the defining equation of $X$ defines a non-reduced quadric in $\mathbb{P}^{3}$.

The proof of Proposition 2.3 is now complete.

\subsection{Existence of very good lines}

For constructing compactified Jacobian fibrations (and similarly compactified Prym fibrations) irreducibility assumptions are crucial. This leads us to the following strengthening of the notion of good line.

Definition 2.9. Let $Y$ be a cubic 3-fold. We will say that a line $l \subset Y$ is very good if $l$ is good (see Definition 2.2) and the curve $\widetilde{C}_{l}=\widetilde{C}_{l, Y}=\{$ lines in $Y$ meeting $l\}$ is irreducible.

Proposition 2.10. Let $X$ be a general cubic 4-fold. Then, for any hyperplane section $Y \subset X$, there exists a line $l \subset Y$ such that the curve $\widetilde{C}_{l, Y}$ is irreducible.

Corollary 2.11. If $X$ and $Y$ are as above, a general line in $Y$ is very good.

Proof. Proposition 2.3 shows the existence of a good line, and this is an open property on $F(Y)$. Proposition 2.10 shows the existence of a line $l \subset Y$ such that the curve $\widetilde{C}_{l, Y}$ is irreducible, and this is also an open property on $F(Y)$. As we know by Lemma 2.5 that $F(Y)$ is irreducible, it follows that a general line is very good.

Proof of Proposition 2.10. We consider the incidence variety $P_{Y} \subset F(Y) \times Y$, which is a $\mathbb{P}^{1}$-bundle $p: P_{Y} \rightarrow F(Y)$ over $Y$. We proved in Lemma 2.5 that, being $X$ and $Y$ as above, $F(Y)$ is irreducible and reduced, and thus $P_{Y}$ satisfies the same properties. In 
particular, the degree of the map $q: P_{Y} \rightarrow Y$ is 6 as for a smooth $Y$, and the degree of the map $q^{\prime}=p r_{2}:\left(P_{Y} \times_{Y} P_{Y}\right) \backslash \Delta_{P_{Y}} \rightarrow P_{Y}$ is 5 . We have the following lemma.

Lemma 2.12. Let $X$ be a general cubic 4-fold and let $Y$ be any hyperplane section of $X$. If for all lines $l \subset Y$ the curve $\widetilde{C}_{l}$ is reducible, then $\left(P_{Y} \times_{Y} P_{Y}\right) \backslash \Delta_{P_{Y}}$ has at least two irreducible components dominating $Y$.

Proof. We observe first that for any line $l \subset Y$ the curve $\widetilde{C}_{l} \subset F(Y)$ (minus the point $[l]$ when $l$ is special for $Y$ ) identifies naturally with $q^{-1}(l)$ away from its intersection with the vertical curve $P_{Y,[l]}=p^{-1}([l])$. Indeed $q^{-1}(l)$ is, away from the vertical fiber $P_{Y,[l]}$, the set of pairs $\left(\left[l^{\prime}\right], x\right)$ such that $[l] \neq\left[l^{\prime}\right]$ and $x \in l \cap l^{\prime}$. The curve $\widetilde{C}_{l}$ (away from $[l]$ when $l$ is special) thus maps to it via the map

$$
\left[l^{\prime}\right] \longmapsto\left(\left[l^{\prime}\right], x\right), \quad \text { with }\{x\}=l \cap l^{\prime}
$$

We will in fact see $\widetilde{C}_{l}$ (minus the point [l]) as contained in $\left(P_{Y} \times_{Y} P_{Y}\right) \backslash \Delta_{P_{Y}}$ by the map

$$
\left[l^{\prime}\right] \longmapsto\left(\left[l^{\prime}\right],[l], x\right), \quad \text { with }\{x\}=l \cap l^{\prime}
$$

Now suppose that $\widetilde{C}_{l}$ is reducible for all $l$. For general $l, \widetilde{C}_{l}$ is reduced and the morphism $q_{l}^{\prime}=\left.q^{\prime}\right|_{\widetilde{C}_{l}}: \widetilde{C}_{l} \rightarrow l$ has degree 5 . So, for general $l$, we must be in one of the following situations:

(1) $\widetilde{C}_{l}$ has no component of degree 1 over $l$ and has a unique component $\widetilde{C}_{l, 2}$ of degree 2 over $l$. We then denote by $\widetilde{C}_{l, 3}$ the Zariski closure of $\widetilde{C}_{l} \backslash \widetilde{C}_{l, 2}$.

(2) $\widetilde{C}_{l}$ has $1 \leqslant k<4$ components $\widetilde{C}_{l, 1, i}$ of degree 1 over $l$ and the rest

$$
\widetilde{C}_{l, \mathrm{rem}}=\overline{\widetilde{C}_{l} \backslash \bigcup_{i=1}^{k} \widetilde{C}_{l, 1, i}}
$$

has all its components of degree $>1$ over $\widetilde{C}_{l}$.

(3) $\widetilde{C}_{l}$ has five components of degree 1 over $l$.

If case (1) or case (2) happens, then $\left(P_{Y} \times_{Y} P_{Y}\right) \backslash \Delta_{P_{Y}}$ has at least two irreducible components dominating $P_{Y}$, namely the two varieties $\bigcup_{[l] \in F(Y)} \widetilde{C}_{l, 2}$ and $\bigcup_{[l] \in F(Y)} \widetilde{C}_{l, 3}$ in case $(1)$, and the two varieties $\bigcup_{[l] \in F(Y)} \bigcup_{i=1}^{k} \widetilde{C}_{l, 1, i}$ and $\bigcup_{[l] \in F(Y)} \widetilde{C}_{l, \text { rem }}$ in case (2). It thus suffices to show that (3) cannot happen. This however follows from Proposition 2.3. Indeed, we may assume that $l$ is good, so that the involution on $\widetilde{C}_{l}$ has no fixed point. But if $\widetilde{C}_{l}$ has five irreducible components all isomorphic to $\mathbb{P}^{1}$, one of them is fixed under the involution which then has fixed points.

Proposition 2.10 now follows from Lemma 2.12 and from the following one. 
Lemma 2.13. If $X$ is general, then, for any hyperplane section $Y$ of $X$, the variety $\left(P_{Y} \times_{Y} P_{Y}\right) \backslash \Delta_{P_{Y}}$ is irreducible.

Let us prove a few intermediate statements.

LEMMA 2.14. If $X$ is general, and $Y$ is any hyperplane section of $X$, any irreducible component of $\left(P_{Y} \times_{Y} P_{Y}\right) \backslash \Delta_{P_{Y}}$ dominates $P_{Y}$ by the second projection.

Proof. The only possibility for an irreducible component $Z$ of $P_{Y} \times{ }_{Y} P_{Y}$ not to dominate $P_{Y}$ by the second projection is if there is a curve $W \subset Y$ such that, for any $y \in W$, there is a curve $D_{y}$ of lines in $Y$ passing through $y$. However, this is impossible by the following claim.

Claim 2.15. For general $X$, and for any hyperplane section $Y$ of $X$, there are only finitely many points $y \in Y$ such that the set of lines in $Y$ through $y$ contains a curve $D_{y}$.

Proof. The stated property of $X$ is Zariski open, so it suffices to prove it assuming that $X$ is very general. Assume by contradiction that this set is a curve $W$. Then the union over $y \in W$ of the $D_{y}$ 's would then be a surface contained in $F(Y)$, and since we know by Lemma 2.5 that $F(Y)$ is irreducible, this surface would be the whole of $F(Y)$. Thus $Y$ has the property that any line in $Y$ meets the curve $W \subset Y$. But the general point $[l] \in F(Y)$ is a smooth point of $F(Y)$ parameterizing a line $l$ with normal bundle $N_{l / X} \cong \mathcal{O}_{l} \oplus \mathcal{O}_{l}$, which means that the morphism $q: P_{Y} \rightarrow Y$ is étale in a neighborhood of the fiber $P_{Y,[l]}$ of $P_{Y}$ over $[l] \in F(Y)$, so that the general deformation of $l$ in $Y$ does not intersect $Z$.

Lemma 2.14 is thus proved.

The variety $\left(P_{Y} \times_{Y} P_{Y}\right) \backslash \Delta_{P_{Y}}$ has several rational involutions. We denote by $\tau$ the involution exchanging factors and by $i$ the involution of $\left(P_{Y} \times_{Y} P_{Y}\right) \backslash \Delta_{P_{Y}}$ which maps $\left(l_{1}, l_{2}\right), l_{1} \cap l_{2} \neq \varnothing$, to $\left(l_{3}, l_{2}\right)$, where $l_{3}$ is the residual line of the intersection $P_{l_{1}, l_{2}} \cap Y$, with $P_{l_{1}, l_{2}}$ being the plane generated by $l_{1}$ and $l_{2}$. Recall from (2.1) that the fiber of the composite map

$$
P_{Y} \times_{Y} P_{Y} \stackrel{\mathrm{pr}_{2}}{\longrightarrow} P_{Y} \stackrel{p}{\longrightarrow} F(Y)
$$

over $[l] \in F(Y)$ identifies with the curve $\widetilde{C}_{l}$ of lines in $Y$ meeting $l$, and that $i$ acts on $\widetilde{C}_{l}$ as the Prym involution. The quotient $\widetilde{C}_{l} / i$ is the discriminant curve $C_{l}$ of the conic bundle $\pi_{l}: \widetilde{Y}_{l} \rightarrow \mathbb{P}^{2}$.

Lemma 2.16. If $X$ is general and $Y$ is any hyperplane section of $X$, then the quotient

$$
\frac{\left(P_{Y} \times_{Y} P_{Y}\right) \backslash \Delta_{P_{Y}}}{i}
$$

is irreducible. 
Proof. Using Lemma 2.14, it suffices to show that, being $X$ and $Y$ as above, for general $[l] \in F(Y)$ the curve $C_{l}$ is irreducible. The curve $C_{l}$ is a quintic curve, so if it is not irreducible, it must decompose either as

(a) the union of a line and a quartic, or

(b) the union of a smooth conic and a cubic.

Case (a) is excluded as follows. A line component in the discriminant curve provides a cubic surface $S=P_{3} \cap Y$, where $P_{3}$ is a $\mathbb{P}^{3}$ contained in $H_{Y}$, which contains a 1-parameter family of lines meeting $l$. The surface $S$ is irreducible because $X$ is general, so does not contain a plane or a quadric surface. Furthermore $S$ cannot be a cone over an elliptic curve, because $l$ is general, and hence, by Claim 2.15, through any point $y$ of $l$ there are only finitely many lines in $Y$ through $y$. By assumption, for any plane $P \subset P_{3}$ containing $l$, the intersection $P \cap S$ contains $l$ and a residual reducible conic. The singular point of the conic moves in $S$, and hence, by Bertini, the singular locus of $S$ consists of a curve $\bar{Z}$ and thus must be a line $l^{\prime}$. Indeed, any bisecant line to $\bar{Z} \subset \operatorname{Sing} S$ is contained in $S$, and the only alternative possibility would be that $\bar{Z}$ is a conic and $S$ has a component which is a plane which is excluded, since $X$ is general. The line $l^{\prime}$ is then a special line of $X$ whose associated $\mathbb{P}_{l^{\prime}}^{3}=\bigcap_{x \in l^{\prime}} T_{X, x}$ is equal to $P_{3}$. We know by Lemma 2.6 that there are finitely many such $\mathbb{P}_{l^{\prime}}^{3}$ contained in $H_{Y}$ (or equivalently, cubic surfaces singular along a line $l^{\prime}$ and contained in $Y$ ), so the general line $l$ in $Y$ cannot be contained in such a $\mathbb{P}_{l^{\prime}}^{3}$.

Case (b) is excluded as follows. Suppose that the discriminant curve $C_{l}$ has a component which is a smooth conic $C$. As $l$ is a good line, the double cover $r: \widetilde{C}_{l} \rightarrow C_{l}$ is étale, hence split over $C: r^{-1}(C)=C_{1} \cup C_{2}$. Let $\pi_{l}: \widetilde{Y}_{l} \rightarrow \mathbb{P}^{2}$ be the linear projection from $l$, and let $T:=\pi_{l}^{-1}(C)$. Then $T$ is a reducible surface, $T=T_{1} \cup T_{2}$, where $T_{1}$ is swept-out by lines in $C_{1}$ and $T_{2}$ is swept-out by lines in $C_{2}$. On the other hand, as $X$ is very general, any surface in $X$ has degree divisible 3 , thus $T_{1}$ and $T_{2}$ must have degree 3 . The surfaces $T_{1}$ and $T_{2}$ are ruled surfaces using their 1-parameter family of lines intersecting $l$, and none of them can be contained in a projective subspace $P_{3} \subset H_{Y}$, since otherwise $P_{3}$ would contain $l$, and thus would project via $\pi_{l}$ to a line in $\mathbb{P}^{2}$, while the image $\pi_{l}\left(T_{i}\right)$ is our smooth conic.

Finally, a ruled non-degenerate degree- 3 surface in $\mathbb{P}^{4}$ is a cone over a rational normal curve or a projection of a Veronese surface from one of its points. The first case corresponds to the vector bundle $\mathcal{O} \oplus \mathcal{O}(3)$ on $\mathbb{P}^{1}$, while the second one corresponds to the vector bundle $\mathcal{O}(1) \oplus \mathcal{O}(2)$ on $\mathbb{P}^{1}$. We already explained by counting parameters that the general cubic hypersurface $X$ in $\mathbb{P}^{5}$ does not contain a cone over a rational cubic curve in $\mathbb{P}^{3}$. It is also true that the general cubic hypersurface $X$ in $\mathbb{P}^{5}$ does not contain the projection of a Veronese surface from one of its point, but this does not follow from an immediate dimension count. One has to argue as follows: this surface $V$ is smooth with 
$c_{1}(V)^{2}=8$ and $c_{2}(V)=4$. If $V \subset X$, the normal bundle $N_{V / X}$ fits into the exact sequence

$$
\left.0 \longrightarrow T_{V} \longrightarrow T_{X}\right|_{V} \longrightarrow N_{V / X} \longrightarrow 0
$$

As

$$
c_{2}\left(T_{X}\right)=6 h^{2} \quad \text { and } \quad c_{1}\left(T_{X}\right)=3 h,
$$

one gets from $(2.2)$ the following equalities in $H^{*}(V, \mathbb{Q})$ :

$$
\begin{aligned}
c_{1}\left(N_{V / X}\right) & =-c_{1}\left(T_{V}\right)+3 h_{V}, c_{2}\left(N_{V / X}\right)=-c_{2}\left(T_{V}\right)-c_{1}\left(N_{V / X}\right) \cdot c_{1}\left(T_{V}\right)+6 h_{V}^{2} \\
& =-c_{2}\left(T_{V}\right)-\left(-c_{1}\left(T_{V}\right)+3 h_{V}\right) \cdot c_{1}\left(T_{V}\right)+6 h_{V}^{2},
\end{aligned}
$$

where $h_{V}=c_{1}\left(\mathcal{O}_{V}(1)\right)$ and $h_{V}^{2}=3$. In the ruled surface $T=\mathbb{P}\left(\mathcal{O}_{\mathbb{P}^{1}}(1) \oplus \mathcal{O}_{\mathbb{P}^{1}}(2)\right) \stackrel{\pi}{\longrightarrow} \mathbb{P}^{1}$, one has

$$
K_{V}=-c_{1}\left(T_{V}\right)=-2 h_{V}+\pi^{*} \mathcal{O}_{\mathbb{P}^{1}}(3),
$$

which, combined with (2.3), gives

$$
c_{2}\left(N_{V / X}\right)=-4-\left(-2 h_{V}+\pi^{*} c_{1}\left(\mathcal{O}_{\mathbb{P}^{1}}(3)\right)+3 h_{V}\right) \cdot\left(2 h_{V}-\pi^{*} c_{1}\left(\mathcal{O}_{\mathbb{P}^{1}}(3)\right)\right)+18=5 .
$$

This shows that the self-intersection of $V$ in $X$ is equal to 5 , so that the class of $V$ is not equal to $h^{2}$. Hence such a surface does not exist for general $X$.

We get the following corollary (where again $X$ is general and $Y$ is any hyperplane section of $X)$.

Corollary 2.17. (i) The fibered product $\left(P_{Y} \times_{Y} P_{Y}\right) \backslash \Delta_{P_{Y}}$ has at most two irreducible components and, if $\left(P_{Y} \times_{Y} P_{Y}\right) \backslash \Delta_{P_{Y}}$ is reducible, the two components are exchanged by the rational involution $i$ acting on each curve $\widetilde{C}_{l} \times[l] \subset P_{Y} \times_{Y} P_{Y}$.

(ii) Let $\tau$ be the involution of $\left(P_{Y} \times_{Y} P_{Y}\right) \backslash \Delta_{P_{Y}}$ which exchanges the factors. If $\left(P_{Y} \times_{Y} P_{Y}\right) \backslash \Delta_{P_{Y}}$ is reducible, then its two components are exchanged by $\tau$.

Proof. (i) is an immediate consequence of Lemmas 2.16 and 2.14. The proof of (ii) goes as follows: We observe that the two rational involutions $\sigma$ and $\tau$ are part of an action of the symmetric group $\mathfrak{S}_{3}$ on $\left(P_{Y} \times_{Y} P_{Y}\right) \backslash \Delta_{P_{Y}}$ by birational maps. Indeed, $\left(P_{Y} \times_{Y}\right.$ $\left.P_{Y}\right) \backslash \Delta_{P_{Y}}$ can also be seen as the set of labelled triangles, that is, triples $\left(l_{1}, l_{2}, l_{3}\right)$ of lines in $Y$ such that, for some plane $P \subset H_{Y}, P \cap Y=l_{1}+l_{2}+l_{3}$. The action of $\mathfrak{S}_{3}$ is simply the permutation of the labels. Note that these birational maps are well defined at each generic point of $\left(P_{Y} \times_{Y} P_{Y}\right) \backslash \Delta_{P_{Y}}$ by Lemma 2.14. Note that $i$ is the involution $\left(l_{1}, l_{2}\right) \mapsto\left(l_{3}, l_{2}\right)$, while $\tau$ is the involution $\left(l_{1}, l_{2}\right) \mapsto\left(l_{2}, l_{1}\right)$. In any case, these two involutions are conjugate in $\mathfrak{S}_{3}$. We know by (i) that, if there are two irreducible components, then they are exchanged by $i$. Hence they must be also exchanged by $\tau$. 
Proof of Lemma 2.13. Let $X$ be a general cubic 4 -fold and let $Y$ be any hyperplane section of $X$. Assume by contradiction that $\left(P_{Y} \times_{Y} P_{Y}\right) \backslash \Delta_{P_{Y}}$ is not irreducible. Then, by Corollary 2.17 , it has exactly two components $\mathcal{C}_{1}$ and $\mathcal{C}_{2}$. Both components dominate $Y$, by Lemma 2.14. Let $k_{1}$ and $k_{2}$, with $1 \leqslant k_{1}<k_{2}$ and $k_{1}+k_{2}=5$, be the respective degrees of $\operatorname{pr}_{2}: \mathcal{C}_{1} \rightarrow P_{Y}$ and $\operatorname{pr}_{2}: \mathcal{C}_{2} \rightarrow P_{Y}$. One has $\left(k_{1}, k_{2}\right)=(2,3)$ or $\left(k_{1}, k_{2}\right)=(1,4)$. For a general point $y \in Y$, denote by $\left\{l_{1}, \ldots, l_{6}\right\}=q^{-1}(y)=: E_{y} \subset P_{Y}$. For $l_{i} \neq l_{j}$, we have $\left(l_{i}, l_{j}\right) \in$ $\left(P_{Y} \times_{Y} P_{Y}\right) \backslash \Delta_{P_{Y}}$, and thus we can write $\left(E_{y} \times E_{y}\right) \backslash \Delta_{E_{y}}$ as a disjoint union

$$
\left(E_{y} \times E_{y}\right) \backslash \Delta_{E_{y}}=E_{1, y} \sqcup E_{2, y},
$$

where

$$
E_{1, y}:=\left(E_{y} \times E_{y} \backslash \Delta_{E_{y}}\right) \cap \mathcal{C}_{1} \quad \text { and } \quad E_{2, y}:=\left(E_{y} \times E_{y} \backslash \Delta_{E_{y}}\right) \cap \mathcal{C}_{2} .
$$

The partition (2.4) satisfies the following properties:

(a) for any $i \in\{1, \ldots, 6\}$, the set of $j \neq i$ such that $\left(l_{i}, l_{j}\right) \in E_{1, y}$ has cardinality $k_{1}$, while the set of $j \neq i$ such that $\left(l_{i}, l_{j}\right) \in E_{2, y}$ has cardinality $k_{2}$;

(b) for any $i, j \in\{1, \ldots, 6\}$ with $i \neq j$, one has $\left(l_{i}, l_{j}\right) \in E_{1, y}$ if and only if $\left(l_{j}, l_{i}\right) \in E_{2, y}$.

Indeed, (b) is exactly Corollary 2.17 (ii).

The contradiction is now obvious: Indeed, (a) shows that the cardinality of $E_{1, y}$ is $6 k_{1}$ and the cardinality of $E_{2, y}$ is $6 k_{2}$, with $6 k_{1} \neq 6 k_{2}$, while (b) implies that the two sets have the same cardinality.

Proposition 2.10 is thus proved.

\section{Transversality arguments}

This section is devoted to applying transversality arguments in order to deduce that some statements which hold in large codimension for cubic 3-folds in $\mathbb{P}^{4}$ hold for every hyperplane sections of a general cubic 4-fold. In particular, we will first prove Lemma 3.8 which guarantees that the versality statement of [17] is actually satisfied by the family of quintic curves associated with the family of hyperplane sections of a general cubic 4-fold and a local choice of good lines in them. This will be needed in $\S 4$. In $\S 3.2$, we will extend this result to the case of a general Pfaffian cubic 4-fold. By applying a similar transversality argument, we will also prove the existence of a very good line in every hyperplane section of a general Pfaffian cubic 4-fold. This will be needed in order to make the arguments of $\S 4$ apply as well when the cubic 4 -fold is a general Pfaffian cubic (see $\S 6$ ). 


\subsection{Transversality results for general cubic 4-folds}

Below, we denote by $\mathcal{H y p}{ }_{4,3}^{0}$ the open subset of $\mathbb{P}\left(H^{0}\left(\mathbb{P}^{5}, \mathcal{O}_{\mathbb{P}^{5}}(3)\right)\right)$ parameterizing smooth cubic 4 -folds, and by $\mathcal{H} \mathrm{yp}_{3,3}$ the projective space $\mathbb{P}\left(H^{0}\left(\mathbb{P}^{4}, \mathcal{O}_{\mathbb{P}^{4}}(3)\right)\right)$. By restriction from $\mathbb{P}^{5}$ to a given $\mathbb{P}^{4} \subset \mathbb{P}^{5}$, we get a morphism

$$
r: \mathcal{H y p}_{4,3}^{0} \longrightarrow \mathcal{H y p}_{3,3},
$$

which is obviously smooth, since the fibers are Zariski open subsets of $H^{0}\left(\mathbb{P}^{5}, \mathcal{O}_{\mathbb{P}^{5}}(2)\right)$. With these notations, the following transversality lemma holds.

Lemma 3.1. (i) Let $Z \subset \mathcal{H} \mathrm{yp}_{3,3}$ be a closed algebraic subset of codimension $\geqslant 6$, which is invariant under the action of $\mathrm{PGL}(5)$. Then for a general $[X] \in \mathcal{H}_{\mathrm{yp}}{ }_{4,3}$, no hyperplane section of $X$ is isomorphic to a cubic 3-fold $Y$ parameterized by a point of $Z$.

(ii) Let $\mathcal{M}^{\prime} \subset \mathcal{H y p}{ }_{4,3}^{0}$ be a hypersurface which is invariant under PGL(5), and let $Z \subset \mathcal{H y p}{ }_{3,3}$ be a closed algebraic subset of codimension $\geqslant 7$, which is invariant under the action of $\mathrm{PGL}(5)$. Then, for a general $[X] \in \mathcal{M}^{\prime}$, no hyperplane section of $X$ is isomorphic to a cubic 3-fold $Y$ parameterized by a point of $Z$.

Proof. (i) Indeed, as $r$ is smooth (actually, flat would suffice), $r^{-1}(Z) \subset \mathcal{H y p}{ }_{4,3}^{0}$ has codimension 6 in $\mathcal{H y p}_{4,3}^{0}$. The group $\operatorname{PGL}(6,5) \subset \operatorname{PGL}(6)$ of automorphisms of $\mathbb{P}^{5}$ preserving $\mathbb{P}^{4}$ acts on $\mathcal{H y p} p_{4,3}^{0}$ preserving $r^{-1}(Z)$, since $Z$ is invariant under PGL(5). It thus follows that

$$
\operatorname{dim} \operatorname{PGL}(6) \cdot r^{-1}(Z) \leqslant 5+\operatorname{dim} r^{-1}(Z),
$$

or equivalently codim $\operatorname{PGL}(6) \cdot r^{-1}(Z) \geqslant 1$. Thus $\operatorname{PGL}(6) \cdot r^{-1}(Z)$ is not open in $\mathcal{H y p}_{4,3}^{0}$, which proves (i).

(ii) The same argument as in (1) shows that $\operatorname{PGL}(6) \cdot r^{-1}(Z)$ has codimension at least 2 in $\mathcal{H y p}_{4,3}^{0}$, and hence cannot contain the hypersurface $\mathcal{M}^{\prime}$.

Remark 3.2. We will see in $§ 3.2$ an improved version of Lemma 3.1 (ii), where under a certain assumption on the hypersurface $\mathcal{M}^{\prime}$, the estimate on codimension of $Z$ will be also 6, not 7. The hypersurface of interest for us will be the locus of Pfaffian cubics.

The above lemma will allow us to exclude from our study highly singular cubic 3folds and to restrict ourselves to mildly singular cubic 3 -folds with the following precise meaning.

Definition 3.3. Let $Y$ be a cubic 3-fold. We say $Y$ is allowable (or mildly singular) if $Y$ has at worst isolated singularities and $\tau_{\text {tot }}(Y) \leqslant 6$. Here $\tau$ denotes the Tjurina number of an isolated hypersurface singularity, and $\tau_{\text {tot }}(Y)$ is the sum of the associated Tjurina numbers, i.e. $\tau_{\text {tot }}(Y)=\sum_{p \in \operatorname{Sing}(Y)} \tau\left(Y_{p}\right)$ (where $Y_{p}$ denotes the germ of $Y$ at $p$ ). 
Remark 3.4. We recall that, for an isolated hypersurface singularity $(V(f), 0) \subset \mathbb{C}^{n}$, the Tjurina number is defined to be

$$
\tau(f)=\operatorname{dim}_{\mathbb{C}} \frac{\mathbb{C}\left[x_{1}, \ldots, x_{n}\right]}{\left\langle f, \partial f / \partial x_{1}, \ldots, \partial f / \partial x_{n}\right\rangle},
$$

and is the expected codimension in moduli to encounter that singularity. By dimension count, we thus expect that all hyperplane sections $Y$ of a general cubic 4-fold $X$ are allowable in the sense of Definition 3.3. The results below say that this is indeed the case.

Remark 3.5. The arguments involving good lines are closely related and inspired by those in [17]. However, as already noted in Remark 2.4, the results of [17] do not suffice here. Namely, in [17] the focus was on GIT stable/semi-stable cubic 3-folds, while here we focus on hyperplane sections of general cubic 4-folds (or general Pfaffian cubics). Thus, our notion of allowable is slightly different from that of [17, Definition 2.2].

Proposition 3.6. Let $Y$ be an allowable cubic 3-fold in the sense of Definition 3.3. Then, the following statements hold:

(0) $Y$ has at worst ADE (in particular planar) singularities.

(1) The deformations of $Y$ in $\mathbb{P}^{4}$ induce a simultaneous versal deformation of the singularities of $Y$. This means equivalently that the natural map from the first-order deformation space of $Y$, that is $H^{0}\left(Y, \mathcal{O}_{Y}(3)\right)$, to the product $\prod_{p \in \operatorname{Sing} Y} T_{Y_{p}}^{1}$, where $T_{Y_{p}}^{1}$ classifies the first-order deformations of the germ of singularities of $Y$ at $p$, is surjective.

(2) Assume additionally that there exists a good line $l \subset Y$. Let $\widetilde{C}_{l}$ be the curve of lines in $Y$ meeting $l$. Then, the singular points of $Y$ are in bijection with the singular points of the curve $C_{l}=\widetilde{C}_{l} / \iota$ (which is a plane quintic curve), the analytic types of corresponding singularities of $Y$ and $C_{l}$ coincide and the deformation theory of corresponding singular points of $Y$ and $C_{l}$ coincide. Furthermore, the deformations of $C_{l}$ give simultaneous versal deformations of the singularities of $C_{l}$ (which is compatible with the deformations of the singularities of $Y$ ).

(3) The locus of cubic hypersurfaces $Y \subset \mathbb{P}^{4}$ with non-allowable singularities has codimension $\geqslant 7$ in the space $\mathcal{H y p}_{3,3}$ of all cubic 3 -folds.

(4) $Y$ has finite stabilizer.

Proof. A non-ADE hypersurface singularity has Tjurina number $\tau \geqslant 7$, giving (0).

(1) The simultaneous versality statement (1) is a specialization of a result of ShustinTyomkin [62, main theorem] to the case of cubic 3 -folds (in fact, $\tau_{\text {tot }}(Y) \leqslant 7$ suffices; see also [52, Lemma $3.3(\mathrm{i})]$ which gives the stronger results that $\tau_{\text {tot }}(Y) \leqslant 15$ suffices for cubic 3-folds; [17, p. 35, Fact (5)] gives the simultaneous versality for GIT stable cubics). 
(2) The correspondence of singularities under the projection from a good line is [17, Proposition 3.6]. Clearly, we get $\tau_{\text {tot }}\left(C_{l}\right) \leqslant 6$, which then implies that the deformations of $C_{l}$ give simultaneous versal deformations of its singularities (in fact, [61] says that $\tau_{\text {tot }}\left(C_{l}\right)<4(d-1)=16$ suffices $)$. Finally, the compatibility between the global-to-local deformations of $Y$ and $C_{l}$ is discussed in [16, §3.3].

(3) The expected codimension for the equisingular deformations of a singular cubic $Y$ is $\tau_{\text {tot }}(Y)$. Thus, the locus of cubics with $\tau_{\text {tot }}(Y) \geqslant 7$ is expected to have codimension 7 in $\mathcal{H y p}_{3,3}$. The simultaneous versality statements cited above (more precisely, [52, Lemma 3.3(i)]) guarantee that the expected codimension is the actual codimension for cubics with $\tau_{\text {tot }}(Y) \leqslant 15$. It remains to check that the more degenerate cases (cubics with $\tau_{\text {tot }}(Y)>15$ or non-isolated singularities) have still codimension $\geqslant 7$. This is an easy case-by-case analysis that we omit (the main tool for this analysis is to study a singular cubic via the associated $(2,3)$ complete intersection in $\mathbb{P}^{3}$, see $\left.[17, \S 3.1]\right)$.

(4) The cases when $Y$ is GIT semi-stable (in particular, if $Y$ has at worst $A_{1}, \ldots, A_{5}$ or $D_{4}$ singularities) and has positive-dimensional stabilizer are classified by Allcock (e.g. [3]). It follows that either $\tau_{\text {tot }}(Y) \geqslant 10$ or $Y$ has non-isolated singularities (in fact, $Y$ is the chordal cubic). Assuming that $Y$ is not GIT semi-stable and that $\tau_{\text {tot }}(Y) \leqslant 6$ leads to a small number of cases that can be excluded by a case-by-case analysis. Namely, $Y$ is stabilized either by $G_{m}$ or $G_{a}$. The unipotent case can be seen not to occur using the classification of [53]. Finally, if $Y$ is stabilized by $G_{m}$, then it has at least two singularities. Under our assumptions (in particular, GIT unstable), the only possibility is that $Y$ has a $D_{5}$ singularity and an $A_{1}$ singularity, which can be then excluded by studying the associated $(2,3)$ curve obtained by projecting from the $A_{1}$ singular point.

An immediate consequence of Corollary 2.11, Lemma 3.1 and Proposition $3.6(3)$ is the following.

Corollary 3.7. If $X$ is a general cubic 4-fold, any hyperplane section $Y$ of $X$ is allowable, hence satisfies properties (0)-(4) of Proposition 3.6. Moreover, $Y$ has a very good line.

Note however that since we are restricting to the universal family $\mathcal{Y} / B$ of hyperplane sections of a fixed cubic 4-fold $X$, the simultaneous versal statement of Proposition 3.6 (1) does not suffice for our purposes. What is needed instead is the following lemma which follows from Proposition 3.6 and a transversality argument.

Lemma 3.8. Let $X \subset \mathbb{P}^{5}$ be a general cubic 4-fold, and let $Y$ be any hyperplane section of $X$. Then the natural morphism $H^{0}\left(Y, \mathcal{O}_{Y}(1)\right) \rightarrow \prod_{p \in \operatorname{Sing} Y} T_{Y_{p}}^{1}$ is surjective. In other words, the family of deformations of $Y$ in $X$ induces a versal deformation of the singularities of $Y$. 
Combined with Proposition 3.6 (2), Lemma 3.8 gives the following.

Corollary 3.9. In the situation of Lemma 3.8, denote by $\mathcal{F}_{\text {good }}$ the universal family of good lines in hyperplane sections of $X$ :

$$
\mathcal{F}_{\text {good }}=\left\{([l], t) \in G(2,6) \times B: l \text { is a good line of } Y_{t}\right\}
$$

Then, if $X$ is general, $Y_{0} \subset X$ is any hyperplane section and $l \subset Y_{0}$ is a general good line, the natural map $T_{\mathcal{F}_{\text {good }},([l], 0)} \rightarrow \bigoplus_{p \in \operatorname{Sing} C_{l, Y_{0}}} T_{C_{l, p}}^{1}$ is surjective. Furthermore, for a local analytic or étale section $B \subset \mathcal{F}_{\text {good }}$ of the second projection defined near 0 , the natural map $T_{B,([l], 0)} \rightarrow \bigoplus_{p \in \operatorname{Sing} C_{l, Y_{0}}} T_{C_{l, p}}^{1}$ is surjective.

Proof of Lemma 3.8. Using Lemma 3.1 and Proposition 3.6, we see that there exists a (non-empty) Zariski open subset $\mathcal{H y p}_{4,3}^{00} \subset \mathcal{H y p}{ }_{4,3}^{0}$ of the space of cubic 4 -folds such that: (i) $X$ has trivial automorphism, and (ii) any hyperplane section $Y$ of $X$ has finite stabilizer and satisfies property (1) of Proposition 3.6 (i.e. the space of cubic 3-folds $\mathcal{H} \mathrm{yp}_{3,3}$ gives a simultaneous versal deformation of the singularities of $Y$ ). We want to obtain the stronger statement that the hyperplane sections of $X$ (giving a $\mathbb{P}^{5}$ non-linearly embedded in $\left.\mathcal{H y p}_{3,3}\right)$ give a versal deformation of the singularities of $Y$.

Let $k$ be a number and $z=\left(z_{1}, \ldots, z_{k}\right)$ be the data of $k$ analytic isomorphism classes of germs of allowable hypersurfaces singularities. Let

$$
N:=\sum_{i=1}^{k} \operatorname{dim} T_{z_{i}}^{1}\left(=\tau_{\text {tot }}\right) .
$$

Let $\mathcal{H y p}_{3,3, z} \subset \mathcal{H} \mathrm{yp}_{3,3}$ be the set of cubic 3 -folds admitting exactly $k$ singular points with local germs $z_{i}$. Note that, by Lemma 3.8, we may assume $N \leqslant 6$, i.e. a cubic 3 -fold $Y$ with higher $N$ will not occur as a hyperplane section of a cubic 4 -fold $[X] \in \mathcal{H y p} \mathrm{y}_{4,3}^{0,0}$. Using property (1), we conclude that $\mathcal{H y p}_{3,3, z}$ is smooth locally closed of codimension $N$ in $\mathcal{H} \mathrm{yp}_{3,3}$. It follows that its inverse image $r^{-1}\left(\mathcal{H y p}_{3,3, z}\right)$ is smooth of codimension $N$ in $\mathcal{H} \mathrm{yp}_{4,3}^{00}$. The group PGL $(6,5)$ of automorphisms of $\mathbb{P}^{5}$ preserving $\mathbb{P}^{4}$ acts now on $\mathcal{H}_{\mathrm{yp}}^{00}{ }_{4,3}^{00}$ preserving $r^{-1}\left(\mathcal{H y p}_{3,3, z}\right)$, and using the definition of $\mathcal{H} \mathrm{yp}_{4,3}^{00}$ we find that the fiber $L_{X}$ over a general point $[X] \in \mathcal{H} \mathrm{yp}_{4,3}^{00} / \mathrm{PGL}(6)$ of the quotient map

$$
\mathcal{H} \mathrm{yp}_{4,3}^{00} / \operatorname{PGL}(6,5) \longrightarrow \mathcal{H}_{y p}^{00} / \operatorname{PGL}(6)
$$

is smooth, isomorphic to $\mathbb{P}\left(H^{0}\left(\mathcal{O}_{X}(1)\right)\right.$. (This statement is in fact not completely correct due to the presence of hyperplane sections of $X$ which have finite automorphisms, but it is true at the infinitesimal level.) Sard's theorem then tells us that, for general $X$, the 
locus $L_{X} \cap r^{-1}\left(\mathcal{H y p}_{3,3, z}\right)$ is smooth of codimension $N$ in $L_{X}$, which exactly means that for the given type $z$, and for any $Y \subset X$ having $z$ as singularities, the map

$$
H^{0}\left(Y, \mathcal{O}_{Y}(1)\right) \longrightarrow \prod_{p \in \operatorname{Sing} Y} T_{Y_{p}}^{1}
$$

is surjective. The conclusion then follows from the fact that there are finitely many analytic isomorphism classes of allowable singularities (by Proposition 3.6 (0), all of them are ADE with $\left.\tau_{\text {tot }} \leqslant 6\right)$.

\subsection{Transversality results in the Pfaffian case}

Recall that a Pfaffian cubic hypersurface is a linear section of the Pfaffian cubic hypersurface in $\mathbb{P}^{14}=\mathbb{P}\left(\bigwedge^{2} W_{6}\right)$ defined by the vanishing of $\omega^{3}$ in $\bigwedge^{6} W_{6}$. Pfaffian cubic 4 -folds are parameterized by a hypersurface $\mathcal{P}$ in the moduli space of all smooth cubic 4-folds (see [13]). Restricting to cubic 4-folds without automorphisms, this hypersurface is smooth away from the locus where the cubic has two different Pfaffian structures. In general, it is a divisor with normal crossings, with one branch for each Pfaffian structure. This follows from the fact that the period map for cubic 4 -folds is étale. As we want to apply the results of $\S 4$ also to the case of a general Pfaffian cubic 4 -fold, we have to prove that a general Pfaffian cubic 4-fold satisfies the needed assumptions, namely Lemmas 3.11 and 3.12 , that will be obtained as easy consequences of the following lemma.

Lemma 3.10. Let $X$ be a general Pfaffian cubic 4-fold. Then for any hyperplane section $Y \subset X$ with equation $f_{Y} \in H^{0}\left(X, \mathcal{O}_{X}(1)\right)$, the subspace

$$
f_{Y} H^{0}\left(X, \mathcal{O}_{X}(2)\right) \subset H^{0}\left(X, \mathcal{O}_{X}(3)\right)
$$

is not contained in the tangent space to the Pfaffian hypersurface at the point $[X]$.

The Pfaffian locus $\mathcal{P}$ is an open set in the hypersurface $\mathcal{C}_{14}$ in the space of all cubic 4 -folds parameterizing special cubics with discriminant 14 (see [32]). The subspace

$$
f_{Y} H^{0}\left(X, \mathcal{O}_{X}(2)\right) \subset H^{0}\left(X, \mathcal{O}_{X}(3)\right)
$$

is the space of first-order deformations of $X$ containing $Y$.

Proof of Lemma 3.10. The Pfaffian cubic 4-folds are characterized by the fact that they contain quintic del Pezzo surfaces: if $X$ contains a quintic del Pezzo surface $\Sigma$, the Pfaffian rank 2 vector bundle $\mathcal{E}$ with $c_{2}=2$ and $c_{1}=0$ on $X$ is deduced from $\Sigma$ by the Serre construction. Conversely, if $X$ is Pfaffian with Pfaffian rank-2 vector bundle $\mathcal{E}$, 
there is a 5 -dimensional family of quintic del Pezzo surfaces $\Sigma$ in $X$, obtained as zero-sets of sections of $\mathcal{E}$ (see [11, Proposition 9.2]). Let $\Sigma \subset X$ be such a pair, and let $\sigma:=[\Sigma] \in$ $H^{2}\left(X, \Omega_{X}^{2}\right)$ be the cohomology class of $\Sigma$. The numerical condition characterizing the Pfaffian class $\sigma$ is $\sigma^{2}=13$ and $\sigma \cdot h^{2}=5$, where we use the intersection pairing on $H^{4}(X, \mathbb{Z})$ and $h=c_{1}\left(\mathcal{O}_{X}(1)\right)$. The cup-product with $\sigma$ induces a composite morphism

$$
\sigma \cup(\cdot): H^{0}\left(X, \mathcal{O}_{X}(3)\right) \stackrel{\varrho}{\longrightarrow} H^{1}\left(X, T_{X}\right) \longrightarrow H^{1,3}(X),
$$

where the first map $\varrho$ is the Kodaira-Spencer map, and the general theory of variations of Hodge structures tells us that the tangent space to the Pfaffian locus $\mathcal{P}$ at $[X]$ identifies to $\operatorname{Ker} \sigma \cup(\cdot)$. Note that $\varrho$ identifies to the quotient map

$$
H^{0}\left(X, \mathcal{O}_{X}(3)\right) \longrightarrow R_{f}^{3}:=H^{0}\left(X, \mathcal{O}_{X}(3)\right) / J_{f}^{3}
$$

where $f=0$ is the defining equation for $X$ and $J_{f}^{3}$ is the degree-3 piece of the Jacobian ideal of $f$. Griffiths' residue theory (see [65, Vol. II, §6.2]) provides isomorphisms

$$
H^{2,2}(X)_{\text {prim }} \cong R_{f}^{3} \quad \text { and } \quad H^{1,3}(X) \cong R_{f}^{6}
$$

such that the second map in (3.1) identifies to multiplication by $\tilde{\sigma}: R_{f}^{3} \rightarrow R_{f}^{6}$, where $\tilde{\sigma} \in R_{f}^{3}$ is the representative of $\sigma$, or rather of its projection in $H^{2,2}(X)_{\text {prim }}=H^{2,2}(X) /\left\langle h^{2}\right\rangle$. Lemma 3.10 can thus be rephrased as follows: For a general Pfaffian cubic 4-fold with equation $f=0$, the Pfaffian class $\tilde{\sigma} \in R_{f}^{3}$ is not annihilated by $y R_{f}^{2}$, for any non-zero $y \in H^{0}\left(X, \mathcal{O}_{X}(1)\right)$. Note that by Macaulay's theorem [65, Vol. II, $\left.\S 6.2 .2\right]$, to say that $y \tilde{\sigma} R_{f}^{2}=0$ in $R_{f}^{6}$ is equivalent to saying that $y \tilde{\sigma}=0$ in $R_{f}^{4}$. So what we have to prove is the following claim.

Claim. For a general Pfaffian cubic 4-fold with equation $f$ and (primitive) Pfaffian class $\tilde{\sigma} \in R_{f}^{3}$, and for any non-zero $y \in H^{0}\left(X, \mathcal{O}_{X}(1)\right)$, one has y $\tilde{\sigma} \neq 0$ in $R_{f}^{4}$.

In order to prove the claim, we use the fact $\left({ }^{1}\right.$ ) (see [32]) that cubic 4-folds containing two non-intersecting planes $P_{1}$ and $P_{2}$ are parameterized by points in the closure of the divisor $\mathcal{C}_{14}$. In fact, if $p_{i}$ is the cohomology class of $P_{i}, i=1,2$, one has

$$
p_{i}^{2}=3, \quad p_{1} \cdot p_{2}=0, \quad h^{2} \cdot p_{i}=1
$$

and so $\sigma=h^{2}+p_{1}+p_{2}$ satisfies the numerical conditions $\sigma^{2}=13$ and $\sigma \cdot h^{2}=5$. It thus suffices to prove that, for a general cubic 4-fold containing two non-intersecting planes $P_{1}$ and $P_{2}$, and for any non-zero $y \in S^{1}:=H^{0}\left(X, \mathcal{O}_{X}(1)\right)$, the class $h+p_{1}+p_{2} \in H^{2,2}(X)_{\text {prim }}=R_{f}^{3}$

$\left({ }^{1}\right)$ We are grateful to the referee for pointing out this fact, which simplified our original argument. 
satisfies $y\left(h+p_{1}+p_{2}\right) \neq 0$ in $R_{f}^{4}$. This computation can be made explicitly on the Fermat cubic $X_{f}$ with equation $f=\sum_{i=0}^{5} x_{i}^{3}=0$, where such configurations of planes are easy to exhibit: we can take $P_{1}$ to be defined by $x_{0}=\zeta x_{1}, x_{2}=\zeta x_{3}, x_{4}=\zeta x_{5}$, with $\zeta^{3}=-1$, and $P_{2}$ to be defined by $x_{0}=\zeta^{\prime} x_{1}, x_{2}=\zeta^{\prime} x_{3}, x_{4}=\zeta^{\prime} x_{5}$, with $\left(\zeta^{\prime}\right)^{3}=-1$, and $\zeta^{\prime} \neq \zeta$. The computations in the Jacobian ring $R_{f}$ are easy to perform. In this ring, $x_{i}^{2}=0$, hence in every degree $\leqslant 6$, we get as free generators the monomials $\prod_{i \in I} x_{i}$ with no repeated indices. The primitive class $\tilde{p}_{1} \in R_{f}^{3}$ defined as the projection of $p_{1}$ is annihilated by multiplication by $x_{0}-\zeta x_{1}, x_{2}-\zeta x_{3}, x_{4}-\zeta x_{5}$, because these are hyperplane sections vanishing on $P_{1}$, and similarly the primitive class $\tilde{p}_{2}$ of $p_{2}$ is annihilated by multiplication by $x_{0}-\zeta^{\prime} x_{1}$, $x_{2}-\zeta^{\prime} x_{3}, x_{4}-\zeta^{\prime} x_{5}$. It follows that $S^{1} \cdot \tilde{p}_{1} \subset R_{f}^{4}$ is orthogonal with respect to Macaulay duality (see [65, Vol. II, §6.2.2]) to the subspace

$$
S^{1} \cdot\left\langle x_{0}-\zeta x_{1}, x_{2}-\zeta x_{3}, x_{4}-\zeta x_{5}\right\rangle \subset R_{f}^{2}
$$

and similarly for $p_{2}$. But then the two spaces $S^{1} \cdot \tilde{p}_{1}$ and $S^{1} \cdot \tilde{p}_{2}$ have trivial intersection, as otherwise the spaces

$$
S^{1} \cdot\left\langle x_{0}-\zeta x_{1}, x_{2}-\zeta x_{3}, x_{4}-\zeta x_{5}\right\rangle \quad \text { and } \quad S^{1} \cdot\left\langle x_{0}-\zeta^{\prime} x_{1}, x_{2}-\zeta^{\prime} x_{3}, x_{4}-\zeta^{\prime} x_{5}\right\rangle
$$

would not generate $R_{f}^{2}$. Thus, if $y \in S^{1}$ satisfies $y\left(\tilde{p}_{1}+\tilde{p}_{2}\right)=0$ in $R_{f}^{4}$, one has

$$
y \tilde{p}_{1}=0 \text { and } y \tilde{p}_{2}=0 \text { in } R_{f}^{4} \text {. }
$$

This easily implies that $y=0$.

We have the following applications.

Lemma 3.11. Let $X$ be a general Pfaffian cubic 4-fold. Then for any hyperplane section $Y$ of $X$, the natural map $H^{0}\left(Y, \mathcal{O}_{Y}(1)\right) \rightarrow \bigoplus_{p \in \operatorname{Sing} Y} T_{Y_{p}}^{1}$ is surjective.

Proof. Let $\mathcal{P}^{00}$ be the Zariski open subset of the Pfaffian locus, which is defined as the intersection of $\mathcal{P}$ with the Zariski open set $\mathcal{H y p}{ }_{4,3}^{00}$. Note that $\mathcal{P}^{00}$ is non-empty by Lemma 3.1 (ii), using the fact that the set of $[Y] \in \mathbb{P}\left(H^{0}\left(\mathcal{O}_{\mathbb{P}^{4}}(3)\right)\right)$ admitting a nontrivial vector field has codimension $\geqslant 7$ (see Proposition 3.6 (4)). We now consider the natural map $r_{\mathrm{pf}}: \mathcal{P}^{00} \rightarrow \mathcal{H y p}_{3,3}$, defined as the restriction to $\mathcal{P}^{00} \subset \mathcal{H y p} \mathrm{p}_{4,3}^{00}$ of $r: \mathcal{H y p}_{4,3}^{00} \rightarrow$ $\mathcal{H} \mathrm{yp}_{3,3}$. The fiber of $r_{\mathrm{pf}}$ over $[Y] \in \mathcal{H} \mathrm{yp}_{3,3}$ consists in those Pfaffian cubic 4-folds which intersect $\mathbb{P}^{4}$ along $Y$. Let $X$ be a general Pfaffian cubic 4-fold. Then $r_{\mathrm{pf}}$ is smooth at any $\left[X^{\prime}\right]$ parameterizing a cubic isomorphic to $X$. Indeed, the map $r$ is smooth, and $r_{\mathrm{pf}}$ is the restriction of $r$ to $\mathcal{P}^{00}$. Thus, if $r_{\mathrm{pf}}$ was not smooth at a point $\left[X^{\prime}\right]$ with $[Y]=r_{\mathrm{pf}}\left(\left[X^{\prime}\right]\right)$, then the fiber of $r$ would be tangent to $\mathcal{P}^{00}$ at $\left[X^{\prime}\right]$, which exactly means that $f_{Y} H^{0}\left(X^{\prime}, \mathcal{O}_{X^{\prime}}(2)\right) \subset H^{0}\left(X^{\prime}, \mathcal{O}_{X^{\prime}}(3)\right)$ is contained in the tangent space to the 
Pfaffian hypersurface at the point $\left[X^{\prime}\right]$. As $X$ is general, Lemma 3.11 tells us that this does not happen at any $X^{\prime}$ isomorphic to $X$. The end of the proof is now identical to the proof of Lemma 3.8. Indeed, by Lemma 3.12 below and Proposition 3.6, property (1) of Proposition 3.6 is satisfied by any hyperplane section of a general Pfaffian cubic, replacing $r^{-1}\left(\mathcal{H} \mathrm{yp}_{3,3, z}\right)$ by its Pfaffian analogue $r_{\mathrm{pf}}^{-1}\left(\mathcal{H} \mathrm{yp}_{3,3, z}\right)$, which we know to be smooth.

Recall from Definition 2.9 that a very good line in a cubic 3 -fold containing no plane is a line which is good and such that the curve $\widetilde{C}_{l}$ of lines in $Y$ meeting $l$ is irreducible.

Lemma 3.12. Let $X$ be a general Pfaffian cubic 4-fold. Then any hyperplane section $Y$ of $X$ is allowable and admits a very good line.

Proof. We know by Propositions 2.3 and 2.10 and Corollary 3.7 that if $X$ is a general cubic 4-fold, then any hyperplane section of $X$ is allowable and contains a very good line. Let us say that $Y$ is bad if it does not admit a very good line or has non-allowable singularities and $X$ is bad if it has a hyperplane section $Y$ which is bad. The locus of bad cubic 4 -folds is a proper closed algebraic subset $\mathcal{H y p}_{4,3}^{00, \text { bad }}$ of $\mathcal{H y p}{ }_{4,3}^{00}$, and its irreducible components are constructed as follows: for each irreducible component $Z \subset \mathcal{H y p}_{3,3}$ of the locus of bad cubic 3 -folds, $r^{-1}(Z) \subset \mathcal{H y p}{ }_{4,3}$ is the locus of bad cubic 4 -folds such that the cubic 3 -fold $X \cap \mathbb{P}^{4}$ is parameterized by a point of $Z$. Thus $\operatorname{PGL}(6) \cdot r^{-1}(Z)$ is the set of cubic 4 -folds $X$ such that some hyperplane section of $X$ is isomorphic to a cubic 3-fold parameterized by a point of $Z$. It is thus clear that we get any irreducible component of $\mathcal{H y p}_{4,3}^{00, \text { bad }}$ as $\operatorname{PGL}(6) \cdot r^{-1}(Z)$, with $Z$ as above. We conclude from this that any irreducible component $Z^{\prime}$ of $\mathcal{H y p} p_{4,3}^{00, \text { bad }}$ has the property that, for any $[X] \in Z^{\prime}$, there exists a hyperplane section $Y \subset X$ such that all cubic 4-folds containing $Y$ as a hyperplane section are parameterized by points of $Z^{\prime}$. In particular, if $f_{Y} \in H^{0}\left(X, \mathcal{O}_{X}(1)\right)$ is the equation of $Y$ in $X, f_{Y} H^{0}\left(X, \mathcal{O}_{X}(2)\right)$ must be contained in the Zariski tangent space of $Z^{\prime}$. If all Pfaffian cubic 4 -folds were bad, then the Pfaffian hypersurface $\mathcal{P}^{00}$ would be an irreducible component $Z^{\prime}$ of $\mathcal{H y p}_{4,3}^{00, \text { bad }}$, and we would get a contradiction with Lemma 3.10 .

\section{Relative compactified Prym varieties}

As previously mentioned, our main tool for compactifying the intermediate Jacobian fibration $\mathcal{J}_{U} \rightarrow U$ is the Prym construction that identifies the intermediate Jacobian $J(Y)$ with a Prym variety $\operatorname{Prym}(\widetilde{C} / C)$ (where the pair $(\widetilde{C}, C)$ is obtained from $Y$ via the projection from a general line). The Prym construction works well in a relative setting over the smooth locus $U \subset\left(\mathbb{P}^{5}\right)^{\vee}$ (and more precisely over the open set $\mathcal{F}^{0} / U$ of very good lines in the fibers), reducing (at least locally) the problem of understanding degenerations 
of intermediate Jacobians to that of understanding degenerations of Prym varieties. This is of course a well studied problem: Beauville [8] gave a compactification of the moduli of pairs $(\widetilde{C}, C)$, and many people studied degenerations of Pryms as abelian varieties (e.g. [24], [2], [15]). Here we need to understand a specific compactification, and its local structure, over a given base $B$. A few instances of this have already been studied in [45], [7], and [58]. Below, we define and prove a number of results for the relative compactified Prym of families of étale double covers of irreducible curves, which we then apply to our context. Specifically, the results of $\S 2$ and $\S 3$ say that, for a general cubic 4 -fold $X$, we may replace (locally on $B$ ) the family $\mathcal{Y} / B$ of hyperplane sections by a family of double covers $(\widetilde{\mathcal{C}}, \mathcal{C})$ such that each fiber $\left(\widetilde{C}_{t}, C_{t}\right)$ is an étale double cover with both curves irreducible (see Corollary 2.11). Furthermore, the singularities of $\widetilde{C}_{t}$ and $C_{t}$ are planar, and we may assume (see Corollary 3.9 ) that the family $\mathcal{C}$ gives a simultaneous versal deformation of the singularities of any fiber $C_{t}$.

Notation 4.1. From now on in this section, $B$ will stand for an arbitrary base, not necessarily $\left(\mathbb{P}^{5}\right)^{\vee}$ as elsewhere in the paper.

We proceed as follows: as in [7] we can define, for any family $\widetilde{\mathcal{C}}_{B} \rightarrow \mathcal{C}_{B}$ of étale double covers of irreducible locally planar curves, parameterized by a base $B$, a relative compactified Prym variety $\operatorname{Prym}\left(\widetilde{\mathcal{C}}_{B} / \mathcal{C}_{B}\right) \rightarrow B$ whose fibers over the locus parameterizing smooth curves are usual Prym varieties. The relative Prym variety is defined as (one component) of the fixed locus of an involution on the relative compactified Jacobian $\overline{\operatorname{Jac}}\left(\widetilde{\mathcal{C}}_{B}\right)$ of the family $\widetilde{\mathcal{C}}_{B} \rightarrow B$. From this definition, it follows immediately that, if $\overline{\operatorname{Jac}}\left(\widetilde{\mathcal{C}}_{B}\right)$ is smooth, then so is $\operatorname{Prym}\left(\widetilde{\mathcal{C}}_{B} / \mathcal{C}_{B}\right)$. Unfortunately, in general $\overline{\operatorname{Jac}}\left(\widetilde{\mathcal{C}}_{B}\right)$ is not smooth. However, one can sometimes think of $\widetilde{\mathcal{C}}_{B} \rightarrow B$ as the restriction of a larger family $\widetilde{\mathcal{C}}_{\widetilde{B}} \rightarrow \widetilde{B}$, $B \subset \widetilde{B}$, with the property that

(a) there exist two compatible involutions on $\widetilde{\mathcal{C}}_{\widetilde{B}}$ and on $\widetilde{B}$, such that the first one is an extension of the given involution on $\widetilde{\mathcal{C}}_{B}$ and second one has the property that the fixed locus on $\widetilde{B}$ is equal to $B$;

(b) the relative compactified Jacobian $\overline{\operatorname{Jac}}\left(\widetilde{\mathcal{C}}_{\widetilde{B}}\right)$ is smooth. Under these assumptions the relative Prym variety $\operatorname{Prym}\left(\widetilde{\mathcal{C}}_{B} / \mathcal{C}_{B}\right)$ is smooth. An instance of this already appeared in [7]. As discussed below, the versality statements valid in our setup allow us to conclude that the relative compactified Prym variety is indeed smooth in our situation.

The results in this section build on an important result for compactified relative Jacobians, namely the Fantechi-Göttsche-van Straten [23] smoothness criterion. 


\subsection{Relative compactified Prym varieties (the étale case)}

Let $f: \widetilde{C} \rightarrow C$ be an étale double cover of smooth projective curves, and let $\iota: \widetilde{C} \rightarrow \widetilde{C}$ be the corresponding involution on $\widetilde{C}$. We denote by $g$ be the genus of $C$, and by $h$ the genus of $\widetilde{C}$, so that $h=2 g-1$. Recall that the Prym variety of $\widetilde{C}$ over $C$, which we will denote by $\operatorname{Prym}(\widetilde{C} / C)$, is the identity component of the fixed locus of the involution

$$
\tau:=-\iota^{*}: \operatorname{Pic}^{0}(\widetilde{C}) \longrightarrow \operatorname{Pic}^{0}(\widetilde{C}) .
$$

The Prym variety $\operatorname{Prym}(\widetilde{C} / C)$ is a principally polarized abelian variety [49] of dimension $g-1$. Equivalently [49], the Prym variety can be defined as the identity component of the norm map

$$
\begin{aligned}
& \mathrm{Nm}: \operatorname{Pic}^{0}(\mathcal{C}) \longrightarrow \operatorname{Pic}^{0}(C), \\
& \mathcal{O}_{\widetilde{C}}\left(\sum_{i} p_{i}\right) \longmapsto \mathcal{O}_{C}\left(\sum f\left(p_{i}\right)\right),
\end{aligned}
$$

or as the image of

$$
1-\iota^{*}: \operatorname{Pic}^{0}(\widetilde{C}) \longrightarrow \operatorname{Pic}^{0}(\widetilde{C}) .
$$

Now suppose that $f: \widetilde{C} \rightarrow C$ is an étale double cover of singular, but irreducible curves, and let $\tilde{n}: \widetilde{D} \rightarrow \widetilde{C}$ and $n: D \rightarrow C$ be the normalizations of the two curves. The involution $\iota$ on $\widetilde{C}$ lifts to a compatible involution

$$
\varepsilon: \widetilde{D} \longrightarrow \widetilde{D}
$$

so that the natural morphism $\widetilde{D} \rightarrow D$ is an étale double cover with associated involution $\varepsilon$. Let $\left\{x_{1}, \ldots, x_{k}\right\}$ be the singular points of $C$ and let

$$
\left\{p_{1}, \ldots, p_{k}, q_{1}, \ldots, q_{k}\right\}
$$

be the singular points of $\widetilde{C}$, with $f^{-1}\left(x_{i}\right)=\left\{p_{i}, q_{i}\right\}$. The identity component of the Picard group, or generalized Jacobian, of $\widetilde{C}$ fits into the natural short exact sequence of groups

$$
1 \longrightarrow A \times A \longrightarrow \operatorname{Pic}^{0}(\widetilde{C}) \stackrel{\tilde{n}^{*}}{\longrightarrow} \operatorname{Pic}^{0}(\widetilde{D}) \longrightarrow 1,
$$

where

$$
A:=H^{0}\left(\widetilde{C}, \bigoplus_{i=1}^{k}\left(n_{*} \mathcal{O}_{\widetilde{D}}^{\times} / \mathcal{O}_{\widetilde{C}}^{\times}\right)_{p_{i}}\right) \cong H^{0}\left(\widetilde{C}, \bigoplus_{i=1}^{k}\left(n_{*} \mathcal{O}_{\widetilde{D}}^{\times} / \mathcal{O}_{\widetilde{C}}^{\times}\right)_{q_{i}}\right)
$$

is a commutative affine group. The involution $-\iota^{*}$ still acts on $\operatorname{Pic}^{0}(\widetilde{C})$, and we can define, in analogy with the generalized Jacobian, the generalized Prym variety of $\widetilde{C}$ over $C$ to be the identity component of the fixed locus of $-\iota^{*}$ :

$$
\operatorname{Prym}(\widetilde{C} / C):=\operatorname{Fix}\left(-\iota^{*}\right)_{0} .
$$


The involution $-\iota^{*}$ is compatible, via $n^{*}$, with $-\varepsilon^{*}$ on $\operatorname{Pic}^{0}(\widetilde{D})$ and it acts on $A \times A$ via $(a, b) \mapsto\left(b^{-1}, a^{-1}\right)$ (note that we use multiplicative notation for these groups, even though $A$ can be a product of both additive and multiplicative groups). We therefore get a short exact sequence

$$
1 \longrightarrow A \longrightarrow \operatorname{Prym}(\widetilde{C} / C) \stackrel{\tilde{n}^{*}}{\longrightarrow} \operatorname{Prym}(\widetilde{D} / D) \longrightarrow 1,
$$

where the inclusion $A \hookrightarrow A \times A=\operatorname{ker} \tilde{n}^{*}$ is given by $a \mapsto\left(a, a^{-1}\right)$. Set $\delta:=\operatorname{dim} A$, so that $g(D)=g-\delta$ and $g(\widetilde{D})=h-2 \delta$. Since $\operatorname{dim} \operatorname{Prym}(\widetilde{D} / D)=g-\delta-1$, we see that

$$
\operatorname{dim} \operatorname{Prym}(\widetilde{C} / C)=g-1,
$$

as in the smooth case. For example, if $C$ is a nodal irreducible curve, then $\operatorname{Prym}(\widetilde{C} / C)$ is a semi-abelian variety.

The strategy to compactify the generalized Prym variety is to extend the involution to the compactified Jacobian of $\widetilde{C}$. By definition, the degree- $d$ compactified Jacobian of an integral projective curve $\Gamma$ is the moduli space of rank- 1 , degree- $d$, torsion-free sheaves on $\Gamma$. If $\Gamma$ has planar singularities, i.e. if locally around every singular point, $\Gamma$ is isomorphic to a plane curve, then every component is irreducible [56] of dimension equal to the arithmetic genus of $\Gamma$. The degree-zero component $\overline{\operatorname{Jac}}(\Gamma)$ contains $\operatorname{Pic}^{0}(\Gamma)$ as an open dense subset. For the proof of Propositions 4.10 and 4.20 we will need further properties of the compactified Jacobian, and in particular we will need a description of the complement $\overline{\operatorname{Jac}}(\Gamma) \backslash \operatorname{Pic}^{0}(\Gamma)$ (cf. [56] and [20]; see Proposition 4.11 below), and a smoothness criterion due to Fantechi-Göttsche-van Straten [23] (recalled below in Proposition 4.19). If $\Gamma$ has locally planar singularities, then any torsion-free coherent sheaf $F$ on $\Gamma$ is reflexive, that is

$$
\left(F^{\vee}\right)^{\vee}=F, \quad \text { where } F^{\vee}:=\mathcal{H}_{\operatorname{om}_{\mathcal{O}_{\Gamma}}}\left(F, \mathcal{O}_{\Gamma}\right)
$$

Moreover, if

$$
j: \Gamma \subset Z,
$$

is an embedding of $\Gamma$ in a smooth projective variety $Z$ of dimension $d$, then using [34, Proposition 1.1.10] we can see that

$$
\mathcal{E x t}_{Z}^{c}\left(F, \omega_{Z}\right)=0 \text { for all } c \neq d-1 \text {. }
$$

The following lemma is well known, and is crucial to define the involution as a regular morphism on the family of compactified Jacobians. 
LEMma 4.2. The only non-zero $\mathcal{E}$ xt sheaf satisfies

$$
\mathcal{E}_{\mathrm{xt}_{Z}^{d-1}}\left(F, \mathcal{O}_{Z}\right)=F^{\vee} \otimes \operatorname{det} N_{\Gamma \mid Z}
$$

(notice that, since $\Gamma$ has locally planar singularities, the embedding in $Z$ is a local complete intersection (l.c.i.) variety).

Proof. This is simply [31, Chapter III, Lemma 7.4 and Theorem 7.11]. The only thing to remark is that the proof of [31, Lemma 7.4] goes through unchanged for the ext-sheaves.

Lemma 4.3. Assume that $C$, and therefore $\widetilde{C}$, has planar singularities. Then the assignment

$$
\begin{gathered}
\tau: \overline{\operatorname{Jac}}(\widetilde{C}) \longrightarrow \overline{\operatorname{Jac}}(\widetilde{C}), \\
F \longmapsto \iota^{*} F^{\vee}
\end{gathered}
$$

defines a regular involution which extends $-\iota^{*}$ over the complement of $\operatorname{Pic}^{0}(\widetilde{C})$.

Proof. By [4, Theorem 3.4], there is a universal sheaf $\mathcal{F}$ on $\overline{\operatorname{Jac}}(\widetilde{C}) \times \widetilde{C}$. Let $\widetilde{C} \subset Z$ be an embedding of $\widetilde{C}$ in a smooth projective variety $Z$ of dimension $d \geqslant 2$. We may view $\mathcal{F}$ as a sheaf on $\overline{\operatorname{Jac}}(\widetilde{C}) \times Z$, i.e., as a flat family of pure codimension- $(d-1)$ sheaves on $Z$ parameterized by $\overline{\operatorname{Jac}}(\widetilde{C})$. Let $p_{Z}: \operatorname{Jac}(\widetilde{C}) \times Z \rightarrow Z$ be the second projection. We claim that the sheaf $\mathcal{E}_{\mathrm{xt}} \frac{d-1}{\operatorname{Jac}(\widetilde{C}) \times Z}\left(\mathcal{F}, p_{Z}^{*} \mathcal{O}_{Z}\right)$ satisfies base change, i.e. that

$$
\mathcal{E x t}_{\overline{\operatorname{Jac}}(\widetilde{C}) \times Z}^{d-1}\left(\mathcal{F}, p_{Z}^{*} \mathcal{O}_{Z}\right)_{\mid\{t\} \times Z}=\mathcal{E x t}^{d-1}\left(\mathcal{F}_{t}, \mathcal{O}_{Z}\right)=\left.\mathcal{F}_{t}^{\vee} \otimes \operatorname{det} N_{C}\right|_{Z}
$$

Indeed, by [5, Theorem 1.10], it is enough to verify that $\mathcal{E x t}^{c}\left(\mathcal{F}_{t}, \mathcal{O}_{Z}\right)=0$, for $c=d-2$ and $c=d$, which follows directly from (4.3).

Using Lemma 4.2 we see that the sheaf

$$
\left.\mathcal{E}_{\mathrm{xt}} \frac{d-1}{\operatorname{Jac}(\widetilde{C}) \times Z}\left(\iota^{*} \mathcal{F}, p_{Z}^{*} \mathcal{O}_{Z}\right) \otimes p_{Z}^{*} \operatorname{det} N_{C}^{\vee}\right|_{Z}
$$

provides a flat family of rank-1 torsion-free sheaves on $C$, and it determines a morphism $\overline{\operatorname{Jac}}(\widetilde{C}) \rightarrow \overline{\operatorname{Jac}}(\widetilde{C})$ which sends a sheaf $F$ to $\iota^{*} F^{\vee}$. Since the sheaves are reflexive, this morphism is an involution.

Definition 4.4. The compactified Prym variety $\overline{\operatorname{Prym}}(\widetilde{C} / C)$ of an étale double cover $\widetilde{C} \rightarrow C$ of integral curves with planar singularities is the irreducible component containing the identity of the fixed locus $\operatorname{Fix}(\tau) \subset \overline{\operatorname{Jac}}(\widetilde{C})$. 
Remark 4.5. Notice that $\operatorname{Prym}(\widetilde{C} / C) \subset \overline{\operatorname{Prym}}(\widetilde{C} / C)$ is a dense open subset, so that, in particular,

$$
\operatorname{dim} \overline{\operatorname{Prym}}(\widetilde{C} / C)=g-1 .
$$

For example, if $C$ is irreducible with one node, then $\overline{\operatorname{Prym}}(\widetilde{C} / C)$ is a rank-1 degeneration of an abelian variety. For an explicit description of Prym varieties of other singular curves, see [58].

Remark 4.6. One could also drop the assumption of integrality, and define the relative compactified Prym variety for étale double covers of arbitrary curves with locally planar singularities. In this situation, however, a choice has to be made, namely that of a polarization on the family of double covers and the relative compactified Prym depends on this choice (for the case of family of curves lying on smooth projective surfaces, see [7] and [58]). Since the curves we will deal with in our situation turn out to be integral by Proposition 2.10, we will restrict ourselves to the case of integral curves.

Now let $B$ be an irreducible base scheme and let

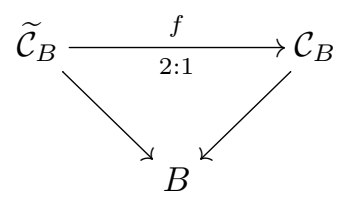

be a family, parameterized by $B$, of étale double covers of reduced and irreducible curves with planar singularities. Let us denote by $g$ the genus of the curves in the family $\mathcal{C}_{B} \rightarrow B$, and by $h=2 g-1$ the genus of their double covers $\widetilde{\mathcal{C}}_{B} \rightarrow B$. We let

$$
\iota: \widetilde{\mathcal{C}}_{B} \longrightarrow \widetilde{\mathcal{C}}_{B}
$$

be the involution associated with the covering, and we define a 2-torsion line bundle $\eta_{B}$ on $\mathcal{C}_{B}$ by setting

$$
f_{*} \mathcal{O}_{\widetilde{\mathcal{C}}_{B}}=\mathcal{O}_{\mathcal{C}_{B}} \oplus \eta_{B}
$$

For any $b \in B$, we denote by

$$
f_{b}: \widetilde{\mathcal{C}_{b}} \longrightarrow \mathcal{C}_{b}, \quad \iota_{b}: \widetilde{\mathcal{C}_{b}} \longrightarrow \widetilde{\mathcal{C}_{b}} \quad \text { and } \quad \eta_{b} \in \operatorname{Pic}^{0}\left(\mathcal{C}_{b}\right)
$$

the restrictions of $f, \iota$ and $\eta$, respectively, to the fiber over $b$. Consider the degree-zero relative compactified Jacobians

$$
\xi: \overline{\mathrm{Jac}}\left(\widetilde{\mathcal{C}}_{B}\right) \longrightarrow B \quad \text { and } \quad \pi: \overline{\mathrm{Jac}}\left(\mathcal{C}_{B}\right) \longrightarrow B
$$


The fiber of $\xi$ (resp. $\pi$ ) over a point $b \in B$ is the compactified Jacobian of the curve $\widetilde{\mathcal{C}}_{b}$ (resp. $\mathcal{C}_{b}$ ) and is a reduced and irreducible l.c.i. variety of dimension $h$ (respectively $g$ ). The smooth locus of $\xi$ (respectively $\pi$ ) is the relative generalized Jacobian $\operatorname{Pic}_{\widetilde{\mathcal{C}}_{B}}^{0}\left(\right.$ resp. $\operatorname{Pic}_{\mathcal{C}_{B}}^{0}$ ) which parameterizes line bundles. Since these fibrations are group schemes, both admit a zero section, and the morphisms $-\iota^{*}$ and $1-\iota^{*}$ are well defined group homomorphisms of $\mathrm{Pic}_{\widetilde{\mathcal{C}}_{B}}^{0}$. Also, $\mathrm{Pic}_{\mathcal{C}_{B}}^{0}$ has another section determined by $\eta_{B}$.

LEMMA 4.7. The "-1" morphism on the group scheme $\mathrm{Pic}_{\widetilde{\mathcal{C}}_{B}}^{0}$ extends to a regular morphism on the whole compactified Jacobian $\overline{\operatorname{Jac}}\left(\widetilde{\mathcal{C}}_{B}\right)$. In particular, there is a regular involution

$$
\begin{aligned}
\tau: \overline{\operatorname{Jac}}\left(\widetilde{\mathcal{C}}_{B}\right) & \longrightarrow \overline{\operatorname{Jac}}\left(\widetilde{\mathcal{C}}_{B}\right) \\
F & \longmapsto \iota^{*} F^{\vee}
\end{aligned}
$$

Proof. Again, this follows from [4, Theorem 3.4]. Locally on $B$ we can find a section of the smooth locus of $\widetilde{\mathcal{C}}_{B} \rightarrow B$, hence by [4] there is a universal sheaf on $\overline{\operatorname{Jac}}\left(\widetilde{\mathcal{C}}_{B}\right) \times \widetilde{\mathcal{C}}_{B}$. Since the morphism $\widetilde{\mathcal{C}}_{B} \rightarrow B$ is projective, we can find (up to restricting $B$ ) a smooth projective variety $Z$ of dimension $d$ such that $\widetilde{\mathcal{C}}_{B} \rightarrow B$ can be factored by an embedding $\widetilde{\mathcal{C}}_{B} \subset Z \times B$ followed by the second projection to $B$. We can hence argue as in Lemma 4.3 and define an involution by considering the flat family of torsion-free sheaves

$$
\mathcal{E}_{\mathrm{xt}}^{d-1}\left(\iota^{*} \mathcal{F}, p_{Z}^{*} \mathcal{O}_{Z}\right) \otimes \operatorname{det} N_{\widetilde{\mathcal{C}}_{B}}^{\vee} \mid Z \times B
$$

Since the assignment is independent of the choice of a section and of $Z$, these local morphisms glue together to a global involution on $\overline{\operatorname{Jac}}\left(\widetilde{\mathcal{C}}_{B}\right)$.

We can now define the relative compactified Prym variety.

Definition 4.8. Let $f: \widetilde{\mathcal{C}}_{B} \rightarrow \mathcal{C}_{B}$ be as in (4.5) an étale double cover between two families of reduced and irreducible curves with planar singularities. The relative compactified Prym variety of $\widetilde{\mathcal{C}}_{B}$ over $\mathcal{C}_{B}$, denoted $\overline{\operatorname{Prym}}\left(\widetilde{\mathcal{C}}_{B} / \mathcal{C}_{B}\right)$, is defined to be the irreducible component of the fixed locus $\operatorname{Fix}(\tau) \subset \overline{\operatorname{Jac}}\left(\widetilde{C}_{B}\right)$ that contains the zero section:

$$
\overline{\operatorname{Prym}}\left(\widetilde{\mathcal{C}}_{B} / \mathcal{C}_{B}\right):=\operatorname{Fix}(\tau)_{0} \subset \overline{\operatorname{Jac}}\left(\widetilde{C}_{B}\right) .
$$

LEMma 4.9. The fixed locus $\operatorname{Fix}\left(-\iota^{*}\right) \subset \operatorname{Pic}_{\widetilde{\mathcal{C}}_{B}}^{0}$ has four isomorphic connected components.

Proof. In [49], it is proved that, for any $b \in B$ such that $\mathcal{C}_{b}$ is smooth,

$$
\mathrm{Nm}^{-1}\left(\mathcal{O}_{\mathcal{C}_{b}}\right) \subset \operatorname{Pic}_{\widetilde{\mathcal{C}}_{b}}^{0}
$$


has two connected components. Hence, so has $\mathrm{Nm}^{-1}\left(\eta_{b}\right)$. Moreover,

$$
f^{*}\left(\operatorname{Nm}\left(\mathcal{O}_{\widetilde{C}_{b}}\left(\sum_{i} c_{i}\right)\right)\right)=\mathcal{O}_{\widetilde{C}_{b}}\left(\sum_{i}\left(c_{i}+\iota c_{i}\right)\right)
$$

and $\operatorname{ker}\left(f^{*}\right)=\left\langle\mathcal{O}_{\widetilde{C}_{b}}, \eta_{b}\right\rangle$, so that $\operatorname{Fix}\left(-\iota_{b}^{*}\right)=\mathrm{Nm}^{-1}\left(\eta_{b}\right) \cup \mathrm{Nm}^{-1}\left(\mathcal{O}_{\mathcal{C}_{b}}\right)$, and hence the fixed locus of $-\iota_{b}^{*}$ in $\operatorname{Pic}^{0}\left(\widetilde{\mathcal{C}_{b}}\right)$ has four connected components. Over $B$, since $f$ is étale and $f_{*} \mathcal{O}_{\widetilde{\mathcal{C}}_{B}}=\mathcal{O}_{\mathcal{C}_{B}} \oplus \eta_{B}$ is locally free, we can consider the relative norm map [29, §6.5] $\mathrm{Nm}_{B}: \mathrm{Pic}_{\widetilde{\mathcal{C}}_{B}}^{0} \rightarrow \mathrm{Pic}_{\mathcal{C}_{B}}^{0}$, and hence we may consider the inverse images of the zero section of $\mathrm{Pic}_{\mathcal{C}_{B}}^{0} \rightarrow B$ and of the section determined by $\eta_{B}$. By [29, Proposition 6.5.8], on each fiber the norm map is compatible with the norm map associated with the double cover $\widetilde{D}_{b} \rightarrow D_{b}$ between the normalizations of the two curves (cf. (4.1)). Hence, it restricts to a norm map $m: A \times A \rightarrow A$ (notation as in (4.2)), which is nothing but the multiplication map $(a, b) \mapsto a b$. Hence, on every fiber the kernel of the norm map is an extension of the kernel of the norm map of an étale double cover of smooth curves (namely, the normalizations) by ker $m \cong A$. It follows that the inverse image under $\mathrm{Nm}_{B}$ of the zero section has two connected components: the one containing the zero section of $\operatorname{Pic}_{\widetilde{\mathcal{C}}_{B}}^{0}$ and the remaining one. Hence, also the inverse image of the section determined by $\eta_{B}$ has two connected components, and the lemma is proved.

Let

$$
\mathcal{P}_{1}, \quad \mathcal{P}_{2}, \quad \mathcal{P}_{3} \quad \text { and } \quad \mathcal{P}_{4}
$$

be the four connected components of $\operatorname{Fix}\left(-\iota^{*}\right) \subset \operatorname{Pic}_{\widetilde{\mathcal{C}}_{B}}^{0}$, with $\mathcal{P}_{1}$ being the component

$$
\operatorname{Prym}\left(\widetilde{\mathcal{C}}_{B} / \mathcal{C}_{B}\right):=\operatorname{Fix}\left(-\iota^{*}\right)_{0}=\operatorname{Im}\left(1-\iota^{*}\right) \subset \operatorname{Pic}_{\widetilde{\mathcal{C}}_{B}}^{0}
$$

containing the zero section. We will call this component, which is a group scheme of dimension $g-1$ over $B$, the relative generalized Prym variety of $\widetilde{\mathcal{C}}_{B}$ over $\mathcal{C}_{B}$. It is dense in the relative compactified Prym variety, so that its closure $\overline{\mathcal{P}}_{1}$ satisfies

$$
\overline{\mathcal{P}}_{1}=\overline{\operatorname{Prym}}\left(\widetilde{\mathcal{C}}_{B} / \mathcal{C}_{B}\right)=\overline{\operatorname{Im}\left(1-\iota^{*}\right)} .
$$

By restricting $\pi$ to the relative Prym variety, we get a morphism

$$
\nu_{B}: \overline{\operatorname{Prym}}\left(\widetilde{\mathcal{C}}_{B} / \mathcal{C}_{B}\right) \longrightarrow B
$$

whose fiber over a point $b \in B$ corresponding to a double cover between smooth curves $\widetilde{\mathcal{C}_{b}} \rightarrow \mathcal{C}_{b}$ is isomorphic to the usual Prym variety $\operatorname{Prym}\left(\widetilde{\mathcal{C}}_{b} / \mathcal{C}_{b}\right)$. Notice that

$$
\operatorname{dim} \overline{\operatorname{Prym}}\left(\widetilde{\mathcal{C}}_{B} / \mathcal{C}_{B}\right)=\operatorname{dim} B+g-1
$$


We now need to show that this definition is well posed, in the sense that it is compatible with base change. For example, we would like to verify that the fiber over an arbitrary point $b \in B$ of the relative compactified Prym variety is the compactified Prym variety of $\widetilde{\mathcal{C}}_{b}$ over $\mathcal{C}_{b}$.

Proposition 4.10. (1) For every $b \in B$ we have $\overline{\operatorname{Prym}}\left(\widetilde{\mathcal{C}}_{B} / \mathcal{C}_{B}\right)_{\mid b}=\overline{\operatorname{Prym}}\left(\widetilde{\mathcal{C}}_{b} / \mathcal{C}_{b}\right)$.

(2) The base change property holds for arbitrary base change, i.e., for any morphism $B^{\prime} \rightarrow B$, setting $\widetilde{\mathcal{C}}_{B^{\prime}}=\widetilde{\mathcal{C}}_{B} \times{ }_{B} B^{\prime}$ and $\mathcal{C}_{B^{\prime}}=\mathcal{C}_{B} \times{ }_{B} B^{\prime}$, we have

$$
\overline{\operatorname{Prym}}\left(\widetilde{\mathcal{C}}_{B} / \mathcal{C}_{B}\right) \times_{B} B^{\prime}=\overline{\operatorname{Prym}}\left(\widetilde{\mathcal{C}}_{B^{\prime}} / \mathcal{C}_{B^{\prime}}\right) .
$$

(3) The morphism $\nu_{B}: \overline{\operatorname{Prym}}\left(\widetilde{\mathcal{C}}_{B} / \mathcal{C}_{B}\right) \rightarrow B$ is equidimensional.

We claim that it is sufficient to prove (1), since it implies the other two points. Indeed, to show that $(1) \Rightarrow(2)$ we may assume without loss of generality that $B^{\prime}$ is irreducible. Hence $\overline{\operatorname{Prym}}\left(\widetilde{\mathcal{C}}_{B^{\prime}} / \mathcal{C}_{B^{\prime}}\right)$ is irreducible. As it is contained in $\overline{\operatorname{Prym}}\left(\widetilde{\mathcal{C}}_{B} / \mathcal{C}_{B}\right) \times_{B}$ $B^{\prime}$, we only need to show that the latter is also irreducible of the same dimension. By (1) and Remark 4.5, the fiber $\left.\overline{\operatorname{Prym}}\left(\widetilde{\mathcal{C}}_{B} / \mathcal{C}_{B}\right)\right|_{b}$ over any $b \in B$ is irreducible of dimension $g-1$. So, the morphism $\overline{\operatorname{Prym}}\left(\widetilde{\mathcal{C}}_{B} / \mathcal{C}_{B}\right) \times_{B} B^{\prime} \rightarrow B^{\prime}$ is equidimensional and $\overline{\operatorname{Prym}}\left(\widetilde{\mathcal{C}}_{B} / \mathcal{C}_{B}\right) \times_{B} B^{\prime}$ is irreducible of dimension $\operatorname{dim} B^{\prime}+g-1$. The claim is proved.

To prove (1) we first need a few lemmas, as well as the first statement of Proposition 4.20 below. Before stating and proving these lemmas, we need to recall a few properties of compactified Jacobians (cf. [56], [20]).

Let $\Gamma$ be an integral projective curve, with normalization $n: \bar{\Gamma} \rightarrow \Gamma$. Given a rank-1 torsion-free sheaf $F$ on $\Gamma$, there exists [20] a partial normalization

$$
n^{\prime}: \Gamma^{\prime} \longrightarrow \Gamma,
$$

with the property that $\mathcal{E} \operatorname{nd}(F) \cong n_{*}^{\prime} \mathcal{O}_{\Gamma^{\prime}}$ and the rank-1 torsion-free sheaf

$$
F^{\prime}:=\left(n^{\prime}\right)^{*} F / \text { Tors }
$$

on $\Gamma^{\prime}$ satisfies

$$
F=n_{*}^{\prime} F^{\prime}
$$

For later use, we highlight that, given $F$, we can define the curve $\Gamma^{\prime}$ by setting

$$
\Gamma^{\prime}:=\operatorname{Spec}_{\mathcal{O}_{\Gamma}} \mathcal{E} \operatorname{nd}(F) .
$$

We define a local type [20] of rank-1 torsion-free sheaf to be a collection $\left\{M_{p}\right\}_{p \in \operatorname{Sing}(\Gamma)}$ of isomorphism classes of rank-1 torsion-free $\mathcal{O}_{\Gamma, p}$-modules, where $p$ runs in the set of singular point of $\Gamma$.

Two rank-1 torsion-free sheaves $F$ and $G$ on $\Gamma$ are said to be of the same local type if for any $p \in \Gamma$ the localizations $F_{p}$ and $G_{p}$ are isomorphic as $\mathcal{O}_{\Gamma, p}$-modules. 
Proposition 4.11. ([20], [56]) The relative compactified Jacobian $\overline{\mathrm{Jac}}(\Gamma)$ is stratified based on the local type: for every local type $\left\{M_{p}\right\}_{p \in \operatorname{Sing}(\Gamma)}$ there exists a rank-1 torsion-free sheaf $F$ of degree zero with $F_{p} \cong M_{p}$ for every $p$. Furthermore, $\operatorname{Pic}(\Gamma)$ acts transitively on the set of rank-1 torsion-free sheaves of a fixed type $\left\{M_{p}\right\}$, with stabilizer $\operatorname{ker}\left[\operatorname{Pic}^{0}(\Gamma) \rightarrow \operatorname{Pic}^{0}\left(\Gamma^{\prime}\right)\right]$, where $\Gamma^{\prime}:=\operatorname{Spec}_{\mathcal{O}_{\Gamma}} \mathcal{E} \operatorname{nd}(F)$ as above. If $\Gamma$ has planar singularities, then $\overline{\operatorname{Jac}}(\Gamma)$ contains the generalized Jacobian as a dense open subset.

Let us now return to our situation. Our aim is to understand the fixed locus of $\tau$ in $\overline{\operatorname{Jac}}\left(\widetilde{\mathcal{C}}_{B}\right)$. We start by viewing the group homomorphism $\left(1-\iota^{*}\right): \operatorname{Pic}_{\widetilde{\mathcal{C}}_{B}}^{0} \rightarrow \operatorname{Pic}_{\widetilde{\mathcal{C}}_{B}}^{0}$, $L \mapsto L \otimes \iota^{*} L^{\vee}$ as a rational map

$$
\left(1-\iota^{*}\right): \overline{\operatorname{Jac}}\left(\widetilde{\mathcal{C}}_{B}\right)-\rightarrow \overline{\operatorname{Jac}}\left(\widetilde{\mathcal{C}}_{B}\right) .
$$

Let us focus on a neighborhood of the fiber $\overline{\operatorname{Jac}}\left(\widetilde{\mathcal{C}}_{b_{0}}\right)$ over a point $b_{0} \in B$. Let $\left\{x_{1}, \ldots, x_{k}\right\}$ be the singular points of $\mathcal{C}_{b_{0}}$, and let $\left\{p_{1}, \ldots, p_{k}, q_{1}, \ldots, q_{k}\right\}$ be the singular points of $\widetilde{\mathcal{C}}_{b_{0}}$, with $f^{-1}\left(x_{i}\right)=\left\{p_{i}, q_{i}\right\}$. For every subset $I \subset\{1, \ldots, k\}$, we can consider the open subset

$$
V_{I} \subset \overline{\operatorname{Jac}}\left(\widetilde{\mathcal{C}}_{B}\right)
$$

of sheaves that are locally free in a neighborhood of $\left\{p_{i}\right\}_{i \in I}$ and of $\left\{q_{j}\right\}_{j \notin I}$, so that

$$
V=\bigcup_{I} V_{I}
$$

is the open set of sheaves that for every $i$ are locally free at least at one of the two points $p_{i}$ and $q_{i}$.

LEMmA 4.12. The rational map (4.7) is defined in an open neighborhood of $\overline{\operatorname{Jac}}\left(\widetilde{\mathcal{C}}_{B}\right)$ containing $V$.

Proof. It is enough to show that, if $F \in V_{I}$, then $F \otimes \iota^{*} F^{\vee}$ is torsion-free. We only need to check this condition at the singular points $\left\{p_{1}, \ldots, p_{k}, q_{1}, \ldots, q_{k}\right\}$ and, by symmetry, it is enough to check at $p_{i}$, for every $i$. We have $\left(F \otimes \iota^{*} F^{\vee}\right)_{p_{i}}=F_{p_{i}} \otimes F_{q_{i}}^{\vee}$. Since by construction at least one between $F_{p_{i}}$ and $F_{q_{i}}^{\vee}$ is locally free, while the other is torsionfree, their tensor product is torsion-free.

LEMmA 4.13. Given $F \in \operatorname{Fix}(\tau) \subset \overline{\operatorname{Jac}}\left(\widetilde{\mathcal{C}}_{B}\right)$, supported on $\widetilde{\mathcal{C}}_{b_{0}}$, there exists a $G^{\prime} \in V \subset$ $\overline{\operatorname{Jac}}\left(\widetilde{\mathcal{C}}_{B}\right)$ such that the rank-1, $\tau$-invariant, torsion-free sheaf

$$
G:=\left(1-\iota^{*}\right) G^{\prime} \in \overline{\operatorname{Prym}}\left(\widetilde{\mathcal{C}}_{B} / \mathcal{C}_{B}\right)
$$

is of the same local type as $F$. 
Proof. Set $\Gamma:=\widetilde{\mathcal{C}}_{b_{0}}$. Since $\tau(F)=F$, we have

$$
F_{p_{i}}=\left(\iota^{*} F^{\vee}\right)_{p_{i}}=F_{q_{i}}^{\vee},
$$

so that the local type of $F$ is determined by the localizations $F_{p_{1}}, \ldots, F_{p_{k}}$ at only half of the singular points. Consider the local type $\left\{F_{p_{1}}, \ldots, F_{p_{k}}, \mathcal{O}_{q_{1}}, \ldots, \mathcal{O}_{q_{k}}\right\}$, where $\mathcal{O}_{q_{i}}:=\mathcal{O}_{\Gamma, q_{i}}$, and let $G^{\prime}$ be a sheaf in $\overline{\operatorname{Jac}}\left(\widetilde{\mathcal{C}}_{b_{0}}\right)$ with this local type, which exists by Proposition 4.11. With this notation it is clear that

$$
G:=G^{\prime} \otimes \iota^{*} G^{\prime \vee}
$$

is of the same local type as $F$. As $G \in \overline{\operatorname{Im}\left(1-\iota^{*}\right)}=\overline{\operatorname{Prym}}\left(\widetilde{\mathcal{C}}_{B} / \mathcal{C}_{B}\right)$, the lemma is proved.

Set

$$
\Gamma^{\prime}:=\operatorname{Spec}_{\mathcal{O}_{\Gamma}} \mathcal{E} \operatorname{nd}(F),
$$

and let $n^{\prime}: \Gamma^{\prime} \rightarrow \Gamma$ be the natural partial normalization morphism. Since $G$ and $F$ are of the same local type, by Proposition 4.11 we know that there exists an $L \in \operatorname{Pic}_{\widetilde{\mathcal{C}}_{t_{0}}}^{0}$, well defined up to an element of $\operatorname{ker}\left[\left(n^{\prime}\right)^{*}: \operatorname{Pic}^{0}(\Gamma) \rightarrow \operatorname{Pic}^{0}\left(\Gamma^{\prime}\right)\right]$, such that

$$
G=F \otimes L
$$

LEMmA 4.14. Up to changing $L$ by an element of $\operatorname{ker}\left[\operatorname{Pic}^{0}(\Gamma) \rightarrow \operatorname{Pic}^{0}\left(\Gamma^{\prime}\right)\right]$, we may assume that $\tau(L)=L$.

Proof. As $\tau(F)=F$ and $\tau(G)=G$, we have that $M:=L \otimes \tau(L)^{\vee}$ lies in $\operatorname{ker}\left[\operatorname{Pic}^{0}(\Gamma) \rightarrow\right.$ $\left.\operatorname{Pic}^{0}\left(\Gamma^{\prime}\right)\right]$ and satisfies $M^{\vee} \cong \tau(M)$. This last equality implies that $M=\iota^{*}(M)$, and it is not hard to see (cf. for example [59, Lemma 2.8]) that this implies the existence of an $M^{\prime} \in \operatorname{ker}\left[\operatorname{Pic}^{0}(\Gamma) \rightarrow \operatorname{Pic}^{0}\left(\Gamma^{\prime}\right)\right]$ such that $M=M^{\prime} \otimes \iota^{*} M^{\prime}$. From

$$
L=\iota^{*} L^{\vee} \otimes M=\iota^{*} L^{\vee} \otimes M^{\prime} \otimes \iota^{*} M^{\prime},
$$

we deduce that

$$
L^{\prime}:=L \otimes M^{\prime \vee}
$$

satisfies $\iota^{*} L^{\prime \vee}=L^{\prime}$, and since $M^{\prime} \in \operatorname{ker}\left[\operatorname{Pic}^{0}(\Gamma) \rightarrow \operatorname{Pic}^{0}\left(\Gamma^{\prime}\right)\right]$ we still have

$$
G=F \otimes L^{\prime}
$$

Let us now go back to the components $\mathcal{P}_{1}, \ldots, \mathcal{P}_{4}$ defined in (4.6). The morphism

$$
\begin{aligned}
& \mathcal{P}_{1} \otimes \mathcal{P}_{i} \longrightarrow \mathcal{P}_{i}, \\
& (L, M) \longmapsto L \otimes M,
\end{aligned}
$$


extends to a morphism

$$
\begin{aligned}
& \overline{\mathcal{P}}_{1} \otimes \mathcal{P}_{i} \longrightarrow \overline{\mathcal{P}}_{i}, \\
& (G, M) \longmapsto G \otimes M,
\end{aligned}
$$

where, for every $i, \overline{\mathcal{P}}_{i}$ denotes the closure of $\mathcal{P}_{i}$ and hence is an irreducible component of $\operatorname{Fix}(\tau)$. Recall that we defined $\mathcal{P}_{1}$, so that $\overline{\mathcal{P}}_{1}=\overline{\operatorname{Prym}}\left(\widetilde{\mathcal{C}}_{B} / \mathcal{C}_{B}\right)$.

The last element we need is the following lemma.

Lemma 4.15. For $i \neq j$, the closures $\overline{\mathcal{P}}_{i}$ and $\overline{\mathcal{P}}_{j}$ do not intersect.

Proof. This is Corollary 4.21 proved in the next section, which is based only on the smoothness of the relative compactified Jacobian over the versal family of an integral, locally planar curve.

Corollary 4.16. Every irreducible component of $\operatorname{Fix}(\tau) \subset \overline{\operatorname{Jac}}\left(\widetilde{\mathcal{C}}_{B}\right)$ is of the form $\overline{\mathcal{P}}_{i}$ for some $i=1, \ldots, 4$. In particular, in Definition 4.8 we can replace irreducible component with connected component.

Proof. Consider an $F \in \operatorname{Fix}(\tau)$, and let $G$ and $L$ be as in Lemmas 4.13 and 4.14, respectively. Since $\tau(L)=L$ and the fixed locus of $\tau$ on $\operatorname{Pic}_{\widetilde{\mathcal{C}}_{B}}^{0}$ is equal to $\coprod_{i} \mathcal{P}_{i}$, we have $L \in \mathcal{P}_{i}$ for some $i$. Since $G \in \overline{\mathcal{P}}_{1}$, it follows by (4.8) that $F \in \overline{\mathcal{P}}_{i}$.

We finally get to the proof of (1) of Proposition 4.10.

Corollary 4.17. For any $b_{0} \in B$, one has $\overline{\operatorname{Prym}}\left(\widetilde{\mathcal{C}}_{B} / \mathcal{C}_{B}\right)_{\mid b_{0}}=\overline{\operatorname{Prym}}\left(\widetilde{\mathcal{C}}_{b_{0}} / \mathcal{C}_{b_{0}}\right)$.

Proof. We only need to prove that $\overline{\operatorname{Prym}}\left(\widetilde{\widetilde{\mathcal{C}}_{B}} / \mathcal{C}_{B}\right)_{\mid b_{0}} \subset \overline{\operatorname{Prym}}\left(\widetilde{\mathcal{C}_{b_{0}}} / \mathcal{C}_{b_{0}}\right)$, since the reverse inclusion is clear. Consider an $F \in \overline{\operatorname{Prym}}\left(\widetilde{\mathcal{C}}_{B} / \mathcal{C}_{B}\right)_{\mid b_{0}}$. As in Lemmas 4.13 and 4.14, we can find a $\tau$-invariant $L$ and a $G \in \overline{\mathcal{P}}_{1}$, with $G=\left(1-\iota^{*}\right) G^{\prime}$, such that $G=F \otimes L$. By Lemma 4.15 , and the fact that $F \in \overline{\mathcal{P}}_{1}$, we necessarily have $L \in \mathcal{P}_{1}$. As $\mathcal{P}_{1}=\left(1-\iota^{*}\right) \operatorname{Pic}_{\tilde{\mathcal{C}}_{B}}^{0}$, we can find $L^{\prime \prime} \in \operatorname{Pic}_{\tilde{\mathcal{C}}_{t_{0}}}^{0}$ such that $L=\left(1-\iota_{t_{0}}^{*}\right) L^{\prime \prime}$. By construction, $G=\left(1-\iota^{*}\right) G^{\prime}$ and hence

$$
F=\left(1-\iota_{t_{0}}^{*}\right)\left(F^{\prime} \otimes L^{\prime \prime \vee}\right),
$$

from which we see that

$$
F \in \overline{\operatorname{Im}\left(1-\iota_{t_{0}}^{*}\right)}=\overline{\operatorname{Prym}}\left(\widetilde{\mathcal{C}}_{t_{0}} / \mathcal{C}_{t_{0}}\right) .
$$

\subsection{Smoothness results for the relative compactified Prym}

The next step is to study the local structure of the relative compactified Prym variety. This will allows us to formulate a criterion that has to be satisfied by a family $\widetilde{\mathcal{C}}_{B} \rightarrow \mathcal{C}_{B}$ of 
étale double covers of irreducible, locally planar curves in order for the relative compactified Prym variety to be smooth. Since this criterion will be deduced by an analogous criterion for the smoothness of the relative compactified Jacobian, we start by reviewing rapidly, following closely [23], the results we need on this topic.

Let $D$ be a reduced projective curve, with planar singularities. We denote by $\operatorname{Def}(D)$ the deformation functor of the curve $D$ and, for any $p \in D$, we let $\operatorname{Def}\left(D_{p}\right)$ be the deformation functor of the local ring $\mathcal{O}_{D, p}$. For more precise definitions, see $[60, \S 2.4 .1]$. Letting $\operatorname{Sing}(D) \subset D$ denote the singular locus of $D$, set

$$
\operatorname{Def}^{\text {loc }}(D):=\prod_{p \in \operatorname{Sing}(D)} \operatorname{Def}\left(D_{p}\right)
$$

and consider the natural transformation of functors

$$
\Phi: \operatorname{Def}(D) \longrightarrow \operatorname{Def}^{\text {loc }}(D),
$$

which to a deformation of the global curve assigns the induced deformation of local rings at the singular points. Since $D$ is reduced, $\operatorname{Def}(D)$ and $\operatorname{Def}\left(D_{p}\right)$ are unobstructed [60, Example 2.4.9], and hence they admit smooth semi-universal deformations spaces, i.e., there exist a smooth affine scheme $S$, a point $s \in S$, and a transformation of functors

$$
\Psi:(S, s) \longrightarrow \operatorname{Def}(D)
$$

(here, we denote by $(S, s)$ the deformation functor induced by the germ of the complex space), which is smooth and an isomorphism at the level of tangent spaces, and analogously for the $\operatorname{Def}\left(D_{p}\right)$. We say that the semi-universal space is centered at $s \in S$. The tangent spaces to these deformation functors fit into the local to global exact sequence

$$
0 \longrightarrow H^{1}\left(T_{D}\right) \longrightarrow \underbrace{\operatorname{Ext}^{1}\left(\Omega_{D}^{1}, \mathcal{O}_{D}\right)}_{T \operatorname{Def}(D)} \longrightarrow \bigoplus_{p \in \operatorname{Sing}(D)} \underbrace{H^{0}\left(\mathcal{E}_{\mathrm{xt}_{\mathcal{O}_{D, p}}^{1}}\left(\Omega_{D, p}^{1}, \mathcal{O}_{D, p}\right)\right)}_{T \operatorname{Def}\left(D_{p}\right)} \longrightarrow 0,
$$

where $T_{D}:=\mathcal{H} \operatorname{om}\left(\Omega_{D}^{1}, \mathcal{O}_{D}\right)$, and where $H^{1}\left(T_{D}\right)$ is the tangent space to the subfunctor $\operatorname{Def}(D)^{\prime}$ of the deformations of $D$ that are locally trivial. Let $\mathcal{D} \rightarrow S$ be the semiuniversal family for $D$, centered at $s$, and let $\overline{\operatorname{Jac}}(\mathcal{D}) \rightarrow S$ be the relative compactified Jacobian. For any sheaf $F \in \overline{\operatorname{Jac}}(\mathcal{D})$ we can consider the deformation functor of the pair $\operatorname{Def}(F, D)$ and, for any $p \in \operatorname{Sing}(D)$, also of the pair $\operatorname{Def}\left(F_{p}, D_{p}\right)$. By [23, Proposition A3], the $\operatorname{Def}\left(F_{p}, D_{p}\right)$ are smooth functors. As above, there are natural transformations $\Psi^{\prime}:(\overline{\operatorname{Jac}}(\mathcal{D}), F) \rightarrow \operatorname{Def}(F, D)$ and $\Phi^{\prime}: \operatorname{Def}(F, D) \rightarrow \prod \operatorname{Def}\left(F_{p}, D_{p}\right)$. There is a commutative diagram of functors

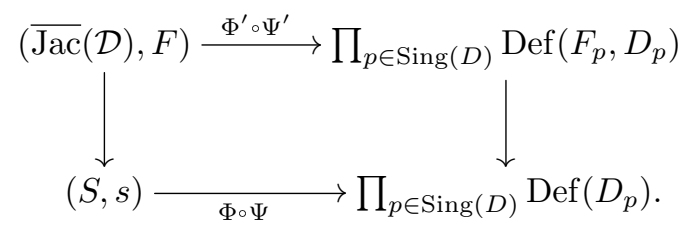


This diagram is not necessarily Cartesian, but the horizontal maps are smooth maps of functors, by the considerations above and by [23, Proposition A1]. In particular, $\overline{\operatorname{Jac}}(\mathcal{D})$ is smooth along $\overline{\operatorname{Jac}}(D)=\overline{\operatorname{Jac}}\left(\mathcal{D}_{s}\right)$. Now, let $\mathcal{D}_{B} \rightarrow B$ be a family of integral locally planar curves, with $\mathcal{D}_{0}=D$ for some $0 \in B$ and with $B$ smooth. There is a morphism $B \rightarrow S$, mapping 0 to $s$, inducing a diagram

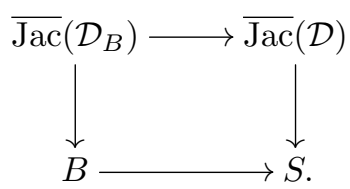

Since the diagram is Cartesian and we are assuming that $B$ is smooth, $\overline{\operatorname{Jac}}\left(\mathcal{D}_{B}\right)$ is smooth at a point $F \in \overline{\operatorname{Jac}}\left(\mathcal{D}_{0}\right)$ if and only if the image of the tangent space $T_{0} B$ in $T_{s} S$ is transversal to the image of $T_{F} \overline{\mathrm{Jac}}(\mathcal{D})$ in $T_{s} S$. Hence, in order to be able to check whether $\overline{\mathrm{Jac}}\left(\mathcal{D}_{B}\right)$ is smooth at a point $F$, we need to understand the image of $T_{F} \overline{\mathrm{Jac}}(\mathcal{D})$ in $T_{s} S$. This is done by analyzing, in the following way, what happens in diagram (4.9) at the level of tangent spaces.

Set $P:=\mathbb{C}[[x, y]]$. As $D$ has locally planar singularities, for any $p_{i} \in \operatorname{Sing}(D)$ there exists $f_{i} \in P$ such that the completion of the local ring $\mathcal{O}_{D, p_{i}}$ is isomorphic to $R_{i}=P / f_{i}$. With this notation, we have

$$
T_{D_{p_{i}}}^{1}\left(=T \operatorname{Def}\left(D, p_{i}\right)\right)=P /\left(f_{i}, \partial_{x} f_{i}, \partial_{y} f_{i}\right)
$$

(note that $T_{D_{p_{i}}}^{1}$ is a vector space of dimension $\tau\left(f_{i}\right)$, see Remark 3.4). For any $i$, let $\bar{R}_{i} \supset R_{i}$ be the normalization of $R_{i}$. We denote by $I_{i} \subset R_{i}$ the conductor ideal, i.e., $I_{i}:=$ $\operatorname{Hom}\left(\bar{R}_{i}, R_{i}\right)$, and we let

$$
V\left(D_{p_{i}}\right) \subset T_{D_{p_{i}}}^{1}
$$

be the image in the Jacobian ring $P /\left(f_{i}, \partial_{x} f_{i}, \partial_{y} f_{i}\right)$ of the conductor ideal. It is a codimension- $\delta_{i}$ subspace, with $\delta_{i}:=\operatorname{dim} \bar{R}_{i} / R_{i}=\operatorname{dim} R_{i} / I_{i}$. Let

$$
V(D) \subset T_{s} S
$$

be the inverse image of $\prod V\left(D_{p_{i}}\right)$ under the tangent map $T_{s} S \rightarrow \prod T_{D_{p_{i}}}^{1}$. It is known that $V(D)$ is the support of the tangent cone to the deformations of $D$ that keep the geometric genus constant, and has codimension in $T_{s} S$ equal to the cogenus $\delta=\sum_{i} \delta_{i}$, i.e. the difference between the arithmetic and the geometric genera of $D$. Let $M$ be a rank-1 torsion-free $R_{i}$-module, viewed as a $P$-module. Recall that $M$ admits a length-1 free resolution

$$
0 \longrightarrow P^{n} \stackrel{\varphi}{\longrightarrow} P^{n} \longrightarrow M \longrightarrow 0
$$


and that the $j$ th Fitting ideal $\mathcal{F}_{j}(M) \subset R_{i}$ of $M$ is the ideal of $R_{i}$ generated by the $(n-j)-$ minors of the matrix $\varphi$. It is independent of the choice of the resolution. For example, the zeroth Fitting ideal is the ideal generated by the local equation $f_{i}$ of the curve at $p_{i}$, i.e., $(\operatorname{det} \varphi)=\left(f_{i}\right) \subset R_{i}$. As for the first Fitting ideal, by [23, Proposition C2] it is the image in $R_{i}$ of the evaluation map $M \times \operatorname{Hom}\left(M, R_{i}\right) \rightarrow R_{i}$. For later use, we highlight the following remark.

Remark 4.18. $\mathcal{F}_{1}(M)=\mathcal{F}_{1}\left(M^{\vee}\right) \subset R_{i}$.

We can now formulate the following key consequence of [23].

Proposition 4.19. ([23]) (a) For any $F \in \overline{\mathrm{Jac}}(D)$, the image of the tangent space $T_{F} \overline{\operatorname{Jac}}(\mathcal{D})$ in $T_{s} S$ contains the space $V(D)$ defined in (4.11).

(b) There exists an $F$ in $\overline{\operatorname{Jac}}(D)$ such that the image is exactly $V(D)$.

(c) $\overline{\operatorname{Jac}}\left(\mathcal{D}_{B}\right)$ is smooth along $\overline{\operatorname{Jac}}(D)$ if and only if the image of $T_{0} B$ in $T_{s} S$ is transversal to $V(D)$.

Proof. By [23, Proposition C1], the image of the tangent space $T \operatorname{Def}\left(F_{p_{i}}, D_{p_{i}}\right)$ in $T \operatorname{Def}\left(p_{i}\right)$ equals the image

$$
W\left(F_{p_{i}}\right) \subset R_{i} /\left(\partial_{x} f_{i}, \partial_{y} f_{i}\right)=T \operatorname{Def}\left(p_{i}\right)
$$

of the first Fitting ideal $\mathcal{F}_{1}\left(F_{i}\right) \subset R_{i}$. By [23, Corollary C3], $\mathcal{F}_{1}\left(F_{i}\right) \supset I_{i}$ and hence the first statement follows from the definition of $V(D)$ and the fact that the tangent map $T_{F} \overline{\operatorname{Jac}}(\mathcal{D}) \rightarrow \prod T \operatorname{Def}\left(F_{p_{i}}, D_{p_{i}}\right)$ is surjective. The third statement follows from the observation made in Remark C4 of [23] that $\mathcal{F}_{1}\left(\bar{R}_{i}\right)=I_{i}$, and from the fact that, by Proposition 4.11 above, there exists an $F \in \overline{\mathrm{Jac}}(D)$ with local type $\left\{\bar{R}_{i}\right\}$. Statement (c) is clear, once we recall that we are assuming that $B$ is smooth and that

$$
\overline{\operatorname{Jac}}\left(D_{B}\right)=\overline{\operatorname{Jac}}(D) \times_{S} B
$$

Let us now get back to our situation and consider

$$
f: \widetilde{C} \longrightarrow C \text { and } \quad \iota: \widetilde{C} \rightarrow \widetilde{C}
$$

an étale double cover of reduced and irreducible curves with planar singularities and the corresponding involution on $\widetilde{C}$. We denote by $\operatorname{Def}(\widetilde{C}, C)$ the deformation functor of the map $\widetilde{C} \rightarrow C[60$, Definition 3.4.1] whose tangent space can be identified by [54] with

$$
T_{C}^{1}=\operatorname{Ext}^{1}\left(\Omega_{C}^{1}, \mathcal{O}_{C}\right)
$$

(that is with $T \operatorname{Def}(C)$ ), viewed as the $\iota$-invariant part of $T_{\widetilde{C}}^{1}=\operatorname{Ext}^{1}\left(\Omega_{\widetilde{C}}^{1}, \mathcal{O}_{\widetilde{C}}\right)$. A semiuniversal family for this functor can be described as follows. Let $\mathcal{C} \rightarrow S$ be a semi-universal 
family for $C$, centered at a point $s \in S$. As in [16], we can consider the finite group scheme $\operatorname{Pic}^{0}(\mathcal{C})[2] \subset \operatorname{Pic}^{0}(\mathcal{C})$ over $S$ which parameterizes 2-torsion line bundles on the curves in $\mathcal{C} \rightarrow S$. Since we are in characteristic zero, the morphism $\operatorname{Pic}^{0}(\mathcal{C})[2] \rightarrow S$ is étale and therefore the natural transformation

$$
\left(\operatorname{Pic}^{0}(\mathcal{C})[2], \eta\right) \longrightarrow \operatorname{Def}(\widetilde{C}, C),
$$

which one can easily check to be smooth, is an isomorphism at the level of tangent spaces. Hence, $\operatorname{Pic}^{0}(\mathcal{C})[2]$ is the base of a semi-universal family for $\operatorname{Def}(\widetilde{C}, C)$. Since $\operatorname{Pic}^{0}(\mathcal{C})[2] \rightarrow S$ is étale, and an isomorphism on tangent spaces, we can replace $S$ by $\operatorname{Pic}^{0}(\mathcal{C})[2]$ so that $(S, s)$ is a semi-universal space for both $\operatorname{Def}(C)$ and $\operatorname{Def}(\widetilde{C}, C)$. In particular, we have a family

$$
f: \widetilde{\mathcal{C}}_{S} \longrightarrow \mathcal{C}_{S}:=\mathcal{C}, \quad \iota: \widetilde{\mathcal{C}}_{S} \longrightarrow \widetilde{\mathcal{C}}_{S}
$$

of étale double covers of integral curves with planar singularities.

We can finally state and prove the smoothness criterion for the relative Prym variety.

THEOREM 4.20. Let the notation be as above.

(1) The relative compactified Prym variety $\overline{\operatorname{Prym}}\left(\widetilde{\mathcal{C}}_{S} / \mathcal{C}_{S}\right)$ over the semi-universal deformation space is smooth.

(2) For any smooth base $B$ and any family of double covers as in (4.5), the relative compactified Prym variety $\overline{\operatorname{Prym}}\left(\widetilde{\mathcal{C}}_{B} / \mathcal{C}_{B}\right)$ is smooth along $\overline{\operatorname{Prym}}\left(\widetilde{\mathcal{C}}_{b} / \mathcal{C}_{b}\right)=\nu_{B}^{-1}(b)$ if and only if the image of the tangent map $T_{b} B \rightarrow T \operatorname{Def}\left(\mathcal{C}_{b}\right)$ of the classifying morphism is transversal to the space $V\left(\mathcal{C}_{b}\right) \subset T \operatorname{Def}\left(\mathcal{C}_{b}\right)$ defined in (4.11).

(3) $\overline{\operatorname{Prym}}\left(\widetilde{\mathcal{C}}_{B} / \mathcal{C}_{B}\right)$ is smooth along $\overline{\operatorname{Prym}}\left(\widetilde{\mathcal{C}}_{b} / \mathcal{C}_{b}\right)=\nu^{-1}(b)$ if and only if $\overline{\operatorname{Jac}}\left(\mathcal{C}_{B}\right)$ is smooth along $\overline{\operatorname{Jac}}\left(\mathcal{C}_{b}\right)=\pi^{-1}(b)$.

Proof. Consider the curve $\widetilde{C}$ and the involution $\iota: \widetilde{C} \rightarrow \widetilde{C}$. A result of Rim (Corollary in [57]) ensures that we may consider a $\iota$-equivariant semi-universal family $\widetilde{\mathcal{C}}_{\widetilde{S}} \rightarrow \widetilde{S}$ for $\widetilde{C}$. By definition, this is a semi-universal family for $\operatorname{Def}(\widetilde{C})$ that has the additional property of admitting compatible actions of $\iota$ on $\widetilde{\mathcal{C}}$ and on $\widetilde{S}$. Let us then consider such a family. We set $T:=\operatorname{Fix}(\iota) \subset \widetilde{S}$ and we denote by $\widetilde{\mathcal{C}}_{T} \rightarrow T$ the restriction of the semi-universal family to $T$. Then

$$
\mathcal{C}_{T}:=\widetilde{\mathcal{C}}_{T} / \iota \longrightarrow T
$$

is a family of integral curves with locally planar singularities. If $\widetilde{S}$ is centered at $s \in \widetilde{S}$, then $\mathcal{C}_{s}=C$, and the tangent space of $T$ at $s$ is the $\iota$-invariant subspace

$$
\left(T_{s} \widetilde{S}\right)^{\iota}=T_{C}^{1}=T \operatorname{Def}(\widetilde{C}, C) .
$$


This shows that $T$ is a semi-universal space for $\operatorname{Def}(\widetilde{C}, C)$. Hence, to prove (1) it is enough to prove that $\overline{\operatorname{Prym}}\left(\widetilde{\mathcal{C}}_{T} / \mathcal{C}_{T}\right)$ is smooth. But this is clear, since $\overline{\operatorname{Prym}}\left(\widetilde{\mathcal{C}}_{T} / \mathcal{C}_{T}\right)$ is by definition just the component of the fixed locus of $\tau$ that contains the zero section of $\overline{\operatorname{Jac}}\left(\widetilde{\mathcal{C}}_{\widetilde{S}}\right)$. Since $\overline{\operatorname{Jac}}\left(\widetilde{\mathcal{C}}_{\widetilde{S}}\right)$ is smooth, so is every component of fixed locus of an involution acting on it.

For item (2), we can reason as in the proof of Proposition 4.19, provided we understand, for any $F \in \overline{\operatorname{Prym}}\left(\widetilde{\mathcal{C}}_{b} / \mathcal{C}_{b}\right)$, the image in $T \operatorname{Def}\left(\mathcal{C}_{b}, \mathcal{C}_{b}\right)$ of the tangent space of the relative compactified Prym variety over a semi-universal deformation space $T$ for $\operatorname{Def}\left(\widetilde{\mathcal{C}}_{b}, \mathcal{C}_{b}\right)$. Indeed, since by Proposition $4.10 \overline{\operatorname{Prym}}\left(\widetilde{\mathcal{C}}_{B} / \mathcal{C}_{B}\right)=\overline{\operatorname{Prym}}\left(\widetilde{\mathcal{C}}_{T} / \mathcal{C}_{T}\right) \times_{T} B$, it is sufficient to prove that, for any $F \in \overline{\operatorname{Prym}}\left(\widetilde{\mathcal{C}}_{b} / \mathcal{C}_{b}\right)$, the image of $T_{F} \overline{\operatorname{Prym}}\left(\widetilde{\mathcal{C}}_{T} / \mathcal{C}_{T}\right)$ in $T \operatorname{Def}\left(\widetilde{\mathcal{C}}_{b}, \mathcal{C}_{b}\right)=T_{\mathcal{C}_{b}}^{1}$ contains the support $V\left(\mathcal{C}_{b}\right)$ of the tangent cone to the equigeneric locus. To see this, we argue as follows. Set $C=\mathcal{C}_{b}$ and $\widetilde{C}=\mathcal{C}_{b}$, and let $\left\{p_{1}, \ldots, p_{k}, q_{1}, \ldots, q_{k}\right\}$ be the singular points of $\widetilde{C}$, with $f^{-1}\left(x_{i}\right)=\left\{p_{i}, q_{i}\right\}$. Consider an $F \in \overline{\operatorname{Prym}}(\widetilde{C} / C)$ and let $\widetilde{S}$ and $T$ be as above. The tangent map

$$
\Xi: T_{F} \overline{\operatorname{Jac}}\left(\widetilde{\mathcal{C}}_{\widetilde{S}}\right) \longrightarrow \prod_{i=1}^{k}\left(T_{\widetilde{C}_{p_{i}}}^{1} \times T_{\widetilde{C}_{q_{i}}}^{1}\right)
$$

is equivariant with respect to the two involutions $\tau$, which acts on $T_{F} \overline{\operatorname{Jac}}\left(\widetilde{\mathcal{C}}_{\widetilde{S}}\right)$ with fixed locus $T_{F} \overline{\operatorname{Prym}}\left(\widetilde{\mathcal{C}}_{T} / \mathcal{C}_{T}\right)$, and $\iota$, which acts on $\prod_{i=1}^{k}\left(T_{\widetilde{C}_{p_{i}}}^{1} \times T_{\widetilde{C}_{q_{i}}}^{1}\right)$ by interchanging $T_{\widetilde{C}_{p_{i}}}^{1}$ with $T_{\widetilde{C}_{q_{i}}}^{1}$ (which are isomorphic since $\iota\left(p_{i}\right)=q_{i}$ ). By item (a) in Proposition 4.19, we know that the image of $\Xi$ is

$$
\prod_{i}\left(W\left(F_{p_{i}}\right) \times W\left(F_{q_{i}}\right)\right)
$$

Here, as in (4.12), $W\left(F_{p_{i}}\right)$ denotes the image of the first Fitting ideal of $F_{p_{i}}$ in $T_{\widetilde{C}_{p_{i}}}^{1}$. Since $\tau(F)=F, F_{q_{i}}=F_{p_{i}}^{\vee}$ and hence, by Remark 4.18, $W\left(F_{p_{i}}\right) \cong W\left(F_{q_{i}}\right)$. It follows that the image of $T_{F} \overline{\operatorname{Prym}}\left(\widetilde{\mathcal{C}}_{T} / \mathcal{C}_{T}\right)$ in

$$
\prod_{i}\left(T \operatorname{Def}\left(\widetilde{C}, p_{i}\right) \times T \operatorname{Def}\left(\widetilde{C}, q_{i}\right)\right)
$$

which is nothing but the $\iota$-invariant subspace of $\operatorname{Im} \Xi$, is equal to the product of diagonals $\prod_{i} \Delta_{W\left(F_{p_{i}}\right)}$. Under the identification $T_{C}^{1}=\left(T_{s} \widetilde{S}\right)^{\iota}$, the subspace $V(C) \subset T_{C}^{1}$ corresponds to the preimage in $T_{s} \widetilde{S}$ of the product $\prod_{i} \Delta_{V\left(\widetilde{C}, p_{i}\right)} \subset \prod_{i}\left(T_{\widetilde{C}_{p_{i}}}^{1} \times T_{\widetilde{C}_{q_{i}}}^{1}\right)$. Since we know that $W\left(F_{p_{i}}\right) \supset V\left(\widetilde{C}, p_{i}\right)$, it follows that $\prod_{i} \Delta_{W\left(F_{p_{i}}\right)} \supset \prod_{i} \Delta_{V\left(\widetilde{C}, p_{i}\right)}$, and hence that the image of $T_{F} \overline{\operatorname{Prym}}\left(\widetilde{\mathcal{C}}_{T} / \mathcal{C}_{T}\right)$ in $T_{C}^{1}$ contains $V(C)$.

The only thing we are left to prove is that there exists an $F \in \overline{\operatorname{Prym}}(\widetilde{C} / C)$ such that this image is exactly $V(C)$. This is done, like in Lemma 4.13, by considering a 
sheaf $F^{\prime} \in \overline{\operatorname{Jac}}(\widetilde{C})$ of local type $\left\{\overline{\mathcal{O}}_{\widetilde{C}, p_{1}}, \ldots, \overline{\mathcal{O}}_{\widetilde{C}, p_{k}}, \mathcal{O}_{\widetilde{C}, p_{1}}, \ldots, \mathcal{O}_{\widetilde{C}, p_{k}}\right\}$, where $\overline{\mathcal{O}}_{\widetilde{C}, p_{i}}$ is the normalization of $\mathcal{O}_{\widetilde{C}, p_{i}}$, and setting

$$
F=F^{\prime} \otimes \iota^{*}\left(F^{\prime}\right)^{\vee} .
$$

As for statement (3), it follows from (2) and Proposition 4.19 (c).

The proof of Proposition 4.20 (1) has the following corollary.

COROLlary 4.21. For any base $T$, the closure $\overline{\mathcal{P}}_{i}$ of the connected components of $\operatorname{Fix}\left(-\iota^{*}\right) \subset \operatorname{Pic}_{\widetilde{\mathcal{C}}_{B}}^{0}$ do not intersect.

Proof. Since $\operatorname{Fix}(\tau) \subset \overline{\operatorname{Jac}}\left(\widetilde{\mathcal{C}}_{\widetilde{S}}\right)$ is smooth, its irreducible components are smooth and disjoint. In particular, the closure in $\overline{\operatorname{Jac}}\left(\widetilde{\mathcal{C}}_{\widetilde{S}}\right)$ of the components of $\operatorname{Fix}\left(-\iota^{*}\right) \subset \operatorname{Pic}_{\widetilde{\mathcal{C}}}^{0}$, which are irreducible components of $\operatorname{Fix}(\tau)$ are smooth and disjoint. If this is true over the semi-universal family, it is a fortiori true that the closures of the $\mathcal{P}_{i}$ 's are disjoint over an arbitrary base.

Corollary 4.22. If $\overline{\operatorname{Prym}}\left(\widetilde{\mathcal{C}}_{B} / \mathcal{C}_{B}\right)$ and $B$ are smooth, then $\nu_{B}: \overline{\operatorname{Prym}}\left(\widetilde{\mathcal{C}}_{B} / \mathcal{C}_{B}\right) \rightarrow B$ is flat.

\section{Descent-From the relative Prym variety to the relative intermediate Jacobian}

In the previous section, we developed a method for associating with any family of double covers of irreducible locally planar curves a relative compactified Prym variety. We now apply these results to the double cover of curves that come up in our situation and get a relative compactified Prym variety $\overline{\mathcal{P}}$. The transversality arguments of $\S 3$ guarantee the smoothness of $\overline{\mathcal{P}}$. Unfortunately, this flat family $\overline{\mathcal{P}}$ (of relative dimension 5) lives over the relative Fano variety $\mathcal{F}$ (or more precisely an open subset of it) and not over the base $B=\left(\mathbb{P}^{5}\right)^{\vee}$, as would be needed in order to compactify the intermediate Jacobian fibration $\mathcal{J}_{U}$. It is therefore necessary to descend $\overline{\mathcal{P}}$ to a family $\overline{\mathcal{J}}$ over $B$ that will give the desired compactification of $\mathcal{J}_{U} \rightarrow U$. This descent argument is the content of this section.

Let $X$ be a general cubic 4-fold (or a general Pfaffian cubic). Let $p: \mathcal{F} \rightarrow B$ be the relative Fano surface, let $\mathcal{F}^{0} / B$ the non-empty open subset of very good lines, in particular not passing through the singular points of the considered hyperplane section. Then $\mathcal{F}^{0} \rightarrow B$ is smooth and, by Proposition 2.10, it is surjective. Let

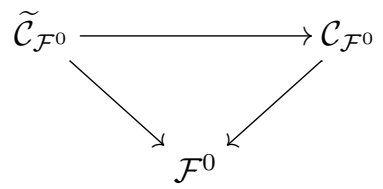


be the associated family of plane quintic curves with their étale double covers. By Proposition 2.10, the curves in the two families are reduced and irreducible. We may therefore apply the results of $\S 4$ and construct the relative compactified Prym variety

$$
\nu: \bar{P}_{\mathcal{F}^{0}}:=\overline{\operatorname{Prym}}\left(\widetilde{\mathcal{C}}_{\mathcal{F}^{0}} / \mathcal{C}_{\mathcal{F}^{0}}\right) \longrightarrow \mathcal{F}^{0} .
$$

Proposition 5.1. The relative compactified Prym $\bar{P}_{\mathcal{F} 0}$ is smooth and the morphism $\nu: \bar{P}_{\mathcal{F}^{0}} \rightarrow \mathcal{F}^{0}$ is flat of relative dimension 5 .

Proof. This follows immediately from Proposition $4.20(2)$ and Corollary 3.9.

As usual, we let $U$ (resp. $U_{1}$ ) be the open subset of $B$ parameterizing hyperplane sections that are smooth (resp. that have a single ordinary node). We set $\mathcal{F}_{U}^{0}=\mathcal{F}^{0} \times_{U} B$ and let $\widetilde{\mathcal{C}}_{\mathcal{F}_{U}^{0}}$ be the restriction of the family of curves. We use the analogous notation for $U_{1}$. For any $t \in B$, and any $\ell \in \mathcal{F}_{t}^{0} \subset F\left(\mathcal{Y}_{t}\right)$, the curve $\widetilde{\mathcal{C}}_{(\ell, t)}$ is the curve of lines in $\mathcal{Y}_{t}$ meeting the line $\ell \subset \mathcal{Y}_{t}$. We let $\mathcal{L}_{U} \subset \widetilde{\mathcal{C}}_{\mathcal{F}_{U}^{0}} \times_{U} \mathcal{Y}_{U}$ be the corresponding universal family of lines of the smooth hyperplane sections. For any $x \in \widetilde{\mathcal{C}}_{(\ell, t)}$, we let $\mathcal{L}_{x}$ be the corresponding line in $\mathcal{Y}_{t}$. There is a relative Abel-Jacobi map

$$
\begin{aligned}
& \Phi_{\mathcal{L}_{U}}: \widetilde{\mathcal{C}}_{\mathcal{F}_{U}^{0}} \longrightarrow \mathcal{J}_{U}, \\
& \widetilde{\mathcal{C}}_{(\ell, t)} \ni x \longmapsto \Phi_{\mathcal{Y}_{t}}\left(\mathcal{L}_{x}-\ell\right) \in \mathcal{J}_{t}=J\left(\mathcal{Y}_{t}\right)
\end{aligned}
$$

inducing a morphism

$$
\Psi: \operatorname{Jac}\left(\widetilde{\mathcal{C}}_{\mathcal{F}_{U}^{0}}\right) \longrightarrow \mathcal{J}_{\mathcal{F}_{U}^{0}}=\mathcal{J}_{U} \times_{U} \mathcal{F}_{U}^{0} .
$$

Since for every $x \in \widetilde{\mathcal{C}}_{(\ell, t)}$ the rational equivalence class of the cycle $\mathcal{L}_{x}+\iota \mathcal{L}_{x}$ in $\mathcal{Y}_{t}$ is constant, the morphism $\Psi$ factors via $(1-\iota) \operatorname{Jac}\left(\widetilde{\mathcal{C}}_{\mathcal{F}_{U}^{0}}\right)=\operatorname{Prym}\left(\widetilde{\mathcal{C}}_{\mathcal{F}_{U}^{0}} / \mathcal{C}_{\mathcal{F}_{U}^{0}}\right)$, thus inducing a morphism from the relative Prym variety

$$
\operatorname{Prym}\left(\widetilde{\mathcal{C}}_{\mathcal{F}_{U}^{0}} / \mathcal{C}_{\mathcal{F}_{U}^{0}}\right) \longrightarrow \mathcal{J}_{\mathcal{F}_{U}^{0}},
$$

which is an isomorphism by a result of Mumford [63]. In particular, over the smooth locus $U$, the relative Prym variety is the pull-back to $\mathcal{F}_{U}^{0}$ of the intermediate Jacobian fibration. The following lemma shows that it is the case also over the locus $U_{1}$ parameterizing 1nodal hyperplane sections.

LEMMA 5.2. The isomorphism (5.1) extends to an isomorphism

$$
\overline{\operatorname{Prym}}\left(\widetilde{\mathcal{C}}_{\mathcal{F}_{U_{1}}^{0}} / \mathcal{C}_{\mathcal{F}_{U_{1}}^{0}}\right) \longrightarrow \mathcal{J}_{\mathcal{F}_{U_{1}}^{0}}=\mathcal{J}_{U_{1}} \times_{U_{1}} \mathcal{F}_{U_{1}}^{0}
$$

In particular, $\mathcal{J}_{U_{1}}$ is smooth. 
Proof. The extensions over the boundary $\mathcal{F}_{U_{1}}^{0} \backslash \mathcal{F}_{U}^{0}$ of the families $\operatorname{Prym}\left(\widetilde{\mathcal{C}}_{\mathcal{F}_{U}^{0}} / \mathcal{C}_{\mathcal{F}_{U}^{0}}\right)$ and $\mathcal{J}_{\mathcal{F}_{U}^{0}}$ to the families $\operatorname{Prym}\left(\widetilde{\mathcal{C}}_{\mathcal{F}_{U_{1}}^{0}} / \mathcal{C}_{\mathcal{F}_{U_{1}}^{0}}\right)$ and $\mathcal{J}_{\mathcal{F}_{U_{1}}^{0}}^{\circ}$, respectively, of semi-abelian varieties are determined by the monodromy of the local systems over $\mathcal{F}_{U}^{0}$ : indeed, if $\mathcal{H}_{\text {Prym }}^{0,1}$ and $\mathcal{H}_{\mathcal{J}}^{0,1}$ are the Hodge bundles of the families $\nu: \operatorname{Prym}\left(\widetilde{\mathcal{C}}_{\mathcal{F}_{U}^{0}} / \mathcal{C}_{\mathcal{F}_{U}^{0}}\right) \rightarrow \mathcal{F}_{U}^{0}$ and $\varrho: \mathcal{J}_{\mathcal{F}_{U}^{0}} \rightarrow \mathcal{F}_{U}^{0}$, respectively, then the two families of semi-abelian varieties (or rather their sheaves of local sections $) \operatorname{Prym}\left(\widetilde{\mathcal{C}}_{\mathcal{F}_{U_{1}}^{0}} / \mathcal{C}_{\mathcal{F}_{U_{1}}^{0}}\right)$ and $\mathcal{J}_{\mathcal{F}_{U_{1}}^{0}}^{\circ}$ are given, respectively, by

$$
\overline{\mathcal{H}}_{\text {Prym }}^{0,1} / j_{*} R^{1} \nu_{*} \mathbb{Z}, \quad \text { and } \quad \overline{\mathcal{H}}_{\mathcal{J}}^{0,1} / j_{*} R^{1} \varrho_{*} \mathbb{Z} \text {. }
$$

Here $\overline{\mathcal{H}}_{\text {Prym }}^{0,1}$ and $\overline{\mathcal{H}}_{\mathcal{J}}^{0,1}$ are the canonical extensions of the Hodge bundle across $\mathcal{F}_{U_{1}}^{0} \backslash \mathcal{F}_{U}^{0}$, which is smooth, and $j: \mathcal{F}_{U}^{0} \rightarrow \mathcal{F}_{U_{1}}^{0}$ is the inclusion. Since the two families are isomorphic over $\mathcal{F}_{U}^{0}$ by $(5.1)$, so are the corresponding local systems and Hodge bundles, and hence so are the canonical extensions. We thus get an isomorphism

$$
\operatorname{Prym}\left(\widetilde{\mathcal{C}}_{\mathcal{F}_{U_{1}}^{0}} / \mathcal{C}_{\mathcal{F}_{U_{1}}^{0}}\right) \cong \mathcal{J}_{\mathcal{F}_{U_{1}}^{0}}^{\circ}=\mathcal{J}_{U_{1}}^{\circ} \times_{U_{1}} \mathcal{F}_{U_{1}}^{0}
$$

The fact that this isomorphism extends to the Mumford compactifications, where along the boundary the $\mathbb{C}^{*}$-bundles are replaced with the corresponding $\mathbb{P}^{1}$-bundle with the sections 0 and $\infty$ glued via a translation, follows from the fact that the Mumford compactification is canonical. We thus get the desired isomorphism (5.2).

From now on, we will use the following notation (justified by Lemma 5.2): For any morphism $f: M \rightarrow \mathcal{F}^{0}$ with induced morphism $f^{\prime}=p \circ f: M \rightarrow B$, we will denote by $\mathcal{J}_{M}$ the pull-back $\bar{P}_{\mathcal{F}^{0}} \times_{\mathcal{F}^{0}} M$ and by $\pi_{M}: \mathcal{J}_{M} \rightarrow M$ the second projection. Over $M_{1}:=\left(f^{\prime}\right)^{-1}\left(U_{1}\right)$, one has $\left.\mathcal{J}_{M}\right|_{M_{1}}=\mathcal{J}_{U_{1}} \times_{U_{1}} M_{1}$ by Lemma 5.2 . The aim of this section is to show a result extending in some sense Lemma 5.2 over the whole of $B$, that is, to construct a projective compactification $\overline{\mathcal{J}}$ of $\mathcal{J}_{U_{1}}$ that is flat over $B$, whose pull-back to $\mathcal{F}^{0}$ will be isomorphic to $\bar{P}_{\mathcal{F}^{0}}=\mathcal{J}_{\mathcal{F}^{0}}$. Then $\overline{\mathcal{J}}$ will be clearly smooth. This is a descent problem which will use the following Proposition 5.3. The morphism $\pi_{U_{1}}: \mathcal{J}_{U_{1}} \rightarrow U_{1}$ is projective. In fact, there is a canonical Theta divisor $\Theta_{1} \subset \mathcal{J}_{U_{1}}$ defined as the Zariski closure in $\mathcal{J}_{U_{1}}$ of the canonically defined divisor $\Theta \subset \mathcal{J}$ (see Lemma 5.4 for more detail). Using Lemma 5.2, we get by pull-back a divisor $\widetilde{\Theta}_{1}$ on $\mathcal{J}_{U_{1}} \times_{U_{1}} \mathcal{F}_{U_{1}}^{0}=\mathcal{J}_{\mathcal{F}_{U_{1}}^{0}}$, and as the morphism $\pi_{\mathcal{F}^{0}}: \mathcal{J}_{\mathcal{F}^{0}} \rightarrow \mathcal{F}^{0}$ is flat, $\mathcal{J}_{\mathcal{F}_{U_{1}}^{0}} \subset \mathcal{J}_{\mathcal{F}^{0}}$ has a complement of codimension $\geqslant 2$, and thus $\widetilde{\Theta}_{1}$ extends uniquely to a divisor $\widetilde{\Theta}_{1}$ on $\mathcal{J}_{\mathcal{F}^{0}}$.

Proposition 5.3. The divisor $\overline{\widetilde{\Theta}}_{1}$ on $\mathcal{J}_{\mathcal{F}^{0}}$ is $\pi_{\mathcal{F}^{0} \text {-ample. }}$

The proof will use several lemmas. We first recall the following lemma.

Lemma 5.4. For any smooth cubic 3-fold $Y$, there is a canonically defined Theta divisor $\Theta_{Y} \subset J(Y)$ which is invariant under the involution -1 . 
Proof. The divisor $\Theta_{Y}$ is defined as follows: Consider the family $\mathcal{C} \rightarrow \mathcal{H}$ of rational cubic curves in $Y$. We have the Abel-Jacobi map

$$
\begin{aligned}
& \Phi_{\mathcal{C}}: \mathcal{H} \longrightarrow J(Y), \\
& s \longmapsto \Phi_{Y}\left(\mathcal{C}_{s}-h^{2}\right),
\end{aligned}
$$

where $h=c_{1}\left(\mathcal{O}_{Y}(1)\right) \in \mathrm{CH}^{2}(Y)$, so that $\mathcal{C}_{s}-h^{2}$ is homologous to zero in $Y$. The fact that the image of this map is a Theta divisor in $J(Y)$ is proved in $[19, \S 13]$ (see also [30]). The fact that it is a $(-1)$-invariant divisor in $J(Y)$ follows from the following observation $([30])$.

Sublemma 5.5. Let $C \subset Y$ be a general cubic rational normal curve in a smooth cubic 3-fold. Then, there exist two lines $L, L^{\prime} \subset Y$ such that $C$ is rationally equivalent in $Y$ to $c_{1}\left(\mathcal{O}_{Y}(1)\right)^{2}+L-L^{\prime}$. Conversely, for two general lines $L$ and $L^{\prime}$ on $Y$, we have that $c_{1}\left(\mathcal{O}_{Y}(1)\right)^{2}+L-L^{\prime}$ is rationally equivalent in $Y$ to a smooth rational cubic curve $C$.

Proof. The curve $C$ generates a $\mathbb{P}^{3}$ which intersects $Y$ in a cubic surface $S \subset Y$, which is smooth because $C$ is generic. The linear system $\left|\mathcal{O}_{S}(C)\right|$ is a $\mathbb{P}^{2}$ which provides a birational map $\phi: S \rightarrow \mathbb{P}^{2}$, contracting six lines $L_{i}$ in $S$ to points $p_{i}$. The curve $C$ belongs to the linear system $\left|\phi^{*} \mathcal{O}_{\mathbb{P}^{2}}(1)\right|$. Choose a line $L$ contracted to a point $p$, and consider the proper transform $L^{\prime}$ in $S$ of a conic passing through all points $p_{i}$ except $p$. Then $L^{\prime}$ is a line in $S$ which belongs to the linear system $\left|\phi^{*} \mathcal{O}_{\mathbb{P}^{2}}(2)\left(-\sum_{p_{i} \neq p} L_{i}\right)\right|$ and thus $L^{\prime}-L$ is rationally equivalent in $S$ to $\phi^{*} \mathcal{O}_{\mathbb{P}^{2}}(2)\left(-\sum_{i} L_{i}\right)$. But $K_{S}=-\left.h\right|_{S}=\phi^{*} \mathcal{O}_{\mathbb{P}^{2}}(-3)\left(\sum_{i} L_{i}\right)$ (with $h=c_{1}\left(\mathcal{O}_{Y}(1)\right)$ ), hence we get

$$
-\left.h\right|_{S}+\phi^{*} \mathcal{O}_{\mathbb{P}^{2}}(1)=L-L^{\prime}
$$

in $\mathrm{CH}^{1}(S)$, and thus $C=h^{2}+L-L^{\prime}$ in $\mathrm{CH}^{2}(Y)$. Conversely, if $L$ and $L^{\prime}$ are two lines in $Y$, the $\mathbb{P}^{3}=P_{3}$ generated by $L$ and $L^{\prime}$ intersects $Y$ along a smooth cubic surface, and for a given point $x \in L$, the plane $\left\langle L^{\prime}, x\right\rangle \subset P_{3}$ intersects $Y$ along the union of $L^{\prime}$ and a conic $C^{\prime}$ meeting $L$ at $x$. The curve $L \cup C^{\prime}$ is a reducible rational cubic curve $C$ in $S$ (which deforms to a smooth rational cubic curve).

It follows that the divisor $\operatorname{Im} \Phi_{\mathcal{C}}$ (or rather 6 times this divisor) is also equal to the image in $J(Y)$ of the difference map

$$
\begin{array}{r}
F(Y) \times F(Y) \longrightarrow J(Y), \\
\left(l_{1}, l_{2}\right) \longmapsto l_{1}-l_{2} .
\end{array}
$$

Thus it is invariant under the involution $(-1)$ of $J\left(Y_{t}\right)$. 
Coming back to our family $\pi_{U}: \mathcal{J}_{U} \rightarrow U$, on which the (-1)-involution acts over $U$, we constructed from Lemma 5.4 a canonical Theta divisor $\Theta \subset \mathcal{J}_{U}$ which is -1-invariant, with Zariski closure $\Theta_{1} \subset \mathcal{J}_{U_{1}}$. We now have the following lemma.

LEMma 5.6. For any dominant morphism $f^{\prime}: M_{1} \rightarrow U_{1}, M_{1}$ irreducible, with canonical lift $f: \mathcal{J}_{M_{1}} \rightarrow \mathcal{J}_{U_{1}}$, the (-1)-invariant part of Pic $\mathcal{J}_{M_{1}} / M_{1}$, where $\mathcal{J}_{M_{1}}:=\mathcal{J} \times_{U_{1}} M_{1}$, is generated modulo torsion by $f^{*} \Theta_{1}$.

Proof. The relative Picard group Pic $\mathcal{J}_{M_{1}} / M_{1}:=\operatorname{Pic} \mathcal{J}_{M_{1}} / \pi_{M_{1}}^{*}$ Pic $M_{1}$ injects in the Picard group of the fiber $J\left(Y_{t}\right)$, where $t \in M$ is very general, because the fibers of the map $\pi_{M}$ (which are also the fibers of the map $\pi_{U_{1}}: \mathcal{J}_{U_{1}} \rightarrow U_{1}$ ) are all reduced and irreducible. In particular, it injects into Pic $\mathcal{J}_{M_{U}} / M_{U}$, where $M_{U}:=f^{\prime-1}(U) \subset M_{1}$. We are thus reduced to proving that the $(-1)$-invariant part of Pic $J\left(Y_{t}\right)$ is, modulo torsion, generated by $\Theta_{Y_{t}}$ for $t$ very general in $U$. However, modulo torsion, the $(-1)$-invariant part of $\operatorname{Pic} J\left(Y_{t}\right)$ is isomorphic to the Néron-Severi group of $J\left(Y_{t}\right)$. Finally, recall that we have a canonical isomorphism $H^{3}\left(Y_{t}, \mathbb{Q}\right) \cong H^{1}\left(J\left(Y_{t}\right), \mathbb{Q}\right)$ which provides more generally an isomorphism of local systems over $U$ :

$$
R^{3} u_{*} \mathbb{Q} \cong R^{1} \pi_{U *} \mathbb{Q}
$$

For the local system on the left, the corresponding monodromy group

$$
\operatorname{Im}\left(\varrho: \pi_{1}(U, t) \longrightarrow \operatorname{Aut} H^{3}\left(Y_{t}, \mathbb{Z}\right)\right)
$$

is the full symplectic group of the intersection pairing by Picard-Lefschetz theory [10]. Hence, the same is true for the local system on the right. As $M_{1}$ is irreducible and the morphism $f^{\prime}: M_{1} \rightarrow U_{1}$ is dominating, the image of the morphism

$$
\left(f_{U}^{\prime}\right)_{*}: \pi_{1}\left(M_{U}, m_{t}\right) \longrightarrow \pi_{1}(U, t)
$$

where $f_{U}^{\prime}: M_{U} \rightarrow U$ is the restriction of $f^{\prime}$ and $f_{U}^{\prime}\left(m_{t}\right)=t$, is a subgroup of finite index in $\pi_{1}(U, t)$. Thus, the monodromy group of the family $\mathcal{J}_{M_{U}} \rightarrow M_{U}$ acts via a subgroup of finite index of $\operatorname{Sp}\left(H^{1}\left(\mathcal{J}_{m_{t}}, \mathbb{Z}\right)\right)$ on the cohomology of $\mathcal{J}_{m_{t}}$. On the other hand, it is a general fact that the monodromy group acts on $H^{2}\left(J\left(Y_{m_{t}}\right), \mathbb{Q}\right)=\bigwedge^{2} H^{1}\left(J\left(Y_{m_{t}}\right), \mathbb{Q}\right)$ by preserving (for very general $t$ ) the Néron-Severi group of $J\left(Y_{t}\right)$ and with finite orbits on $\mathrm{NS}\left(J\left(Y_{t}\right)\right)_{\mathbb{Q}}$. The only elements of $\bigwedge^{2} H^{1}\left(J\left(Y_{m_{t}}\right), \mathbb{Z}\right)$ which have finite orbit under $\operatorname{Sp}\left(H^{1}\left(\mathcal{J}_{m_{t}}, \mathbb{Z}\right)\right)$ are the multiples of the class $\left[\Theta_{t}\right]$, and we conclude that

$$
\mathrm{NS}\left(J\left(Y_{t}\right)\right)_{\mathbb{Q}}=\mathbb{Q}\left[\Theta_{Y_{t}}\right]
$$

Proof of Proposition 5.3. The morphism $\pi_{\mathcal{F}^{0}}: \mathcal{J}_{\mathcal{F}^{0}} \rightarrow \mathcal{F}^{0}$ is projective. Next we recall from Lemma 4.7 that the involution $(-1)$ acting on $\mathcal{J}_{U_{1}}$, hence on $\mathcal{J}_{\mathcal{F}_{U_{1}}^{0}}$ by Lemma 5.2 , 
extends to an involution acting on $\mathcal{J}_{\mathcal{F}^{0}}$ over $\mathcal{F}^{0}$ : it can be defined as the involution $\mathcal{F} \mapsto \mathcal{F}^{\vee}$ acting on reflexive sheaves on $\widetilde{C}_{l, Y}$, where for any point $([l],[Y]) \in \mathcal{F}^{0}$, the curve $\widetilde{C}_{l, Y}$ is the incidence curve of the line $l \in F(Y)$ (cf. Lemma 4.7). Notice that, since the relative compactified Prym variety is defined as a component of the fixed locus of $-\iota^{*}$, where $\iota: \widetilde{C}_{l, Y} \rightarrow \widetilde{C}_{l, Y}$ is the usual natural involution, we have $(-1)=\iota^{*}$ on $\mathcal{J}_{\mathcal{F}_{U_{1}}^{0}}$. It follows

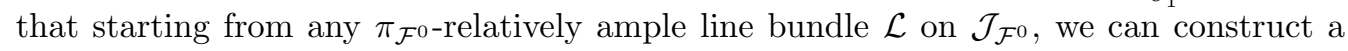

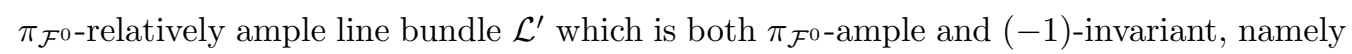

$$
\mathcal{L}^{\prime}=\mathcal{L} \otimes(-1)^{*} \mathcal{L}
$$

We now apply Lemma 5.6 to the natural morphism $f^{\prime}=\left.p\right|_{\mathcal{F}_{U_{1}}^{0}}: \mathcal{F}_{U_{1}}^{0} \rightarrow U_{1}$ and the $(-1)$ invariant line bundle $\left.\mathcal{L}^{\prime}\right|_{\mathcal{J}_{\mathcal{F}_{1}^{0}}^{0}}$. It says that, up to replacing $\mathcal{L}^{\prime}$ by a multiple, we have

$$
\left.\mathcal{L}^{\prime}\right|_{\mathcal{J}_{\mathcal{F}_{U_{1}}^{0}}}=f^{*} \mathcal{O}\left(d \Theta_{1}\right) \otimes \pi_{\mathcal{F}_{U_{1}}^{0}}^{*} \mathcal{N}_{1}
$$

for some integer $d$, where $\mathcal{N}_{1}$ is a line bundle on $\mathcal{F}_{U_{1}}^{0}$. Note that $\operatorname{Pic} \mathcal{F}_{U_{1}}^{0}=\operatorname{Pic} \mathcal{F}^{0}$, because $\mathcal{F}^{0}$ is smooth and $\operatorname{codim}\left(\mathcal{F}^{0} \backslash \mathcal{F}_{U_{1}}^{0} \subset \mathcal{F}^{0}\right) \geqslant 2$. For the same reason, $\operatorname{Pic} \mathcal{J}_{\mathcal{F}_{U_{1}}^{0}}=\operatorname{Pic} \mathcal{J}_{\mathcal{F}^{0}}$, because $\pi_{\mathcal{F}^{0}}$ is flat. Hence, also $\operatorname{codim}\left(\mathcal{J}_{\mathcal{F}^{0}} \backslash \mathcal{J}_{\mathcal{F}_{U_{1}}^{0}} \subset \mathcal{J}_{\mathcal{F}^{0}}\right) \geqslant 2$ and $\mathcal{J}_{\mathcal{F}^{0}}$ is smooth. Thus, the line bundle $\mathcal{N}_{1}$ extends to a unique line bundle $\mathcal{N}$ on $\mathcal{F}^{0}$ and (5.3) is true as well over $\mathcal{J}_{\mathcal{F}^{0}}$, proving that

$$
\mathcal{L}^{\prime}=\mathcal{O}\left(d \widetilde{\widetilde{\Theta}}_{1}\right) \otimes \pi_{\mathcal{F}^{0}}^{*} \mathcal{N}
$$

As $\mathcal{L}^{\prime}$ is $\pi_{\mathcal{F}^{0}}$ ample, so is $\widetilde{\widetilde{\Theta}}_{1}$ by $(5.4)$.

Using the results above and Proposition 5.1, we now prove the following result (see main theorem in the introduction).

THEOREM 5.7. Let $j_{1}: U_{1} \rightarrow B$ be the inclusion. Then

(i) For any sufficiently large integer $d>0$, the sheaf of algebras

$$
\mathcal{E}^{*}:=R^{0} j_{1 *}\left(\bigoplus_{k \geqslant 0} R^{0} \pi_{U_{1} *} \mathcal{O}_{\mathcal{J}_{U_{1}}}\left(k d \Theta_{1}\right)\right)
$$

is a sheaf of $\mathcal{O}_{B}$-algebras of finite type, and each summand $R^{0} j_{1 *}\left(R^{0} \pi_{U_{1} *} \mathcal{O}_{\mathcal{J}_{U_{1}}}\left(k d \Theta_{1}\right)\right)$ is a locally free coherent sheaf on $B$.

(ii) The variety $\overline{\mathcal{J}}:=\operatorname{Proj}\left(\mathcal{E}^{*}\right) \rightarrow B$ is a smooth projective compactification of $\mathcal{J}_{U_{1}}$.

(iii) The variety $\overline{\mathcal{J}}$ is irreducible hyper-Kähler.

Proof. (i), (ii) It suffices to prove the existence of $d$ locally in the Zariski topology. For any $b \in B$ there exists, by Corollary 2.11, a very good line $l_{b} \in Y_{b}$. As the family $\mathcal{F}^{0} \rightarrow B$ of very good lines in the fibers of $u$ is smooth over $B$, we may assume, up 
to an étale base change $f^{\prime}: M \rightarrow B, m \mapsto b$, that there is a section $f: M \rightarrow \mathcal{F}_{M}, m \mapsto l_{m}$. Furthermore, the conclusion of Corollary 3.9 holds, so that the corresponding family of plane quintic curves $C_{l_{m}}$ induces a versal deformation of $\operatorname{Sing}\left(C_{l_{m}}\right)$. We can then apply the results of $\S 4$, and especially Theorem 4.20 , which provides a smooth projective flat compactification $\pi_{M}: \mathcal{J}_{M} \rightarrow M$ of $\mathcal{J}_{M_{1}}:=\mathcal{J}_{U_{1}} \times_{U_{1}} M_{1}$, where $M_{1}=f^{\prime-1}\left(U_{1}\right) \subset M$. If $\mathcal{L}$ is a $\pi_{M}$-relatively ample line bundle on $\mathcal{J}_{M}$, for some $l_{0}$ large enough, $R^{i} \pi_{M *} \mathcal{L}^{\otimes n}=0$ for $n \geqslant l_{0}, i>0$, and thus we conclude by flatness of $\pi_{M}$ that:

(a) $R^{0} \pi_{M *} \mathcal{L}^{\otimes n}$ is locally free for $n \geqslant d_{0}$;

(b) $\bigoplus_{k} R^{0} \pi_{M *} \mathcal{L}^{\otimes k d_{0}}$ is a sheaf of finitely generated $\mathcal{O}_{M^{-}}$-algebras;

(c) the smooth variety $\mathcal{J}_{M}$ is isomorphic over $M$ to $\operatorname{Proj}\left(\bigoplus_{k} R^{0} \pi_{M *} \mathcal{L}^{\otimes k d_{0}}\right)$.

Next, let $j_{M_{1}}: M_{1} \rightarrow M$ be the inclusion map. As $M \backslash M_{1}$ has codimension $\geqslant 2$, and $\pi_{M}$ is flat, $\mathcal{J}_{M} \backslash \mathcal{J}_{M_{1}}$ also has codimension $\geqslant 2$. As $M$ and $\mathcal{J}_{M}$ are smooth, we conclude that

$$
R^{0} j_{M_{1} *}\left(R^{0} \pi_{M_{1} *}\left(\left.\mathcal{L}\right|_{\mathcal{J}_{M_{1}}} ^{\otimes k}\right)\right)=R^{0} \pi_{M *}\left(\mathcal{L}^{\otimes k}\right) .
$$

We now assume that $\mathcal{L}$ is $(-1)$-invariant, so $\mathcal{L}=\mathcal{L}^{\prime}$ satisfies (5.4). Up to shrinking $M$, the line bundle $\mathcal{N}$ appearing in (5.4) is trivial on $M$, so that $\left.\mathcal{L}\right|_{\mathcal{J}_{M_{1}}}=f_{1}^{*} \mathcal{O}\left(d^{\prime} \Theta_{1}\right)$ for some integer $d^{\prime}$, where $f_{1}: \mathcal{J}_{M_{1}} \rightarrow \mathcal{J}_{U_{1}}$ is the natural map over $\left.f^{\prime}\right|_{M_{1}} / M_{1} \rightarrow U_{1}$. Thus, (a)-(c) above and (5.5) prove (i) and (ii) after pull-back to $M$. In other words, we proved that (i) and (ii) are true étale locally on $B$, that is, after étale base changes $f^{\prime}: M \rightarrow B$ of small Zariski open sets of $B$ covering $B$. This clearly implies (i) and (ii), for example because an étale base change is a local isomorphism in the analytic topology, and (i) and (ii) are local statements in the analytic topology.

(iii) We know by Proposition 1.9 that $\mathcal{J}_{U_{1}}$ has a non-degenerate holomorphic 2-form, which extends to a non-degenerate holomorphic 2 -form on $\overline{\mathcal{J}}$, as $\operatorname{codim}\left(\overline{\mathcal{J}} \backslash \mathcal{J}_{U_{1}} \subset \overline{\mathcal{J}}\right) \geqslant 2$. What remains to be done is to prove that $\overline{\mathcal{J}}$ is irreducible hyper-Kähler. We have the following lemma.

LEMMA 5.8. The holomorphic 2-forms on any finite étale cover $\widetilde{\mathcal{J}}$ of $\overline{\mathcal{J}}$ are multiples of the form $\sigma$ coming from $X$.

Proof. The variety $\overline{\mathcal{J}}$ contains the Zariski closure of the Theta divisor $\Theta \subset \mathcal{J}$ which is birational to a $\mathbb{P}^{1}$-bundle over the Lehn-Lehn-Sorger-van Straten variety $F_{3}(X)$. Indeed, recall from [42] that $F_{3}(X)$ parameterizes birationally nets $|D|$ of rational cubic curves on cubic surfaces $S \subset X$. Consider the $\mathbb{P}^{1}$-bundle $\mathbb{P} \rightarrow F_{3}(X)$ having as fiber over $(S, D)$ the $\mathbb{P}^{1}$ of hyperplanes in $\mathbb{P}^{5}$ containing $S$. Then $\mathbb{P}$ admits a morphism to $\left(\mathbb{P}^{5}\right)^{*}$ whose fiber over $H_{Y}$ parameterizes the nets of cubic rational curves on cubic surfaces in $Y$. We already mentioned that, via the map

$$
|D| \longmapsto \Phi_{X}\left(D-h^{2}\right) \in J(Y),
$$


the set of such nets dominates (in fact, is birational to) the Theta divisor of $J(Y)$. This

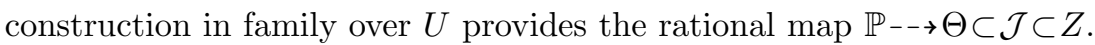

The proof of Lemma 5.8 is now immediate: The variety $F_{3}(X)$ is simply connected (it is a deformation of $S^{[4]}$ for some K3 surface $S$; see [1]), and hence $\mathbb{P}$ is simply connected, so the rational map $\mathbb{P}-\rightarrow \overline{\mathcal{J}}$ constructed above lifts to a rational map $\mathbb{P}-\rightarrow \widetilde{\mathcal{J}}$ for any finite étale cover $\widetilde{\overline{\mathcal{J}}}$ of $\overline{\mathcal{J}}$. As $F_{3}(X)$ is an irreducible hyper-Kähler manifold and $\mathbb{P} \rightarrow F_{3}(X)$ is a $\mathbb{P}^{1}$-bundle, the holomorphic 2 -forms on $\mathbb{P}$ are all multiples of the restriction of $\sigma_{\bar{J}}$. It thus only suffices to show that, if $\alpha$ is a holomorphic 2-form on $\widetilde{\mathcal{J}}$ which vanishes on the image $\Theta$ of $\mathbb{P}$, then $\alpha=0$. That follows however immediately from the fact that, fiberwise, as $\Theta_{t} \subset \widetilde{\mathcal{J}}_{t}$ is an ample divisor, the restriction map

$$
H^{0}\left(\widetilde{\mathcal{J}}_{t}, \Omega_{\widetilde{\mathcal{J}}_{t}}^{i}\right) \longrightarrow H^{0}\left(\Theta_{t}^{0}, \Omega_{\Theta_{t}^{0}}^{i}\right)
$$

is injective for $t \in U$, and $0 \leqslant i \leqslant 2$, where $\Theta_{t}^{0}$ is the smooth locus of $\Theta_{t}$.

By the Beauville-Bogomolov decomposition theorem [9], Lemma 5.8 implies that $\overline{\mathcal{J}}$ is irreducible hyper-Kähler.

\section{Construction of a birational map in the Pfaffian case}

We have established in $\S 3$ that a general Pfaffian cubic 4-fold satisfies the same versality statements as a general cubic, and thus the relative intermediate Jacobian associated with a general Pfaffian cubic has a smooth projective hyper-Kähler compactification $\overline{\mathcal{J}}$. The purpose of this section is to establish that this compactification $\overline{\mathcal{J}}$ is birational to an OG10 hyper-Kähler manifold, and thus by Huybrechts' result [33] (two birationally equivalent HK manifolds are deformation equivalent) is deformation equivalent to OG10. This completes the proof of the main theorem (stated in the introduction).

What is used about Pfaffian cubic 4-folds $X$ is that they have associated (cf. [13]) degree-14 K3 surfaces $\Sigma$. We will prove below that birationally the relative intermediate Jacobian fibration $\overline{\mathcal{J}}$ associated with a Pfaffian cubic $X$ can be interpreted as a moduli space of sheaves on $\Sigma$, linking in with the original construction of OG10 by O'Grady [50].

Remark 6.1. Of course, the same approach can be applied to other classes of cubic 4-folds for which there is an associated K3 surface (in the sense of Hassett [32]). In particular, as mentioned in the introduction, the relative intermediate Jacobian fibration can be seen to be related to the OG10 construction in the case of nodal cubic 4-folds (cf. Hwang-Nagai [35]), or in the case of degenerations to the chordal cubic 4-folds (cf. O'Grady-Rapagnetta). In fact, in those cases the geometry of relating the relative intermediate Jacobian fibration to sheaves on the associated K3 is easier than in the 
Pfaffian case. However, the problem is that our construction will not lead to a smooth compactification $\overline{\mathcal{J}}$ (and thus Huybrechts' result does not suffices). In other words, it is essential for our proof of deformation equivalence to OG10 that the general Pfaffian cubic satisfies both: it has an associated K3 and it behaves similarly to a general cubic from the perspective of good lines (see $\S 3$, especially Corollary 3.7 and Lemma 3.12). $\left({ }^{2}\right.$ )

\subsection{The Pfaffian case-Statement of the main result and strategy}

Let $X$ be a Pfaffian cubic 4-fold. This means that there is a 6 -dimensional vector space $V_{6}$ and a 6-dimensional vector space $W_{6} \subset \bigwedge^{2} V_{6}^{*}$ such that $X \subset \mathbb{P}\left(W_{6}\right)$ is the Pfaffian cubic hypersurface defined by the Pfaffian equation $\omega^{3}=0$. Following [13], let $\Sigma \subset G\left(2, V_{6}\right)$ be the surface defined as follows:

$$
\Sigma=\left\{[l] \in G\left(2, V_{6}\right):\left.\omega\right|_{V_{l}}=0 \text { for all } \omega \in W_{6}\right\},
$$

where we denote by $V_{l} \subset V_{6}$ the rank-2 vector subspace corresponding to $[l]$ ( $l$ will denote the corresponding projective line in $\mathbb{P}\left(V_{6}\right)$ ). Being defined (for general $X$ as above) as the complete intersection of six linear Plücker forms on $G(2,6), \Sigma$ is a smooth degree-14 K3 surface.

Our goal in this section is to prove the following result.

Theorem 6.2. The intermediate Jacobian fibration $\mathcal{J}$ of $X$ is birational to the O'Grady moduli space $\mathcal{M}_{2,0,4}(\Sigma)$ parameterizing rank-2 semi-stable sheaves on $\Sigma$ with $c_{1}=0$ and $c_{2}=4$.

Corollary 6.3. The compactified Jacobian fibration $\overline{\mathcal{J}}$ of Theorem 5.7 is a deformation of O'Grady's 10-dimensional variety.

Proof. Indeed, Theorem 5.7 is valid when $X$ is a general Pfaffian cubic 4-fold, due to the results of $\S 3.2$ which guarantee that the assumptions needed to make the local construction of $\S 4$ work are satisfied in the general Pfaffian case. Hence we can rephrase Theorem 6.2 saying that our family $\left(\overline{\mathcal{J}}_{X}\right)_{[X] \in W \subset \mathcal{H} y p_{4,3}}$ of hyper-Kähler compactified Jacobian fibrations $\overline{\mathcal{J}}_{X}$ parameterized by an open set $W$ of the space of all cubic 4 -folds has a smooth member which is birational to O'Grady's 10-dimensional variety OG10, which is also a smooth hyper-Kähler manifold. We then apply [33] to conclude that the varieties $\overline{\mathcal{J}}_{X}$ are deformation equivalent to OG10.

We will heavily use the results of [37] (based on [44]) and their generalization in [41]. The proof of Theorem 6.2 will be completed in $\S 6.4$. As the intermediate Jacobian

$\left({ }^{2}\right)$ In fact, the recent paper [39], written with Kollár after this paper was accepted, shows how to use the degeneration to the chordal cubic to give an alternative proof of Theorem 6.2. 
fibration is a fibration over $\mathbb{P}\left(W_{6}^{*}\right)$ into intermediate Jacobians of cubic 3-folds $Y_{3}=H \cap X$, which come equiped with a Pfaffian representation induced from the one of $X$, we will devote $\S 6.2$ and $\S 6.3$ to the Pfaffian 3-fold geometry, which is the subject of the papers [37] and [41]. The cubic 3-fold $Y_{3}$ is represented as the Pfaffian cubic in $\mathbb{P}\left(W_{5}\right)$ for some 5-dimensional vector space $W_{5} \subset \bigwedge^{2} V_{6}^{*}$. Associated with this data, we get the index-1, degree-14 Fano 3-fold $\mathbf{V}_{14} \subset G\left(2, V_{6}\right)$ defined as

$$
\mathbf{V}_{14}=\left\{[l] \in G\left(2, V_{6}\right):\left.\omega\right|_{V_{l}}=0 \text { for all } \omega \in W_{5}\right\} .
$$

Notice that by construction these Fano 3 -fold all contain the K3 surface $\Sigma$ defined above. The strategy of the proof is the following: First one notices that the relative intermediate Jacobian $\mathcal{J}_{U}$ is birational to a relative moduli space of vector bundles $\mathcal{M}$ on the hyperplane sections of $X$ (see beginning of $\S 6.2$ ). Then, with a vector bundle $E_{Y_{3}} \in \mathcal{M}$ on a cubic 3 -fold $Y_{3}$, one would like to associate a vector bundle $E_{\mathbf{V}_{14}}$ on the corresponding degree-14 Fano 3-fold $\mathbf{V}_{14}$ in order to then restrict it to the K3 surface $\Sigma$. However, to define this assignment $E_{Y_{3}} \mapsto E_{\mathbf{V}_{14}}$ we need some more data than $E_{Y_{3}}$ alone, so we end up first getting a map from a variety dominating $\mathcal{J}_{U}$ (map defined in (6.9)), and then showing that this map factors through $\mathcal{J}_{U}$ (Proposition 6.15).

The bundles in question on $Y_{3}$ and on $\mathbf{V}_{14}$ will be obtained via elementary transformations from two natural rank-2 vector bundles on $Y_{3}$ and on $\mathbf{V}_{14}$, which we now define. The variety $\mathbf{V}_{14}$ comes equipped with the tautological rank-2 dual vector bundle, that we will denote by $\mathcal{E}_{14}$. The Pfaffian cubic $Y_{3}$ comes equipped with the natural rank-2 vector bundle $\mathcal{E}_{3}$ with fiber $(\operatorname{Ker} \omega)^{*}$ over a 2 -form $\omega \in \mathbb{P}\left(\bigwedge^{2} V_{6}^{*}\right)$ of rank 4 . As $V_{6}^{*}=H^{0}\left(Y_{3}, \mathcal{E}_{3}\right)=H^{0}\left(\mathbf{V}_{14}, \mathcal{E}_{14}\right)$, we have natural maps

$$
\phi: \mathbb{P}\left(\mathcal{E}_{14}\right) \longrightarrow \mathbb{P}\left(V_{6}\right) \quad \text { and } \quad \psi: \mathbb{P}\left(\mathcal{E}_{3}\right) \longrightarrow \mathbb{P}\left(V_{6}\right),
$$

which are easily seen to have the same image $Q \subset \mathbb{P}\left(V_{6}\right)$. According to [41], $Q$ is a quartic hypersurface which is singular along a curve, and $\phi$ and $\psi$ are two small resolutions of $Q$. In particular, $\mathbb{P}\left(\mathcal{E}_{14}\right)$ and $\mathbb{P}\left(\mathcal{E}_{3}\right)$ are birational, and even related by a flop $\theta=\psi^{-1} \circ \phi$.

We will need the following lemma: Denote by $\Delta_{14}$ (resp. $\Delta_{3}$ ) the general fiber of the projective bundle $\pi_{\mathbf{V}_{14}}: \mathbb{P}\left(\mathcal{E}_{14}\right) \rightarrow \mathbf{V}_{14}$ (resp. $\left.\pi_{Y_{3}}: \mathbb{P}\left(\mathcal{E}_{3}\right) \rightarrow Y_{3}\right)$. Via the birational map $\theta$, these curves (which do not meet the indeterminacy locus of $\theta$ or $\theta^{-1}$ ) can be seen as curves either in $\mathbb{P}\left(\mathcal{E}_{14}\right)$ or in $\mathbb{P}\left(\mathcal{E}_{3}\right)$, and we will denote by $\operatorname{deg}_{\mathbf{V}_{14}}(\cdot)\left(\operatorname{resp} . \operatorname{deg}_{Y_{3}}(\cdot)\right)$ the degree of their projection in $\mathbf{V}_{14}$ (resp. $Y_{3}$ ) with respect to the canonical polarizations. We will also denote by $\operatorname{deg}_{Q}(\cdot)$ the degree of their projections in $Q \subset \mathbb{P}^{5}$ via $\phi($ resp. $\psi$ ).

Lemma 6.4. One has $\operatorname{deg}_{Q} \Delta_{3}=\operatorname{deg}_{Q} \Delta_{14}=1$ and

$$
\operatorname{deg}_{\mathbf{V}_{14}} \Delta_{14}=0, \quad \operatorname{deg}_{Y_{3}} \Delta_{14}=4, \quad \operatorname{deg}_{Y_{3}} \Delta_{3}=0, \quad \operatorname{deg}_{\mathbf{V}_{14}} \Delta_{3}=4 .
$$


Proof. Let $\omega \in Y_{3}$ be a general point. The fiber $\Delta_{3}$ over $\omega$ is thus by definition the projective line $L_{\omega}=\mathbb{P}(\operatorname{Ker} \omega)$. Its image $C_{\omega}$ in $\mathbf{V}_{14}$ is the set of points [l] in $\mathbf{V}_{14}$ such that the corresponding projective line $l$ meets $L_{\omega}$. As $L_{\omega}=\mathbb{P}(\operatorname{Ker} \omega)$, one has $\left.\omega\right|_{V_{l}}=0$ for any line $l$ meeting $L_{\omega}$. Thus $C_{\omega}$ is the vanishing locus of the 4 -dimensional space $W_{5} /\langle\omega\rangle$ of Plücker equations on the set of lines in $V_{6}$ meeting $L_{\omega}$. It is easily proved that this locus has degree 4 and this proves the last statement. Next let $[l] \in \mathbf{V}_{14}$ be a general point. Let $C_{l}$ be the image in $Y_{3}$ of the fiber $\Delta_{14}$ over [l]. Then $C_{l}$ is the set of $\alpha \in W_{5}$ whose kernel intersects $V_{l}$ non-trivially, and the map $l \mapsto C_{l}$ associates with $x \in l$ the unique form $\omega \in \mathbb{P}\left(W_{5}\right)$ such that $x \in \mathbb{P}(\operatorname{Ker} \omega)$. As all forms in $W_{5}$ vanish on $l$, the natural morphism

$$
V_{6} \otimes \mathcal{O}_{l}(-1) \longrightarrow W_{5}^{*} \otimes \mathcal{O}_{l}
$$

of vector bundles over $l=\mathbb{P}^{1}$ factors through $\left(V_{6} / V_{l}\right) \otimes \mathcal{O}_{l}(-1)$, and its cokernel has thus degree 4 , which proves the second statement in (6.4). The other statements are immediate.

\subsection{The cubic 3 -fold side.}

We know by [44], [37] that the moduli space of stable vector bundles of rank 2 on $Y_{3}$ with $c_{1}=0$ and $\operatorname{deg}_{Y_{3}} c_{2}=2$ is birationally isomorphic to $J\left(Y_{3}\right)$ via the Abel-Jacobi map. Let $E$ be such a vector bundle.

LEMma 6.5. If $E$ is general, then the following statements hold:

(i) $\operatorname{dim} H^{0}\left(Y_{3}, E^{*} \otimes \mathcal{E}_{3}\right)=4$.

(ii) Furthermore, if $\left(Y_{3}, E\right)$ is general, for a general section $\sigma \in H^{0}\left(Y_{3}, E^{*} \otimes \mathcal{E}_{3}\right)$, we have an exact sequence

$$
0 \longrightarrow E \stackrel{\sigma}{\longrightarrow} \mathcal{E}_{3} \longrightarrow \mathcal{O}_{S}(C) \longrightarrow 0
$$

where $S \in\left|\mathcal{O}_{Y_{3}}(2)\right|$ is a smooth $\mathrm{K} 3$ surface and $C \subset S$ is a degree-9, genus-5 curve.

Proof. (i) Note that $E \cong E^{*}$, so (i) is equivalent to $\operatorname{dim} H^{0}\left(Y_{3}, E \otimes \mathcal{E}_{3}\right)=4$. We now specialize to the case where $E$ is not locally free, namely $E=\mathcal{I}_{l_{1}} \oplus \mathcal{I}_{l_{2}}$, where $l_{1}$ and $l_{2}$ are two general lines in $Y_{3}$. Then $h^{0}\left(Y_{3}, \mathcal{E}_{3} \otimes \mathcal{I}_{l_{i}}\right)=2$ for $i=1,2$ and $H^{p}\left(Y_{3}, \mathcal{E}_{3} \otimes \mathcal{I}_{l_{i}}\right)=0$ for $i=1,2$ and $p>0$, which implies the result for general $E$ by standard deformation arguments.

(ii) As $E$ is stable, for any $0 \neq \sigma \in H^{0}\left(Y_{3}, E^{*} \otimes \mathcal{E}_{3}\right)$, the generic rank of $\sigma: E \rightarrow \mathcal{E}_{3}$ must be 2 , so that we have the exact sequence (6.3). We claim that the surface $S$ defined by the vanishing of the determinant of $\sigma$ is smooth so that, in particular, the rank of $\sigma$ is 1 along $S$, and Coker $\sigma$ is a line bundle on $S$. We clearly have $S \in\left|\mathcal{O}_{Y_{3}}(2)\right|$ so that, assuming the claim, $S$ is a smooth K3 surface. Finally, the exact sequence (6.3) and 
the fact that $\operatorname{deg}_{Y_{3}} c_{2}(E)=2$ and $\operatorname{deg}_{Y_{3}} c_{2}\left(\mathcal{E}_{3}\right)=5$ immediately imply that the degree of $C$ is 9. To conclude, we observe that $H^{0}\left(Y_{3}, E\right)=0, H^{1}\left(Y_{3}, E\right)=0$ and the exact sequence (6.3) give $H^{0}\left(S, \mathcal{O}_{S}(C)\right)=6$, that is, $g(C)=5$.

We now prove the claim. As the smoothness of the degeneracy surface $S$ is an open property (on the moduli space of 4 -tuples $\left(Y_{3}, E_{3}, E, \sigma\right)$ with $\operatorname{dim} \operatorname{Hom}\left(E, E_{3}\right)=4$, which is known to be irreducible by (i) and [44], [37]), it suffices to show that the conclusion holds for at least one 4-tuple satisfying the property that $\operatorname{dim} \operatorname{Hom}\left(E, E_{3}\right)=4$. It turns out that the result is true generically even in the degenerate case where $E=\mathcal{I}_{l_{1}} \oplus \mathcal{I}_{l_{2}}$. This is proved by working more generally on the universal Pfaffian cubic $Y_{P f}$ in $\mathbb{P}^{14}$, of which $Y_{3}$ is a generic linear section. One easily checks that given two general lines $l_{1}$ and $l_{2}$ contained in $Y_{P f}$, and two sections $\sigma$ and $\tau$ of $\mathcal{E}_{P f} \otimes \mathcal{I}_{l_{1}}$ and $\mathcal{E}_{P f} \otimes \mathcal{I}_{l_{2}}$, respectively, the quadratic equation $q=\sigma \wedge \tau \in H^{0}\left(Y_{P f}, \operatorname{det} \mathcal{E}_{P f}\right)=H^{0}\left(Y_{P f}, \mathcal{O}_{Y_{P f}}(2)\right)$ is of the form $\left.\omega \mapsto \omega^{2}\right|_{W_{4}}$ for some 4-dimensional subspace $W_{4} \subset V_{6}$, and hence defines a rank-6 quadric $Q$ on $\mathbb{P}^{14}$. Comparing the differentials of the defining equations for $Y_{P f}$ and $Q$, one then concludes that $Q \cap Y_{P f}$ is smooth away from the set of $\omega$ 's whose kernel is contained in the codimension-2 linear subspace $W_{4} \subset W_{6}$, which has codimension 4 in $Y_{P f}$. The generic linear section $Y_{3} \subset Y_{P f}$ thus intersects $Q \cap Y_{P f}$ along a smooth surface.

Note that if we restrict (6.3) to $S$, and then to $C$, we conclude, using $\left.\mathcal{O}_{S}(C)\right|_{C}=K_{C}$, that there is a surjective morphism

$$
\left.\mathcal{E}_{3}\right|_{C} \longrightarrow K_{C}
$$

and hence a section

$$
\phi_{\sigma}: C \longrightarrow \mathbb{P}\left(\left.\mathcal{E}_{3}\right|_{C}\right)
$$

such that $\phi_{\sigma}^{*} \mathcal{O}_{\mathbb{P}\left(\mathcal{E}_{3}\right)}(1)=K_{C}$. This morphism induces a surjection

$$
H^{0}\left(\mathbb{P}\left(\mathcal{E}_{3}\right), \mathcal{O}_{\mathbb{P}\left(\mathcal{E}_{3}\right)}(1)\right) \longrightarrow H^{0}\left(C, K_{C}\right),
$$

since both maps $H^{0}\left(Y_{3}, \mathcal{E}_{3}\right) \rightarrow H^{0}\left(S, \mathcal{O}_{S}(C)\right)$ and $H^{0}\left(S, \mathcal{O}_{S}(C)\right) \rightarrow H^{0}\left(C, K_{C}\right)$ are surjective. As $H^{0}\left(Q, \mathcal{O}_{Q}(1)\right)=H^{0}\left(\mathbb{P}\left(\mathcal{E}_{3}\right), \mathcal{O}_{\mathbb{P}}\left(\mathcal{E}_{3}\right)(1)\right)$, we get as well a surjective map

$$
H^{0}\left(Q, \mathcal{O}_{Q}(1)\right) \longrightarrow H^{0}\left(C, K_{C}\right) .
$$

Thus the image of $C$ in $Q \subset \mathbb{P}\left(V_{6}\right)$ via $\psi \circ \phi_{\sigma}$ is a linearly normal canonical genus-5 curve.

The following lemma shows conversely how to recover the bundle from the curve $C \subset \mathbb{P}\left(\mathcal{E}_{3}\right)$.

Lemma 6.6. Let $C \subset \mathbb{P}\left(\mathcal{E}_{3}\right)$ be a general genus-5 curve such that the image $C^{\prime}$ of $C$ in $Y_{3}$ has degree 9, the morphism $C \rightarrow C^{\prime}$ is an isomorphism, and the image of $C$ in 
$Q \subset \mathbb{P}^{5}$ via $\psi \circ \phi_{\sigma}$ is a linearly normal canonical curve. Then, there exist a unique stable vector bundle $E$ on $Y_{3}$ with $c_{1}=0$ and $\operatorname{deg}_{Y_{3}} c_{2}=2$, and a unique $\sigma \in H^{0}\left(Y_{3}, E^{*} \otimes \mathcal{E}_{3}\right)$ determining the line bundle $\mathcal{O}_{S}(C)$ as in Lemma 6.5.

Here "general" means that there is a component of the Hilbert scheme of such curves on which the conclusion holds generically. In fact, this component is the one containing the curves $\phi_{\sigma}(C)$ appearing in Lemma 6.5 and its proof.

Proof. The curve $C^{\prime} \subset Y_{3}$ has degree 9 and genus 5. Assuming the Abel-Jacobi class of $C^{\prime}$ is general in $J\left(Y_{3}\right)$, then (1) $C^{\prime}$ is not contained in a hyperplane section of $Y_{3}$ and (2) $C^{\prime}$ is contained in a unique surface $S \subset Y_{3}$, where $S$ is a member of $\left|\mathcal{O}_{Y_{3}}(2)\right|$. Indeed, curves contained in a hyperplane section of $Y_{3}$ cannot have a general Abel-Jacobi class, by Lemma 6.7 below. This proves the first statement. We have $h^{0}\left(Y_{3}, \mathcal{O}_{Y_{3}}(2)\right)=15$ and $h^{0}\left(C^{\prime},\left.\mathcal{O}_{Y_{3}}(2)\right|_{C^{\prime}}\right)=14$, and thus $C^{\prime}$ is contained in at least one quadric section of $X$. If $C^{\prime}$ is contained in two surfaces $S$ and $S^{\prime}$ as above, then as $S$ and $S^{\prime}$ have no common component by the first statement, $C^{\prime}$ is a component of the complete intersection $S \cap S^{\prime}$ which has degree 12. Thus $C^{\prime}$ is residual to a degree-3 curve, and its Abel-Jacobi point is, up to a sign and a constant, the Abel-Jacobi point of a degree-3 curve which again by Lemma 6.7 cannot be general in $J\left(Y_{3}\right)$. This proves the second statement. Note that, according to Lemma 6.5 (ii), the surface $S$ is smooth for general $C$ in the considered component of the Hilbert scheme of $\mathbb{P}\left(\mathcal{E}_{3}\right)$. We now observe that the restriction map $H^{0}\left(S,\left.\mathcal{E}_{3}^{*}\right|_{S}\left(C^{\prime}\right)\right) \rightarrow H^{0}\left(C^{\prime},\left.\mathcal{E}_{3}^{*}\right|_{C^{\prime}}\left(K_{C^{\prime}}\right)\right)$ is surjective. This indeed follows from the fact that $H^{1}\left(S,\left.\mathcal{E}_{3}^{*}\right|_{S}\right)=0$ (see [41]). Let $\sigma^{\prime} \in H^{0}\left(S,\left.\mathcal{E}_{3}^{*}\right|_{S}\left(C^{\prime}\right)\right.$ ) be a lift of the natural section $\sigma \in H^{0}\left(C^{\prime},\left.\mathcal{E}_{3}^{*}\right|_{C^{\prime}}\left(K_{C^{\prime}}\right)\right)$ giving the embedding of $C^{\prime}$ in $\mathbb{P}\left(\mathcal{E}_{3}\right)$ with image $C$. We have $\operatorname{dim} H^{0}\left(Y_{3}, \mathcal{E}_{3}\right)=6=\operatorname{dim} H^{0}\left(S, \mathcal{O}_{S}(C)\right)$ and thus the property that the map

$$
\sigma^{\prime}: H^{0}\left(Y_{3}, \mathcal{E}_{3}\right) \longrightarrow H^{0}\left(S, \mathcal{O}_{S}(C)\right)
$$

is an isomorphism is an open property. Furthermore, the line bundle $\mathcal{O}_{S}(C)$ is generically globally generated, and we thus conclude that for generic $C$ we get a surjective morphism

$$
\sigma^{\prime}:\left.\mathcal{E}_{3}\right|_{S} \longrightarrow \mathcal{O}_{S}\left(C^{\prime}\right)
$$

and hence as well a surjective morphism

$$
\mathcal{E}_{3} \longrightarrow \mathcal{O}_{S}\left(C^{\prime}\right)
$$

of sheaves on $Y_{3}$. Its kernel provides the desired bundle $E$. The fact that $E$ is stable follows from $H^{0}\left(Y_{3}, E\right)=0$, which is a consequence of the surjectivity, hence injectivity, of the map $H^{0}\left(Y_{3}, \mathcal{E}_{3}\right) \rightarrow H^{0}\left(S, \mathcal{O}_{S}(C)\right)$, which implies that $H^{0}\left(Y_{3}, E\right)=0$. 
We used above the following lemma.

LEMma 6.7. Let $M$ be a smooth variety and $Z \subset M \times Y_{3}$ be a codimension-2 subvariety. Assume that, for general $m \in M$, the curve $Z_{m} \subset Y_{3}$ is contained in a hyperplane section of $Y_{3}$. Then the Abel-Jacobi map $\Phi_{Z}: M \rightarrow J\left(Y_{3}\right)$ is not dominating.

Proof. This immediately follows from the fact that the transpose of the differential of the Abel-Jacobi map

$$
d \Phi_{Z}^{*}: \Omega_{J\left(Y_{3}\right), 0} \longrightarrow \Omega_{M, m}
$$

factors through the restriction map

$$
H^{0}\left(Y_{3}, \mathcal{O}_{Y_{3}}(1)\right) \longrightarrow H^{0}\left(Z_{m}, \mathcal{O}_{Z_{m}}(1)\right),
$$

where one uses the natural identification (see $§ 1.2$ )

$$
\Omega_{J\left(Y_{3}\right), 0} \cong H^{0}\left(Y_{3}, \mathcal{O}_{Y_{3}}(1)\right) .
$$

Our assumptions thus say that $d \Phi_{Z}^{*}$ is not injective, and hence $\Phi_{Z}$ is nowhere a submersion on $M$.

Lemmas 6.5 and 6.6 together show that a component of the family of genus- 5 curves in $\mathbb{P}\left(\mathcal{E}_{3}\right)$, of $Y_{3}$-degree 9 and $Q$-degree 8 , is birationally a $\mathbb{P}^{5}$-bundle over a $\mathbb{P}^{3}$-bundle over a Zariski open set of $J\left(Y_{3}\right)$, which is itself birational to a moduli space of rank-2 vector bundles on $Y_{3}$.

Remark 6.8. We believe that the $\mathbb{P}^{3}$-bundle is not Zariski locally trivial, that is, is not the projectivization of a vector bundle over any Zariski open set of $J\left(Y_{3}\right)$.

\subsection{The $\mathrm{V}_{14}$ side.}

Recall that $\mathbf{V}_{14} \subset G(2,6)$ denotes a smooth 3-dimensional linear section of $G(2,6)$. We are going to study degree-13, genus-5 curves $C \subset \mathbf{V}_{14}$ such that $h^{0}\left(\left.\mathcal{E}_{14}\right|_{C}\right)=6$. By the Riemann-Roch theorem, there is a non-zero morphism

$$
\sigma_{C}:\left.\mathcal{E}_{14}\right|_{C} \longrightarrow K_{C}
$$

When the morphism is surjective, it provides a section $\phi_{\sigma}: C \rightarrow \mathbb{P}\left(\mathcal{E}_{14}\right)$ such that

$$
\phi_{\sigma}^{*}\left(\mathcal{O}_{\mathbb{P}\left(\mathcal{E}_{14}\right)}(1)\right)=K_{C} .
$$

Counting dimensions from the viewpoint of genus-5 curves equipped with a semi-stable rank-2 vector bundle $\mathcal{E}$ of degree 13 with $h^{0}(C, \mathcal{E})=6$, we see that the general such triple $\left(C, \mathcal{E}, \sigma_{C}\right)$ corresponds to a morphism $\sigma_{C}$ which is surjective, which we will assume from now on.

We have the following lemma. 
Lemma 6.9. (i) Such curves $C \subset \mathbf{V}_{14}$ exist for a general smooth $\mathbf{V}_{14} \subset G(2,6)$.

(ii) Let $\mathcal{L}$ be the Plücker line bundle on $G(2,6)$. Then $\operatorname{dim} H^{0}\left(C,\left.\mathcal{L}\right|_{C}\right)=9$ and the restriction map $H^{0}\left(\mathbf{V}_{14}, \mathcal{L}\right) \rightarrow H^{0}\left(C,\left.\mathcal{L}\right|_{C}\right)$ is surjective. Hence $C$ is contained in exactly one $\mathrm{K} 3$ surface $S \in|\mathcal{L}|$.

(iii) The surface $S$ is smooth.

Proof. Note that $\mathbf{V}_{14}$ contains a line $\Delta$. Let now $S_{0} \subset \mathbf{V}_{14}$ be a K3 surface hyperplane section of $\mathbf{V}_{14}$ containing $\Delta$ and having as only singularity a node $x_{0}$ which is not on $\Delta$. Let $\widetilde{S}_{0}$ be the desingularization of $S_{0}$ by blowing-up $x_{0}$. Then $\widetilde{S}_{0}$ contains in its Picard lattice the subgroup generated by the classes $\mathcal{L}_{S}, \Delta$, e, where $e$ is the class of the exceptional curve. The intersection numbers are

$$
\mathcal{L}_{S}^{2}=14, \quad \mathcal{L}_{S} \cdot \Delta=1, \quad \mathcal{L}_{S} \cdot e=0, \quad e^{2}=\Delta^{2}=-2, \quad e \cdot \Delta=0
$$

It thus follows that the curves $\widetilde{C}$ in $\left|\mathcal{L}_{S}-\Delta-e\right|$ have genus 5 and Plücker degree 13 . One easily checks that the general such curve $\widetilde{C}$ satisfies $h^{0}\left(\widetilde{C}, n^{*} \mathcal{E}_{14}\right)=6$, where $n: \widetilde{C} \rightarrow S_{0} \subset$ $G(2,6)$ is the natural map. We now deform the surface $S_{0}$ to a smooth surface $S_{t}$ in $\mathbf{V}_{14}$ on which the class $e+\Delta$ remains algebraic. Then the Picard lattice of the general such smoothing is generated by $\mathcal{L}_{S_{t}}$ and $e+\Delta$, and the class $e+\Delta$ is not effective anymore on $S_{t}$. Hence we also have $H^{1}\left(S_{t}, \mathcal{O}_{S_{t}}(e+\Delta)\right)=0$. It thus follows that the curves $C$ in $\left|\mathcal{L}_{S_{t}}(-e-\Delta)\right|$ have the property that the restriction map

$$
H^{0}\left(S_{t}, \mathcal{L}_{S_{t}}\right) \longrightarrow H^{0}\left(C,\left.\mathcal{L}\right|_{C}\right)
$$

is an isomorphism. This proves the three statements, except for smoothness of the general curves $C \subset S_{t}$ which follows from the fact that the line bundle $\mathcal{L}_{S_{t}}(-e-\Delta)$ is nef. (This is also true for the curves $\widetilde{C}$ on the surface $\widetilde{S}_{0}$, but as it is not embedded in $\mathbf{V}_{14}$, the resulting curves $n(\widetilde{C})$ are nodal.)

Assuming that the curve $C \subset \mathbf{V}_{14}$ is general and thus satisfies the properties above, we now compute that $\chi\left(S,\left.\mathcal{E}_{14}^{*}\right|_{S}(C)\right)=1$ and thus either

$$
H^{0}\left(S,\left.\mathcal{E}_{14}^{*}\right|_{S}(C)\right) \neq 0 \quad \text { or } \quad H^{0}\left(S,\left.\mathcal{E}_{14}\right|_{S}(-C)\right) \neq 0 .
$$

As we have $H^{0}(S, \mathcal{L}(-C))=0$, and $\mathcal{L}=\operatorname{det} \mathcal{E}_{14}$, the second case is excluded, so that we have a non-zero morphism $\sigma: \mathcal{E}_{14} \rightarrow \mathcal{O}_{S}(C)$ extending the morphism $\sigma_{C}$ of (6.5). We now compute

$$
c_{2}\left(\left.\mathcal{E}_{14}^{*}\right|_{S}(C)\right)=c_{2}\left(\left.\mathcal{E}_{14}^{*}\right|_{S}\right)-\operatorname{deg}_{\mathbf{V}_{14}} C+C^{2}=5-13+8=0 .
$$

It thus follows that either $\sigma$ vanishes nowhere on $S$, or $\sigma$ vanishes along a curve in $S$ which does not meet $C$. The second case can only occur if $\varrho(S) \geqslant 3$ while a dimension 
count shows that the family of surfaces $S$ appearing in this construction has dimension 8 , so that the generically $S$ satisfies $\varrho(S)=2$. Hence $\sigma$ is everywhere surjective and we thus get a rank-2 vector bundle $E$ on $\mathbf{V}_{14}$ with trivial determinant fitting in the exact sequence

$$
0 \longrightarrow E \longrightarrow \mathcal{E}_{14} \longrightarrow \mathcal{O}_{S}(C) \longrightarrow 0
$$

One easily computes that $\operatorname{deg}_{\mathbf{V}_{14}}\left(c_{2}(E)\right)=4$. The following will be useful.

Lemma 6.10. The restriction of $E$ to a smooth hyperplane section $\Sigma \subset \mathbf{V}_{14}$ is a rank-2 vector bundle on $\Sigma$ with trivial determinant and $c_{2}=4$. If $\varrho(\Sigma)=1$, then $\left.E\right|_{\Sigma}$ is stable.

Proof. The first statement is obvious. The stability follows from the vanishing $H^{0}\left(\Sigma,\left.E\right|_{\Sigma}\right)=0$, which is implied by $H^{0}\left(\mathbf{V}_{14}, E\right)=0$ and $H^{1}\left(\mathbf{V}_{14}, E \otimes \mathcal{L}^{-1}\right)=0$, which are both implied by the exact sequence (6.6). One uses the fact that (6.6) induces an isomorphism on the level of global sections to get $H^{0}\left(\mathbf{V}_{14}, E\right)=0$, and the fact that $\left(C-c_{1}(\mathcal{L})\right)^{2}=-4$ on $S$, so that $H^{0}\left(S, \mathcal{L}^{-1}(C)\right)=0$, to get $H^{1}\left(\mathbf{V}_{14}, E \otimes \mathcal{L}^{-1}\right)=0$.

Remark 6.11. Note that the vector bundle $E$ constructed above from the data of the K3 surface $S$ and the line bundle $\mathcal{O}_{S}(C)$ satisfies $\operatorname{dim} \operatorname{Hom}\left(E, \mathcal{E}_{14}\right)=4$, as easily follows from (6.6). It follows that the 13-dimensional family of genus-5, degree-13 curves on $\mathbf{V}_{14}$ corresponds in fact to a 5-dimensional family of vector bundles on $\mathbf{V}_{14}$.

\subsection{Construction of the rational map}

We now make the following construction: Let $\left(Y_{3}, \mathcal{E}_{3}\right)$ be a general cubic 3 -fold with Pfaffian structure, and let $E$ be a general rank-2 stable vector bundle on $Y_{3}$ with $c_{1}(E)=0$ and $\operatorname{deg}_{Y_{3}} c_{2}(E)=2$. By Lemma 6.5, there is an associated 8-dimensional family of genus5 curves $C \subset \mathbb{P}\left(\mathcal{E}_{3}\right)$ satisfying $\operatorname{deg}_{Y_{3}}(C)=9$ and $\operatorname{deg}_{Q}(C)=8$ such that the image $\psi(C) \subset Q$ is a linearly normal canonical curve of genus 5 . We claim that the curve $\psi(C)$ is the complete intersection of three quadrics in $\mathbb{P}^{4}$ : For this, we have to show that $C$ is not trigonal. However, from our construction, we see that $C$ is contained in a general K3 surface $S$ with Picard lattice generated by $h$ and the class $c$ of $C$, with intersection lattice

$$
h^{2}=6, \quad h \cdot c=9, \quad c^{2}=8
$$

The fact that $C$ is not trigonal then follows from [26]. As $\psi(C) \subset Q$ is the complete intersection of three quadrics in a hyperplane section $H \cap Q$ of $Q$, we can write

$$
\left.q\right|_{H}=s_{1} q_{1}+s_{2} q_{2}+s_{3} q_{3},
$$


where $q$ is the defining equation for $Q$, and the $q_{i}$ 's are the defining equations for $\psi(C)$. Here the $s_{i}$ 's are also quadratic polynomials on $H$. It follows that $Q$ contains another set of canonical curves of genus 5 , namely, viewing the expression in the right-hand side of (6.7) as a quadric in the six variables $q_{i}$ and $s_{i}, i=1,2,3$, the plane defined by the $q_{i}$ 's determines one ruling of this quadric (these planes are parameterized by a $\mathbb{P}^{3}$ ) and the planes in the other rulings will correspond to a second $\mathbb{P}^{3}$ of linearly normal degree- 8 genus-5 canonical curves in $Q \cap H$. Concretely, the curve $C_{1} \subset Q \cap H$ defined in $H$ by $q_{1}=q_{2}=s_{3}=0$ is such a curve. The important point for us is that the original curve $C$ is a general member of a linear system $\left|\mathcal{O}_{S}(C)\right|$ on a K3 surface $S \subset \mathbb{P}\left(\mathcal{E}_{3}\right)$, and hence it does not meet the surface $\Sigma_{3} \subset \mathbb{P}\left(\mathcal{E}_{3}\right)$ which is contracted by $\psi$, which means that $\psi(C)$ does not meet the singular curve of $Q$. The residual curve $C_{1}$ constructed above thus moves freely in $Q$ and also avoids the singular locus of $Q$ which is the indeterminacy locus of the rational map $\phi^{-1}$. Thus it lifts to a curve $C^{\prime}=\phi^{-1}\left(C_{1}\right) \subset \mathbb{P}\left(\mathcal{E}_{14}\right)$.

Lemma 6.12. The genus-5 curve $C^{\prime}$ satisfies $\operatorname{deg}_{\mathbf{V}_{14}} C^{\prime}=13$.

Proof. The rank-2 vector space

$$
H^{6}\left(\mathbb{P}\left(\mathcal{E}_{14}\right), \mathbb{Q}\right)_{\text {alg }}=\operatorname{Hdg}^{6}\left(\mathbb{P}\left(\mathcal{E}_{14}\right)\right)=H^{6}\left(\mathbb{P}\left(\mathcal{E}_{3}\right), \mathbb{Q}\right)_{\text {alg }}
$$

of curve classes in either of these two varieties is generated by the classes $\left[\Delta_{3}\right]$ and $\left[\Delta_{14}\right]$. We can thus write in this space

$$
[C]=\alpha\left[\Delta_{3}\right]+\beta\left[\Delta_{14}\right]
$$

Next, as the curve $C_{1}=\phi\left(C^{\prime}\right) \subset Q$ is residual to $\psi(C)$ in the complete intersection in $Q$ of a hyperplane $H$ and two quadrics, we get that $\left[C^{\prime}\right]=4 h_{Q}^{3}-[C]$ in $A^{3}\left(\mathbb{P}\left(\mathcal{E}_{14}\right)\right)$, where $h_{Q}$ is the pull-back to $\mathbb{P}\left(\mathcal{E}_{14}\right)$ of $c_{1}\left(\mathcal{O}_{Q}(1)\right)$. Note that we also have $h_{Q}=c_{1}\left(\mathcal{O}_{\mathbb{P}\left(\mathcal{E}_{14}\right)}(1)\right)$. It thus follows that

$$
\operatorname{deg}_{\mathbf{V}_{14}} C^{\prime}=4 \operatorname{deg}_{\mathbf{V}_{14}} h_{Q}^{3}-\operatorname{deg}_{\mathbf{V}_{14}} C .
$$

As $h_{Q}=c_{1}\left(\mathcal{O}_{\mathbb{P}\left(\mathcal{E}_{14}\right)}(1)\right)$, the standard theory of Chern classes (see [25]) says that

$$
\pi_{\mathbf{V}_{14 *}}\left(h_{Q}^{3}\right)=s_{2}\left(\mathcal{E}_{14}\right)=c_{1}^{2}\left(\mathcal{E}_{14}\right)-c_{2}\left(\mathcal{E}_{14}\right),
$$

and thus $\operatorname{deg}_{\mathbf{V}_{14}}\left(h_{Q}^{3}\right)=14-5=9$. Next we have $\operatorname{deg}_{Y_{3}}(C)=9$ and $\operatorname{deg}_{Q}(C)=8$, which by Lemma 6.4 gives

$$
\alpha+\beta=8 \text { and } 4 \beta=9 .
$$

We thus deduce that $4 \alpha=23$. This finally gives, using (6.8),

$$
\operatorname{deg}_{\mathbf{V}_{14}} C^{\prime}=36-4 \alpha=36-23=13 .
$$


By a dimension count (or by the reversibility of the construction), we observe that for generic $C$ the curve $C^{\prime}$ of genus $5, \mathbf{V}_{14}$-degree 13 and $Q$-degree 8 is generic in $\mathbb{P}\left(\mathcal{E}_{14}\right)$, and we can thus apply the construction of $\S 6.3$ to get from $C^{\prime}$ a stable rank-2 vector bundle $E^{\prime}$ on $\mathbf{V}_{14}$ with trivial determinant and $\operatorname{deg}_{\mathbf{V}_{14}} c_{2}\left(E^{\prime}\right)=4$.

Proof of Theorem 6.2. Let $X$ be a general Pfaffian cubic 4-fold and let $u: \mathcal{Y}_{U} \rightarrow U$ be the universal family of smooth hyperplane sections of $X$. The general fiber of $u$ is thus a general cubic 3-fold with Pfaffian structure. For each point $t \in U$, there is a canonical morphism from the moduli space $\mathcal{M}_{2,0,2}\left(Y_{t}\right)$ of rank-2 vector bundles on $Y_{t}$ with trivial determinant and $\operatorname{deg}_{Y_{t}} c_{2}=2$ to the intermediate Jacobian $J\left(Y_{t}\right)$ which maps $E$ to $\Phi_{Y_{t}}\left(c_{2}(E)-c_{2}\left(\mathcal{E}_{3, t}(-1)\right)\right)$. Here $\mathcal{E}_{3, t}$ denotes the restriction to $Y_{t}$ of the Pfaffian vector bundle $\mathcal{E}$ on $X$. This morphism is birational by [37]. In this way we conclude that the moduli space $\mathcal{M}$ of sheaves on $X$ supported on a hyperplane section and with the same numerical data as $E$ (seen as a sheaf on $X$ ) is birational to $\mathcal{J}_{U}$, where $\mathcal{J}_{U} \rightarrow U$ is the family of intermediate Jacobians.

Remark 6.13. For general $X$, this birational isomorphism does not exist, or rather takes values in a torsor under $\mathcal{J}$.

On the other hand, we also have the universal family

$$
v: \mathcal{V}_{14, U} \rightarrow U
$$

of corresponding linear sections of the Grassmannian. For each $t \in U$ corresponding to a $W_{5, t} \subset W_{6} \subset \bigwedge^{2} V_{6}^{*}$, the fiber $\mathcal{V}_{14, t}$ is the complete intersection of $G\left(2, V_{6}\right)$ with five Plücker hypersurfaces defined by $W_{5, t}$. We thus have a natural inclusion $\Sigma \subset \mathcal{V}_{14, t}$ as a Plücker hypersurface, since by definition $\Sigma \subset G\left(2, V_{6}\right)$ is the vanishing locus in $G\left(2, V_{6}\right)$ of the six Plücker equations defined by $W_{6}$.

The construction described above done in family over $U$ now gives us the following: There exists a smooth projective variety $W$ which admits a morphism $g: W \rightarrow \mathcal{M} \cong$ birat $\mathcal{J}_{U}$ with rationally connected fibers and a rational map

$$
f: W \rightarrow \mathcal{M}_{2,0,4}(\Sigma)
$$

The general point of $W$ parameterizes a general rank-2 vector bundle $E$ with $c_{1}=0$ and $\operatorname{deg}_{Y_{t}} c_{2}=2$ on a fiber $Y_{t}$ of $\mathcal{Y}_{U}$, the choice of a general non-zero morphism $\sigma: E \rightarrow \mathcal{E}_{3, t}$ defined up to a coefficient, a general member $C$ of the linear system $\left|\mathcal{O}_{S}(C)\right|$, where $\mathcal{O}_{S}(C)$ is defined by the exact sequence (6.3), and a general $(2,2,2,1)$ complete intersection curve $C^{\prime}$ contained in $Q_{t}$, residual in $Q_{t}$ to the $(2,2,2,1)$ complete intersection curve $\psi_{t}(C) \subset Q_{t}$ (we will see in fact $C^{\prime}$ as living in $\mathbb{P}\left(\mathcal{E}_{14, t}\right)$ ). Thus, the general fiber of the map $g$ has 
dimension 10 , and $W$ has dimension $20=10+10$. The rational map $f$ then associates with these data the vector bundle $\left.E^{\prime}\right|_{\Sigma}$, which is stable by Lemma 6.10 , where the vector bundle $E^{\prime}=E_{S^{\prime}, C^{\prime}}$ on $\mathcal{V}_{14, t}$ is associated as in $\S 6.3$ with the curve $C^{\prime}$. Here $S^{\prime}$ is the generically unique Plücker hyperplane section of $\mathcal{V}_{14, t}$ containing $C^{\prime}$.

Remark 6.14. One easily checks that $E^{\prime}$ does not depend on the choice of $C$ or the residual curve $C^{\prime}$. The only reason to introduce these curves was the fact that they do not meet the singular locus of $Q_{t}$, which is not true for the associated K3 surfaces where they lie. It will also appear below that $E^{\prime}$ neither depends on the choice of $\sigma$.

The proof of Theorem 6.2 will be completed using the following.

Proposition 6.15. The rational map $f$ factors through $\mathcal{J}_{U}$ and induces a birational isomorphism

$$
g: \mathcal{J}_{U} \rightarrow-\mathcal{M}_{2,0,4}(\Sigma)
$$

Proof. We know by Theorem 5.7 that $\mathcal{J}_{U}$ is not uniruled (this is indeed implied by the fact that a smooth projective completion of $\mathcal{J}_{U}$ admits a generically non-degenerate holomorphic 2 -form). It follows that $\mathcal{J}_{U}$ is birational to the basis of the maximal rationally connected fibration of $W$. We now have the following lemma.

Lemma 6.16. (i) The rational map $f$ is dominating.

(ii) The general fiber of $f$ is rationally connected.

Proof. (i) The variety $W$ has two holomorphic (in fact algebraic) 2-forms, namely the pull-back $\tilde{\sigma}_{\mathcal{J}}$ to $W$ of the holomorphic 2-form $\sigma_{\mathcal{J}}$ on $\mathcal{J}_{U}$ constructed in Theorem 5.7, and the form $f^{*} \sigma_{\mathcal{M}_{2,0,4}}$. We claim that, for some $\lambda \neq 0$,

$$
\tilde{\sigma}_{\mathcal{J}}=\lambda f^{*} \sigma_{\mathcal{M}_{2,0,4}}
$$

This equation immediately implies the surjectivity of $f$, since the generic rank of $\tilde{\sigma}_{\mathcal{J}}$ is equal to $\operatorname{dim} \mathcal{J}_{U}=10$ and the rank of $f^{*} \sigma_{\mathcal{M}_{2,0,4}}$ is not greater than the rank of $f$, so the equality (6.10) implies that the generic rank of $f$ is $10=\operatorname{dim} \mathcal{M}_{2,0,4}(\Sigma)$, implying that $f$ is dominant. We prove now the claim: Note that $W$ is a fibration over $\mathcal{J}_{U}$ (or rather a smooth projective compactification $\overline{\mathcal{J}}$ of $\mathcal{J}_{U}$ ) with rationally connected general fiber, hence $H^{2,0}(W)=H^{2,0}(\overline{\mathcal{J}})$ is of dimension 1 by Theorem 5.7 (iii). As $\tilde{\sigma}_{\mathcal{J}} \neq 0$, it thus suffices to prove that $f^{*} \sigma_{\mathcal{M}_{2,0,4}} \neq 0$. This can be proved by a Chow-theoretic argument using Mumford's theorem [48]. Indeed, there is a natural inclusion

$$
\Sigma \times U \subset \mathcal{V}_{14, U},
$$

which is the restriction over $U$ of the natural inclusion

$$
j: \Sigma \times \mathbb{P}^{5} \subset \mathcal{V}_{14}
$$


where $\mathcal{V}_{14}$ is the universal family of 3 -folds $\mathbf{V}_{14}$ containing $\Sigma$. It is immediate to check that $\operatorname{pr}_{1 *} \circ j^{*}: \mathrm{CH}_{1}\left(\mathcal{V}_{14}\right)_{\text {hom }} \rightarrow \mathrm{CH}_{0}(\Sigma)_{\text {hom }}$ (which is just the restriction map $j_{t}^{*}$ on each $\mathrm{CH}_{1}\left(\mathcal{V}_{14, t}\right)_{\text {hom }}$, where $j_{t}$ is the inclusion of $\Sigma$ in $\left.\mathcal{V}_{14, t}\right)$ is an isomorphism. On the other hand, $\mathcal{V}_{14}$ is birationally equivalent to the universal family $\mathcal{Y}$ of hyperplane sections of $X$, which satisfies $\mathrm{CH}_{1}(\mathcal{Y})_{\text {hom }} \cong \mathrm{CH}_{1}(X)_{\text {hom }}$ since $\mathrm{CH}_{0}(X)_{\text {hom }}=0$ and $\mathcal{Y}$ is a projective bundle over $X$. This fibered birational isomorphism induces an isomorphism between the intermediate Jacobian fibrations over $U$ (see [37]), and hence a fiberwise isomorphism

$$
\mathrm{CH}_{1}\left(\mathcal{V}_{14, t}\right)_{\text {hom }} \cong \mathrm{CH}_{1}\left(Y_{t}\right)_{\text {hom }},
$$

since for rationally connected 3-folds $Y$ the Abel-Jacobi map $\mathrm{CH}_{1}(Y)_{\text {hom }} \rightarrow J(Y)$ is an isomorphism (see [14]). This easily implies that

$$
\mathrm{CH}_{1}\left(\mathcal{V}_{14}\right)_{\mathbb{Q}, \text { hom }} \cong \mathrm{CH}_{1}(\mathcal{Y})_{\mathbb{Q}, \text { hom }}
$$

since $\mathrm{CH}_{0}\left(\mathcal{V}_{14, t}\right)_{\text {hom }}=0$ and $\mathrm{CH}_{0}\left(Y_{t}\right)_{\text {hom }}=0$. We now observe that each point $w$ of the fiber $W_{t}$ of the variety $W$ over $U$ parameterizes vector bundles $E_{w}^{\prime}$ (resp. $E_{w}$ ) on the fibers $\mathcal{V}_{14, t}\left(\right.$ resp. $\left.Y_{t}\right)$, and that for each $t \in U$ the two maps

$$
\begin{aligned}
& c_{V}: W_{t} \longrightarrow \mathrm{CH}_{1}\left(\mathcal{V}_{14, t}\right), \quad \text { and } \quad c_{Y}: W \longrightarrow \mathrm{CH}_{1}\left(Y_{t}\right) \text {, } \\
& w \longmapsto c_{2}\left(E_{w}^{\prime}\right), \quad w \longmapsto c_{2}\left(E_{w}\right),
\end{aligned}
$$

coincide, up to sign and a constant via (6.11). With the notation above, this follows from the construction for a given $t$ of the curve $C^{\prime}$ as residual to the curve $C$ in a $(2,2,1)$ complete intersection in $Q_{t}$. Combining these observations, we conclude that the map

$$
\begin{aligned}
\mathrm{CH}_{0}(W)_{\text {hom }} & \longrightarrow \mathrm{CH}_{0}(\Sigma)_{\text {hom }}, \\
w & \longmapsto c_{2}\left(\left.E_{w}^{\prime}\right|_{\Sigma}\right),
\end{aligned}
$$

is surjective, and hence, by Mumford's theorem [48], that the corresponding pull-back of the holomorphic 2-form on $\Sigma$ is non-zero. However, by construction of the holomorphic 2-form on $\mathcal{M}_{2,0,4}(\Sigma)$, this pull-back is nothing but $f^{*} \sigma_{\mathcal{M}_{2,0,4}}$.

(ii) Let $E$ be a general stable rank-2 vector bundle on $\Sigma$ with trivial determinant and $c_{2}=4$. The fiber of $f$ over $E$ essentially consists of vector bundles $E_{S^{\prime}, C^{\prime}, t}$ on 3 -folds $\mathcal{V}_{14, t}$ containing $\Sigma$ such that

$$
\left.E_{S^{\prime}, C^{\prime}, t}\right|_{\Sigma} \cong E \text {. }
$$

More precisely, for each such vector bundle, one can apply the results of $\S 6.3$ : choosing a general section $\tilde{\sigma}$ of $\operatorname{Hom}\left(E_{S^{\prime}, C^{\prime}, t}, \mathcal{E}_{14, t}\right)$, one gets a degeneracy K3 surface $S_{\tilde{\sigma}} \subset \mathcal{V}_{14, t}$ and a line bundle $\mathcal{O}_{S_{\tilde{\sigma}}}(D)$ on $S_{\tilde{\sigma}}$ which is a quotient of $\left.\mathcal{E}_{14, t}\right|_{S_{\tilde{\sigma}}}$, providing a section 
$\phi_{\tilde{\sigma}}: S_{\tilde{\sigma}} \rightarrow \mathbb{P}\left(\mathcal{E}_{14, t}\right)$. For a general curve $D_{0} \in\left|\mathcal{O}_{S_{\tilde{\sigma}}}(D)\right|$, the lifted curve $\phi_{\tilde{\sigma}}\left(D_{0}\right)$ is of genus 5 , $Q$-degree 8 and $\mathbf{V}_{14}$-degree 13 and using the map $\phi: \mathbb{P}\left(\mathcal{E}_{14, t}\right) \rightarrow Q_{t}$, it provides a complete intersection curves of type $(1,2,2,2)$ contained in $Q_{t}$, and the residual curve $D_{0}^{\prime}$ in a $(2,2,1)$ complete intersection of $Q_{t}$ provides a genus-5 curve in $\mathbb{P}\left(\mathcal{E}_{3, t}\right)$ of $Q$-degree 8 and $Y_{3}$-degree 9. Applying Lemma 6.6, we then reconstruct an element of $W$ with image $E$ under $f$. Given the vector bundle $E_{S^{\prime}, C^{\prime}, t}$ on a 3 -fold $\mathcal{V}_{14, t}$, the extra data described above, namely the choices of $\sigma$ and of the curves $D_{0}$ and $D_{0}^{\prime}$, are parameterized by a rationally connected variety, so the proof will be finished once we know that $E_{S^{\prime}, C^{\prime}, t}$ is determined by $E$.

LEMma 6.17. Let $E_{S^{\prime}, C^{\prime}, t}$ be a general rank-2 vector bundle on $\mathcal{V}_{14, t}$ constructed as in $\S 6.3$, and let $E$ be its restriction to $\Sigma$. Then the restriction map

$$
\operatorname{Hom}\left(E_{S^{\prime}, C^{\prime}, t}, \mathcal{E}_{14, t}\right) \longrightarrow \operatorname{Hom}\left(E,\left.\mathcal{E}_{14}\right|_{\Sigma}\right)
$$

is an isomorphism, where $\left.\mathcal{E}_{14}\right|_{\Sigma}$ denotes the Plücker rank-2 vector bundle restricted to $\Sigma$.

Proof. Indeed, the injectivity is obvious and, on the other hand, both sides have dimension 4 . This was already proved in Remark 6.11 for the left-hand side. For the righthand side, we can specialize the general vector bundle $E$ to the case where $E^{*}=\mathcal{I}_{z_{1}} \oplus \mathcal{I}_{z_{2}}$ where $z_{1}$ and $z_{2}$ are two length-2 subschemes of $\Sigma$; then

$$
H^{0}\left(\Sigma,\left.E^{*} \otimes \mathcal{E}_{14}\right|_{\Sigma}\right)=H^{0}\left(\Sigma,\left.\mathcal{E}_{14}\right|_{\Sigma} \otimes \mathcal{I}_{z_{1}}\right) \oplus H^{0}\left(\Sigma,\left.\mathcal{E}_{14}\right|_{\Sigma} \otimes \mathcal{I}_{z_{2}}\right)
$$

has dimension 4, while

$$
H^{i}\left(\Sigma,\left.E^{*} \otimes \mathcal{E}_{14}\right|_{\Sigma}\right)=H^{i}\left(\Sigma,\left.\mathcal{E}_{14}\right|_{\Sigma} \otimes \mathcal{I}_{z_{1}}\right) \oplus H^{i}\left(\Sigma,\left.\mathcal{E}_{14}\right|_{\Sigma} \otimes \mathcal{I}_{z_{2}}\right)=0 \quad \text { for } i>0 .
$$

The conclusion then follows from a deformation argument.

Let now $\sigma \in \operatorname{Hom}\left(E,\left.\mathcal{E}_{14}\right|_{\Sigma}\right)$ be a general section. Then we get a degeneracy curve $D_{\sigma} \in|\mathcal{L}|_{\Sigma} \mid$, where $\mathcal{L}$ is the Plücker line bundle, and an exact sequence

$$
\left.0 \longrightarrow E \longrightarrow \mathcal{E}_{14}\right|_{\Sigma} \longrightarrow \mathcal{O}_{D_{\sigma}}(Z) \longrightarrow 0,
$$

where $Z$ is a divisor of degree 13 on $D_{\sigma}$. This gives a section $\phi_{\sigma}: D_{\sigma} \rightarrow \mathbb{P}\left(\mathcal{E}_{14}\right)$ with image $\widetilde{D}_{\sigma}$. For each vector bundle $E_{S^{\prime}, C^{\prime}, t}$ on some $\mathcal{V}_{14, t} \supset \Sigma$ restricting to $E$ on $\Sigma$, the section $\sigma$ extends to a section $\tilde{\sigma}$ by Lemma 6.17 , and thus there is a K3 surface

$$
\widetilde{S}_{\tilde{\sigma}}:=\phi_{\tilde{\sigma}}\left(S_{\tilde{\sigma}}\right) \subset \mathbb{P}\left(\mathcal{E}_{14}\right),
$$

which is a lift of the degeneracy surface $S_{\tilde{\sigma}} \subset \mathcal{V}_{14, t}$. The surface $\widetilde{S}_{\tilde{\sigma}}$ intersects $\mathbb{P}\left(\left.\mathcal{E}_{14}\right|_{\Sigma}\right)$ along the curve $\widetilde{D}_{\sigma}=\phi_{\sigma}\left(D_{\sigma}\right)$. The surface $S_{\tilde{\sigma}}$ carries a line bundle $\mathcal{O}_{S_{\tilde{\sigma}}}\left(C_{\sigma}\right)$ which restricts to $\mathcal{O}_{D \sigma}(Z)$ on $D_{\sigma}$. Note that the curve $D_{\sigma} \subset S_{\tilde{\sigma}}$ is a member of $|\mathcal{L}|_{S_{\tilde{\sigma}}} \mid$. The uniqueness of $E_{S^{\prime}, C^{\prime}, t}$ then follows from the results of $\S 6.3$ and from the following lemma. 
LEMma 6.18. For a general curve $\widetilde{D}_{\sigma} \subset \mathbb{P}\left(\mathcal{E}_{14}\right)$ as above, there exists a unique surface $\widetilde{S}_{\tilde{\sigma}} \subset \mathbb{P}\left(\mathcal{E}_{14}\right)$ satisfying the conditions above, that is, lifting a $\mathrm{K} 3$ surface in some $V_{14, t}$ containing $\Sigma$ and intersecting $\mathbb{P}\left(\left.\mathcal{E}_{14}\right|_{\Sigma}\right)$ along the curve $\widetilde{D}_{\sigma}$.

Proof. Let $N_{\widetilde{D}_{\sigma} / \mathbb{P}\left(\mathcal{E}_{14}\right)}$ be the normal bundle of $\widetilde{D}_{\sigma}$ in $\mathbb{P}\left(\mathcal{E}_{14}\right)$. There is an exact sequence

$$
\left.0 \rightarrow T_{\mathbb{P}\left(\mathcal{E}_{14}\right) / G(2,6)}\right|_{\widetilde{D}_{\sigma}} \longrightarrow N_{\widetilde{D}_{\sigma} / \mathbb{P}\left(\mathcal{E}_{14}\right)} \longrightarrow N_{D_{\sigma} / G(2,6)}=\left(\left.\mathcal{L}\right|_{D_{\sigma}}\right)^{7} \longrightarrow 0
$$

and each surface $\widetilde{S}_{\tilde{\sigma}}$ extending $\widetilde{D}_{\sigma}$ as above provides an inclusion

$$
N_{D_{\sigma} / S_{\tilde{\sigma}}}=\left.\mathcal{L}\right|_{D_{\sigma}} \subset N_{\widetilde{D}_{\sigma} / \mathbb{P}\left(\mathcal{E}_{14}\right)},
$$

or equivalently a non-zero section of $N_{\widetilde{D}_{\sigma} / \mathbb{P}\left(\mathcal{E}_{14}\right)} \otimes \mathcal{L}^{-1}$. It is not hard to see that this section determines the surface $\widetilde{S}_{\tilde{\sigma}}$, so we only have to prove that, for general $\widetilde{D}_{\sigma}$ as above, one has

$$
h^{0}\left(\widetilde{D}_{\sigma}, N_{\widetilde{D}_{\sigma} / \mathbb{P}\left(\mathcal{E}_{14}\right)} \otimes \mathcal{L}^{-1}\right)=1
$$

In order to prove (6.13), we write the normal bundle sequence twisted by $\mathcal{L}^{-1}$ for $\widetilde{D}_{\sigma} \subset$ $\widetilde{S}_{\tilde{\sigma}} \subset \mathbb{P}\left(\mathcal{E}_{14}\right)$. This gives

$$
\left.0 \longrightarrow \mathcal{O}_{D_{\sigma}} \longrightarrow N_{\widetilde{D}_{\sigma} / \mathbb{P}\left(\mathcal{E}_{14}\right)} \otimes \mathcal{L}^{-1} \longrightarrow N_{\widetilde{S}_{\tilde{\sigma}} / \mathbb{P}\left(\mathcal{E}_{14}\right)}\right|_{\widetilde{D}_{\sigma}} \otimes \mathcal{L}^{-1} \longrightarrow 0
$$

and (6.13) will follow from $h^{0}\left(\widetilde{D}_{\sigma},\left.N_{\widetilde{S}_{\tilde{\sigma}} / \mathbb{P}\left(\mathcal{E}_{14}\right)}\right|_{\widetilde{D}_{\sigma}} \otimes \mathcal{L}^{-1}\right)=0$, which itself will be a consequence of

$$
h^{0}\left(\widetilde{S}_{\tilde{\sigma}}, N_{\widetilde{S}_{\tilde{\sigma} / \mathbb{P}\left(\mathcal{E}_{14}\right)}} \otimes \mathcal{L}^{-1}\right)=0 \quad \text { and } \quad h^{1}\left(\widetilde{S}_{\tilde{\sigma}}, N_{\widetilde{S}_{\tilde{\sigma}} / \mathbb{P}\left(\mathcal{E}_{14}\right)} \otimes \mathcal{L}^{-2}\right)=0
$$

The second vanishing statement is obtained by writing the normal bundle sequence $(6.12)$ for $\widetilde{S}_{\tilde{\sigma}}$ :

$$
\left.0 \longrightarrow T_{\mathbb{P}\left(\mathcal{E}_{14}\right) / G(2,6)}\right|_{\widetilde{S}_{\tilde{\sigma}}} \longrightarrow N_{\widetilde{S}_{\tilde{\sigma}} / \mathbb{P}\left(\mathcal{E}_{14}\right)} \longrightarrow N_{S_{\tilde{\sigma}} / G(2,6)}=\left(\left.\mathcal{L}\right|_{S_{\tilde{\sigma}}}\right)^{6} \longrightarrow 0
$$

where the line bundle $\left.T_{\mathbb{P}\left(\mathcal{E}_{14}\right) / G(2,6)}\right|_{\widetilde{S}_{\tilde{\sigma}}}$ is isomorphic to $\left(\left.\mathcal{L}\right|_{S_{\tilde{\sigma}}}\right)^{-1}\left(2 C_{\sigma}\right)$. One then concludes using

$$
H^{1}\left(S_{\tilde{\sigma}},\left(\left.\mathcal{L}\right|_{S_{\tilde{\sigma}}}\right)^{-1}\right)=0 \quad \text { and } \quad H^{1}\left(S_{\tilde{\sigma}},\left(\left.\mathcal{L}\right|_{S_{\tilde{\sigma}}}\right)^{-3}\left(2 C_{\sigma}\right)\right)=0
$$

which both follow from standard vanishing theorems on the K3 surface $S_{\tilde{\sigma}}$. It remains to prove the first vanishing statement. However, according to $\S 6.3$, the deformation space of $\widetilde{S}_{\tilde{\sigma}}$ in $\mathbb{P}\left(\mathcal{E}_{14}\right)$ is smooth and isomorphic to a $\mathbb{P}^{3}$-bundle over the 10-dimensional moduli 
space of sheaves on $\mathcal{V}_{14}$ that are supported on the fibers $\mathcal{V}_{14, t}$ and that are locally free on $\mathcal{V}_{14, t}$ of rank 2 , with trivial determinant and $\operatorname{deg}_{\mathbf{V}_{14}} c_{2}=4$. By (i) and Lemma 6.17, the restriction $\operatorname{map}\left(E_{t}, \tilde{\sigma}\right) \rightarrow\left(\left.E_{t}\right|_{\Sigma},\left.\tilde{\sigma}\right|_{\Sigma}\right)$ has generically surjective differential, hence also injective differential. It is clear however that sections of $N_{\widetilde{S}_{\tilde{\sigma}} / \mathbb{P}\left(\mathcal{E}_{14}\right)} \otimes \mathcal{L}^{-1}$, seen as sections of $N_{\widetilde{S}_{\tilde{\sigma}} / \mathbb{P}\left(\mathcal{E}_{14}\right)}$ vanishing on $\widetilde{D}_{\sigma}$, belong to the kernel of this differential. Hence they must be trivial.

This concludes the proof of Lemma 6.16.

Lemma 6.16 implies Proposition 6.15 as follows: since $f$ is dominating by (i) and $\mathcal{M}_{2,0,4}(\Sigma)$ is not uniruled, $f$ must factor through the maximal rationally connected fibration of $W$, that is, through $\mathcal{J}_{U}$. The general fiber of the induced rational map $g: \mathcal{J}_{U^{--\rightarrow}} \mathcal{M}_{2,0,4}(\Sigma)$ are then rationally connected by (ii). But, as $\mathcal{J}_{U}$ is not uniruled, the general fiber of $g$ is a point, so $g$ is birational.

The proof of Theorem 6.2 is now finished.

\section{References}

[1] Addington, N. \& Lehn, M., On the symplectic eightfold associated to a Pfaffian cubic fourfold. To appear in J. Reine Angew. Math.

[2] Alexeev, V., Birkenhake, C. \& Hulek, K., Degenerations of Prym varieties. J. Reine Angew. Math., 553 (2002), 73-116.

[3] Allcock, D., The moduli space of cubic threefolds. J. Algebraic Geom., 12 (2003), 201223.

[4] Altman, A. B. \& Kleiman, S. L., Compactifying the Picard scheme. II. Amer. J. Math., 101 (1979), 10-41.

[5] - Compactifying the Picard scheme. Adv. Math., 35 (1980), 50-112.

[6] Amerik, E., A computation of invariants of a rational self-map. Ann. Fac. Sci. Toulouse Math., 18 (2009), 445-457.

[7] Arbarello, E., Saccì, G. \& Ferretti, A., Relative Prym varieties associated to the double cover of an Enriques surface. J. Differential Geom., 100 (2015), 191-250.

[8] Beauville, A., Variétés de Prym et jacobiennes intermédiaires. Ann. Sci. École Norm. Sup., 10 (1977), 309-391.

[9] - Variétés Kähleriennes dont la première classe de Chern est nulle. J. Differential Geom., 18 (1983), 755-782.

[10] — Le groupe de monodromie des familles universelles d'hypersurfaces et d'intersections complètes, in Complex Analysis and Algebraic Geometry (Göttingen, 1985), Lecture Notes in Math., 1194, pp. 8-18. Springer, Berlin-Heidelberg, 1986.

[11] — Determinantal hypersurfaces. Michigan Math. J., 48 (2000), 39-64.

[12] - Vector bundles on the cubic threefold, in Symposium in Honor of C. H. Clemens (Salt Lake City, UT, 2000), Contemp. Math., 312, pp. 71-86. Amer. Math. Soc., Providence, RI, 2002.

[13] Beauville, A. \& Donagi, R., La variété des droites d'une hypersurface cubique de dimension 4. C. R. Acad. Sci. Paris Sér. I Math., 301 (1985), 703-706. 
[14] Bloch, S. \& SRinivas, V., Remarks on correspondences and algebraic cycles. Amer. J. Math., 105 (1983), 1235-1253.

[15] Casalaina-Martin, S., Grushevsky, S., Hulek, K. \& Laza, R., Extending the Prym map to toroidal compactifications of the moduli space of abelian varieties. J. Eur. Math. Soc. (JEMS), 19 (2017), 659-723.

[16] - Complete moduli of cubic threefolds and their intermediate Jacobians. Preprint, 2015. arXiv: 1510.08891 [math.AG].

[17] Casalaina-Martin, S. \& Laza, R., The moduli space of cubic threefolds via degenerations of the intermediate Jacobian. J. Reine Angew. Math., 633 (2009), 29-65.

[18] - Simultaneous semi-stable reduction for curves with ADE singularities. Trans. Amer. Math. Soc., 365 (2013), 2271-2295.

[19] Clemens, C. H. \& Griffiths, P. A., The intermediate Jacobian of the cubic threefold. Ann. of Math., 95 (1972), 281-356.

[20] Cook, P. R., Compactified Jacobians and curves with simple singularities, in Algebraic Geometry (Catania, 1993/Barcelona, 1994), Lecture Notes in Pure and Appl. Math., 200, pp. 37-47. Dekker, New York, 1998.

[21] Deligne, P., Théorie de Hodge. II. Inst. Hautes Études Sci. Publ. Math., 40 (1971), 5-57.

[22] Donagi, R. \& Markman, E., Spectral covers, algebraically completely integrable, Hamiltonian systems, and moduli of bundles, in Integrable Systems and Quantum Groups (Montecatini Terme, 1993), Lecture Notes in Math., 1620, pp. 1-119. Springer, BerlinHeidelberg, 1996.

[23] Fantechi, B., Göttsche, L. \& van Straten, D., Euler number of the compactified Jacobian and multiplicity of rational curves. J. Algebraic Geom., 8 (1999), 115-133.

[24] Friedman, R. \& Smith, R., Degenerations of Prym varieties and intersections of three quadrics. Invent. Math., 85 (1986), 615-635.

[25] Fulton, W., Intersection Theory. Ergebnisse der Mathematik und ihrer Grenzgebiete, 2. Springer, Berlin-Heidelberg, 1998.

[26] Green, M. \& Lazarsfeld, R., Special divisors on curves on a K3 surface. Invent. Math., 89 (1987), 357-370.

[27] Griffiths, P.A., On the periods of certain rational integrals. I, II. Ann. of Math., 90 (1969), 460-495, 496-541.

[28] Gritsenko, V., Hulek, K. \& Sankaran, G. K., Moduli spaces of polarized symplectic O'Grady varieties and Borcherds products. J. Differential Geom., 88 (2011), 61-85.

[29] Grothendieck, A., Él'ements de géométrie algébrique. II. Étude globale élémentaire de quelques classes de morphismes. Inst. Hautes Études Sci. Publ. Math., 8 (1961), 222 pp.

[30] Harris, J., Roth, M. \& Starr, J., Curves of small degree on cubic threefolds. Rocky Mountain J. Math., 35 (2005), 761-817.

[31] Hartshorne, R., Algebraic Geometry. Graduate Texts in Mathematics, 52. Springer, New York-Heidelberg, 1977.

[32] Hassett, B., Special cubic fourfolds. Compositio Math., 120 (2000), 1-23.

[33] Huybrechts, D., Compact hyper-Kähler manifolds: basic results. Invent. Math., 135 (1999), 63-113.

[34] Huybrechts, D. \& Lehn, M., The Geometry of Moduli Spaces of Sheaves. Cambridge Mathematical Library. Cambridge University Press, Cambridge, 2010.

[35] Hwang, J.-M. \& NAGAI, Y., Algebraic complete integrability of an integrable system of Beauville. Ann. Inst. Fourier (Grenoble), 58 (2008), 559-570.

[36] Iliev, A. \& Manivel, L., Cubic hypersurfaces and integrable systems. Amer. J. Math., 130 (2008), 1445-1475. 
[37] Iliev, A. \& Markushevich, D., The Abel-Jacobi map for a cubic threefold and periods of Fano threefolds of degree 14. Doc. Math., 5 (2000), 23-47.

[38] Kaledin, D., Lehn, M. \& Sorger, C., Singular symplectic moduli spaces. Invent. Math., 164 (2006), 591-614.

[39] Kollár, J., Laza, R., Saccà, G. \& Voisin, C., Remarks on degenerations of hyperKähler manifolds. Preprint, 2017. arXiv:1704.02731 [math. AG].

[40] Kuznetsov, A. \& Markushevich, D., Symplectic structures on moduli spaces of sheaves via the Atiyah class. J. Geom. Phys., 59 (2009), 843-860.

[41] Kuznetsov, A. G., Derived category of a cubic threefold and the variety $V_{14}$. Tr. Mat. Inst. Steklova, 246 (2004), 183-207 (Russian); English translation in Proc. Steklov Inst. Math., 246 (2004), 171-194.

[42] Lehn, C., Sorger, C. \& van Straten, D., Twisted cubics on cubic fourfolds. To appear in J. Reine Angew. Math.

[43] Markushevich, D., Integrable systems from intermediate Jacobians of 5-folds. Mat. Contemp., 41 (2012), 49-60.

[44] Markushevich, D. \& Tikhomirov, A.S., The Abel-Jacobi map of a moduli component of vector bundles on the cubic threefold. J. Algebraic Geom., 10 (2001), 37-62.

[45] - New symplectic $V$-manifolds of dimension four via the relative compactified Prymian. Internat. J. Math., 18 (2007), 1187-1224.

[46] Markushevich, D. G. \& Tikhomirov, A. S., Symplectic structure on a moduli space of sheaves on a cubic fourfold. Izv. Ross. Akad. Nauk Ser. Mat., 67 (2003), 131-158.

[47] Mukai, S., Symplectic structure of the moduli space of sheaves on an abelian or K3 surface. Invent. Math., 77 (1984), 101-116.

[48] Mumford, D., Rational equivalence of 0-cycles on surfaces. J. Math. Kyoto Univ., 9 (1968), 195-204.

[49] — Prym varieties. I. (1974), 325-350.

[50] O'Grady, K. G., Desingularized moduli spaces of sheaves on a K3. J. Reine Angew. Math., 512 (1999), 49-117.

[51] - A new six-dimensional irreducible symplectic variety. J. Algebraic Geom., 12 (2003), 435-505.

[52] Du Plessis, A. A. \& Wall, C. T. C., Versal deformations in spaces of polynomials of fixed weight. Compositio Math., 114 (1998), 113-124.

[53] - Hypersurfaces in $\mathbf{P}^{n}$ with 1-parameter symmetry groups. II. Manuscripta Math., 131 (2010), 111-143.

[54] Ran, Z., Deformations of maps, in Algebraic Curves and Projective Geometry (Trento, 1988), Lecture Notes in Math., 1389, pp. 246-253. Springer, Berlin-Heidelberg, 1989.

[55] Rapagnetta, A., On the Beauville form of the known irreducible symplectic varieties. Math. Ann., 340 (2008), 77-95.

[56] Rego, C. J., The compactified Jacobian. Ann. Sci. École Norm. Sup., 13 (1980), 211-223.

[57] Rim, D. S., Equivariant G-structure on versal deformations. Trans. Amer. Math. Soc., 257 (1980), 217-226.

[58] SaccÀ, G., Fibrations in abelian varieties associated to Enriques surfaces. Ph.D. Thesis, Princeton University, Princeton, NJ, 2013.

[59] — Relative compactified Jacobians of linear systems on Enriques surfaces. Preprint, 2013.

[60] Sernesi, E., Deformations of Algebraic Schemes. Grundlehren der Mathematischen Wissenschaften, 334. Springer, Berlin-Heidelberg, 2006.

[61] Shustin, E. I., Versal deformations in a space of plane curves of fixed degree. Funktsional. Anal. i Prilozhen., 21 (1987), 90-91 (Russian); English translation in Functional Anal. Appl., 21 (1987), 82-84. 
[62] Shustin, E. I. \& Tyomkin, I., Versal deformation of algebraic hypersurfaces with isolated singularities. Math. Ann., 313 (1999), 297-314.

[63] Tuurin, A. N., Five lectures on three-dimensional varieties. Uspehi Mat. Nauk, 27 (1972), 3-50 (Russian); English translation in Russian Math. Surveys, 27 (1972), 1-53.

[64] Voisin, C., Intrinsic pseudo-volume forms and $K$-correspondences, in The Fano Conference, pp. 761-792. Univ. Torino, Turin, 2004.

[65] — Hodge Theory and Complex Algebraic Geometry. I, II. Cambridge Studies in Advanced Mathematics, 76, 77. Cambridge University Press, Cambridge, 2007.

[66] - Chow Rings, Decomposition of the Diagonal, and the Topology of Families. Annals of Mathematics Studies, 187. Princeton University Press, Princeton, NJ, 2014.

[67] Yoshioka, K., Moduli spaces of twisted sheaves on a projective variety, in Moduli Spaces and Arithmetic Geometry, Adv. Stud. Pure Math., 45, pp. 1-30. Math. Soc. Japan, Tokyo, 2006.

RADU LAZA

Stony Brook University

100 Nicolls Rd.

Stony Brook, NY 11794

U.S.A.

radu.laza@stonybrook.edu

Clatre Voisin

Collège de France

3 rue d'Ulm

FR-75005 Paris

France

claire.voisin@imj-prg.fr

Received February 23, 2016

Received in revised form October 31, 2016
Giulia SaCCÀ

Stony Brook University

100 Nicolls Rd.

Stony Brook, NY 11794

U.S.A.

giulia.sacca@stonybrook.edu 